\title{
MAMMALIAN HIBERNATION: GENE EXPRESSION AND TRANSCRIPTION FACTOR REGULATION OF THE UNFOLDED PROTEIN RESPONSE, APOPTOSIS, AND ATROPHY IN GROUND SQUIRRELS
}

\author{
By \\ Mamady Hapsatou \\ B.Sc. Université du Québec a Montréal, 1995 \\ M.Sc. Université de Sherbrooke, 1999
}

A Thesis Submitted to the Faculty of Graduate Studies and Research in partial fulfillment of the requirements for the degree of

\author{
Doctor of Philosophy \\ Department of Biology
}

Carleton University

Ottawa, Ontario, Canada

(C) copyright 2006

Mamady Hapsatou 


$\begin{array}{ll}\begin{array}{l}\text { Library and } \\ \text { Archives Canada }\end{array} & \begin{array}{l}\text { Bibliothèque et } \\ \text { Archives Canada }\end{array} \\ \begin{array}{l}\text { Published Heritage } \\ \text { Branch }\end{array} & \begin{array}{l}\text { Direction du } \\ \text { Patrimoine de l'édition }\end{array} \\ \begin{array}{l}\text { 395 Wellington Street } \\ \text { Ottawa ON K1A ON4 }\end{array} & \begin{array}{l}\text { 395, rue Wellington } \\ \text { Ottawa ON K1A ON4 } \\ \text { Canada }\end{array}\end{array}$

Your file Votre référence ISBN: 978-0-494-18222-2 Our file Notre référence ISBN: 978-0-494-18222-2

NOTICE:

The author has granted a nonexclusive license allowing Library and Archives Canada to reproduce, publish, archive, preserve, conserve, communicate to the public by telecommunication or on the Internet, loan, distribute and sell theses worldwide, for commercial or noncommercial purposes, in microform, paper, electronic and/or any other formats.

The author retains copyright ownership and moral rights in this thesis. Neither the thesis nor substantial extracts from it may be printed or otherwise reproduced without the author's permission.
AVIS:

L'auteur a accordé une licence non exclusive permettant à la Bibliothèque et Archives Canada de reproduire, publier, archiver, sauvegarder, conserver, transmettre au public par télécommunication ou par l'Internet, prêter, distribuer et vendre des thèses partout dans le monde, à des fins commerciales ou autres, sur support microforme, papier, électronique et/ou autres formats.

L'auteur conserve la propriété du droit d'auteur et des droits moraux qui protège cette thèse. $\mathrm{Ni}$ la thèse ni des extraits substantiels de celle-ci ne doivent être imprimés ou autrement reproduits sans son autorisation.
In compliance with the Canadian

Privacy Act some supporting forms may have been removed from this thesis.

While these forms may be included in the document page count, their removal does not represent any loss of content from the thesis.
Conformément à la loi canadienne sur la protection de la vie privée, quelques formulaires secondaires ont été enlevés de cette thèse.

Bien que ces formulaires aient inclus dans la pagination, il n'y aura aucun contenu manquant.

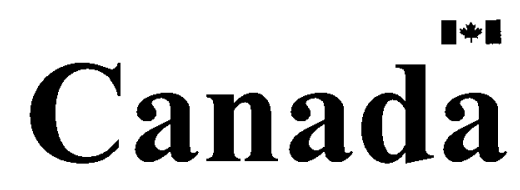




\begin{abstract}
Various mammalian species hibernate as a way to survive extended winter periods of food scarcity and cold environmental conditions. Hibernation is an energy-conserving strategy, characterized by periods of torpor with extreme decreases in core body temperature and strong metabolic rate depression, interrupted by brief periods of arousal to euthermia. To endure the conditions of cold torpor, as well as the wide fluctuations over cycles of torpor-arousal, differential expression of genes and their tight regulation is required. The present studies examined changes in the expression and regulation of selected genes involved in the unfolded protein response, muscle atrophy and antiapoptotic defense during hibernation in thirteen-lined ground squirrels, Spermophilus tridecemlineatus. Despite overall suppression of transcription and translation during torpor, selected genes and their products were up-regulated. The molecular chaperone GRP78 increased in BAT and brain of torpid animals, indicating endoplasmic reticulum stress and a role for GRP78 in alleviating stresses that cause protein misfolding during hibernation. Regulation of the grp78 gene by the activating transcription factor ATF4 via the PERK/eIF2 $\alpha /$ ATF4 pathway was shown to be important in hibernation; ATF4 protein expression increased in BAT, brain and skeletal muscle of hibernating squirrels and ATF4 DNA-binding activity increased in hibernating brain. Subcellular localization of ATF4 showed that this transcription factor and its cofactor, pCREB-1, were translocated into the nucleus during hibernation where they could activate downstream genes. Another transcription factor, FoxOla, and the downstream genes that it controls via the PI3K/AKT/FOXO pathway can induce muscle atrophy. Hibernators appear to counteract this by phosphorylating and inactivating FoxO1a in heart and skeletal muscle and
\end{abstract}


strongly suppressing FoxO1a DNA binding activity by $76 \%$ in muscle during torpor. Finally, the anti-apoptotic proteins, Bcl-XL and Bcl-2, showed enhanced expression in tissues of ground squirrels whereas the pro-apoptotic protein, BAD, was suppressed via phosphorylation during torpor. These results show that anti-apoptotic defense is also important to cell survival in hibernation. The data in this thesis enhance our knowledge of the molecular mechanisms that govern hibernation and the role played by selected transcription factors in regulating subsets of genes that are physiologically relevant to the hibernation phenotype. 


\section{Acknowledgements}

First and foremost, I would like to thank my thesis supervisor Dr. Kenneth B. Storey who accepted me into his Lab and gave me a chance to develop my skills and learn, in all aspects, what the real "SCIENCE" is. Dr. Storey's teaching, guidance, and support have provided me with a solid foundation and I have become more confident scientist. Thanks for giving me that role of "the mother of the lab". Thanks to Jan Storey, for her perfect editing, insightful suggestions and her encouragement with my writing. I am extremely grateful.

I would also like to thank the members of my committee, Dr. Bill Willmore and Dr. Tom Moon, for their support and valuable suggestions. Many thanks also go to all my friends and lab colleagues Jiayun, Ashley and Pier, Khalil, Chris, Jacques, Jeremy and all former and current Storey's lab members, for their help, support, interest and valuable scientific discussions.

Finally, I would like to give a special thank to my lovely husband Mamat and my little ones Abba and Attou for their support and encouragement. Thanks to my parents for their firmest support from thousands of miles away, through long distance calls. 


\section{Table of Contents}

Title Page

Acceptance Sheet

$\begin{array}{ll}\text { Abstract } & \text { iii }\end{array}$

Acknowledgements $\quad$ v

Table of Contents vi

List of Abbreviations vii

List of Figures $\quad$ X

Chapter 1 General Introduction 1

$\begin{array}{lll}\text { Chapter } 2 & \text { Up-regulation of the endoplasmic reticulum molecular } & 18\end{array}$ chaperone GRP78 during hibernation in thirteen-lined ground squirrels

Chapter 3 Coping with the stress: expression of ATF4, ATF6 and downstream targets in hibernating ground squirrel tissues

Chapter 4 Muscle disuse atrophy: profile and assessment of FoxO1a activation, MAFbx/atrogin-1, Bcl-3 and NFkB p50 in skeletal muscle and heart during hibernation

Chapter 5 Bcl-2 family members and anti-apoptotic defense in hibernating ground squirrels

Chapter 6 General Discussion

Publication List

References 


\section{List of Abbreviations}

\begin{tabular}{|c|c|}
\hline $\mathrm{AA}$ & amino acid \\
\hline ADP & adenosine diphosphate \\
\hline AKT & protein kinase B \\
\hline ATF & activating transcription factor \\
\hline ATP & adenosine triphosphate \\
\hline Bad & Bcl-2-associated death protein \\
\hline BAT & brown adipose tissue \\
\hline $\mathrm{Bcl}-2$ & B-cell leukemia/lymphoma 2 \\
\hline Bcl-xL & B cell leukemia/lymphoma xL \\
\hline BLAST & Basic Local Alignment Search Tool \\
\hline cDNA & complementary deoxyribonucleic acid \\
\hline CDS & coding sequence \\
\hline CHOP & CCAAT/enhance-binding protein $(\mathrm{C} / \mathrm{EBP})$ homologous protein \\
\hline CRE & cAMP response element \\
\hline CREB & cAMP response element binding protein \\
\hline DEPC & diethylpyrocarbonate \\
\hline DTT & dithiolthreitol \\
\hline ETC & electron transport chain \\
\hline EDTA & ethylenediamine tetraacetic acid \\
\hline $\mathrm{eIF} 2 \alpha$ & eukaryotic initiation factor $2 \alpha$ subunit \\
\hline EMSA & Electrophoretic Mobility Shift Assay \\
\hline ER & endoplasmic reticulum \\
\hline
\end{tabular}




\begin{tabular}{|c|c|}
\hline ERAD & ER associated degradation \\
\hline FABP & fatty acid binding protein \\
\hline FOXO & Forkhead box, class O proteins \\
\hline GADD & growth arrest and DNA damage gene \\
\hline GRP78 & glucose regulated protein 78 \\
\hline HIF-1 & hypoxia-inducible factor-1 \\
\hline Hsp. & heat shock protein \\
\hline I $\kappa B$ & inhibitor of NFKB \\
\hline IRE1 & Inositol requiring kinase 1 \\
\hline $\mathrm{kb}$ & kilobase \\
\hline $\mathrm{kDa}$ & kilodalton \\
\hline MAFbx & Muscle atrophy $\mathrm{F}$ box \\
\hline MAPK & mitogen-activated protein kinase \\
\hline MLC1v & ventricular isoform of myosin light chain 1 \\
\hline MOPS & 2-(N- morpholine) proanesulfonic acid \\
\hline mRNA & messenger RNA \\
\hline MuRF1 & Muscle ring finger protein 1 \\
\hline $\mathrm{NF} \kappa \mathrm{B}$ & Nuclear factor kappa B \\
\hline NPY & neuropeptide $Y$ \\
\hline Nrf2 & nuclear factor-erythroid 2 related factor -2 \\
\hline ORF & open reading frame \\
\hline PAGE & polyacrylamide gel electrophoresis \\
\hline PCR & polymerase chain reaction \\
\hline
\end{tabular}




\begin{tabular}{|c|c|}
\hline PDK & pyruvate dehydrogenase kinase \\
\hline PERK & PKR-like endoplasmic reticulum kinase \\
\hline PGC & PPAR $\gamma$ co-activator \\
\hline PMSF & phenylmethanesulfonyl fluoride \\
\hline PUFA & polyunsaturated fatty acid \\
\hline PVDF & polyvinylidine fluoride \\
\hline RACE & rapid amplification of cDNA ends \\
\hline ROS & reactive oxygen species \\
\hline rRNA & ribosomal RNA \\
\hline RT & room temperature $\left(\sim 21^{\circ} \mathrm{C}\right)$ \\
\hline SDS & sodium dodecyl sulfate \\
\hline $\mathrm{Tb}$ & body temperature \\
\hline TEMED & $\mathrm{N}, \mathrm{N}, \mathrm{N}^{\prime}, \mathrm{N}^{\prime}$-tetramethylethylenediamine \\
\hline Tris & tris (hydroxymethyl) aminomethane \\
\hline UCP & uncoupling protein \\
\hline UPR & unfolded protein response \\
\hline XBP1 & $\mathrm{X}$-box binding protein \\
\hline WAT & white adipose tissue \\
\hline
\end{tabular}




\section{List of figures}

Fig. 1.1 Body temperature (Tb) of a golden-mantled ground squirrel as a function of time over the winter hibernation season.

Fig. 1.2 Distribution map for thirteen-lined ground squirrels, $S$. tridecemlineatus.

Fig. 2.1 Model of GRP78 role: central regulator for ER stress signaling and survival.

Fig. 2.2 Effect of hibernation on grp 78 mRNA levels in seven tissues of thirteen-lined ground squirrels.

Fig. 2.3 Complete nucleotide and deduced amino acid sequence of $g r p 78$ from $S$. tridecemlineatus.

Fig. 2.4 Alignment of $S$. tridecemlineatus GRP78 full length amino acid sequence with human, mouse and rat sequences.

Fig. 2.5 Cladogram and homology matrix showing the percent identities between the protein sequences of GRP78 from ground squirrel, human, mouse and rat.

Fig. 2.6 Representative 2D-PAGE Western blot of muscle extract from euthermic thirteen-lined ground squirrel immunoblotted with antiGRP78 antibody.

Fig. 2.7 GRP78 protein expression in seven tissues from euthermic (E) and hibernating $(\mathrm{H})$ ground squirrels.

Fig. 2.8 Electrophoretic mobility shift and supershift assays using ATF4 DNA binding domain from grp78 promoter as probe and nuclear extracts from brain of $S$. tridecemlineatus.

Fig. 3.1 Model of UPR signaling in mammalian cells, depicting role of ATF4 during ER stress.

Fig. 3.2 p-PERK protein expression in brain and protein levels of ATF6 in three tissues of euthermic and hibernating ground squirrels.

Fig. 3.3 Representative Western blots showing ATF4 protein levels in six tissues from euthermic $(\mathrm{E})$ and hibernating $(\mathrm{H})$ ground squirrels. 
Fig. 3.4 Partial nucleotide and deduced amino acid sequences of ATF4 from $S$. tridecemlineatus.

Fig. 3.5 Alignment of $S$. tridecemlineatus ATF4 partial amino acid 74 sequence with human, mouse and rat sequences

Fig. 3.6 Cladogram and homology matrix showing the percent identities between the protein sequences of ATF4 from ground squirrel, human, mouse and rat.

Fig. 3.7 Effect of hibernation on atf 4 mRNA levels in six tissues of 76 thirteen-lined ground squirrels.

Fig. 3.8 Western blot analysis of total CREB-1 protein and phosphorylated CREB-1 protein expression in tissues from euthermic and hibernating ground squirrels.

Fig. 3.9 Analysis of ATF6 (50 $\mathrm{kDa})$ protein distribution between cytoplasmic and nuclear fractions in three tissues of euthermic $(\mathrm{E})$ and hibernating $(\mathrm{H})$ ground squirrels.

Fig. 3.10 Distribution of the transcription factors ATF4 (A) and p-CREB1 (B) between cytoplasmic and nuclear fractions of brain and muscle from euthermic and hibernating ground squirrels.

Fig. 3.11 Representative Western blots of GADD153 (CHOP) (A) and GADD34 (B) protein levels in ground squirrel tissues.

Fig. 4.1 Analysis of FoxOla transcript levels in ground squirrel heart and 101 skeletal muscle.

Fig. 4.2 FoxO1a and pFoxO1a Ser 256 protein expression in muscle and 102 heart of thirteen-lined ground squirrels.

Fig. 4.3 Distribution of FoxO1a and pFoxOla between cytoplasmic and 103 nuclear fractions in skeletal muscle of $S$. tridecemlineatus.

Fig. 4.4 FoxO1a DNA binding activity in heart and skeletal muscle of 104 euthermic and hibernating thirteen-lined ground squirrels.

Fig. 4.5 MAFbx / atrogin-1 protein expression in heart and muscle of 105 thirteen-lined ground squirrels.

Fig. 4.6 Bcl-3 protein expression in heart and muscle of thirteen-lined 106 ground squirrels. 
Fig. 4.7 NFאB p50 protein expression in heart and muscle of thirteenlined ground squirrels.

Fig. 4.8 Analysis of NFKB p50 protein distribution between cytoplasmic and nuclear fractions in heart and skeletal muscle of euthermic (E) and hibernating $(\mathrm{H})$ ground squirrels.

Fig. 4.9 Analysis of Bcl-3 protein distribution between cytoplasmic and 109 nuclear fractions in heart of euthermic $(\mathrm{E})$ and hibernating $(\mathrm{H})$ ground squirrels.

Fig. 5.1 Partially amplified $b c l-x l$ cDNA sequence and deduced amino acid sequence of Bcl-XL from brain of thirteen-lined ground squirrels.

Fig. 5.2

Alignment of $S$. tridecemlineatus Bcl-XL partial amino acid sequence with human, mouse and rat sequences

Fig. 5.3 Homology tree and cladogram showing the percent identities between the nucleotide and amino acid sequences of $b c l-x l$ from ground squirrel aligned with human, mouse and rat.

Fig. 5.4 Analysis of $b c l-x l$ transcript levels in ground squirrel tissues during hibernation.

Fig. 5.5 Bcl-XL protein expression in six tissues of thirteen-lined ground squirrels.

Fig. 5.6 Bcl-2 protein expression in six tissues of thirteen-lined ground squirrels.

Fig. 5.7 BAD protein expression in heart and muscle of thirteen-lined ground squirrels.

Fig. 5.8 Protein expression of three phosphorylated forms of BAD in heart and muscle of thirteen-lined ground squirrels.

Fig. 6.1 Analysis of grp78 expression in brown adipose tissue and brain of the little brown bat Myotis lucifugus.

Fig. 6.2 A model for FoxO activation through the AKT signaling network during atrophy and hypertrophy. 


\section{Chapter 1}

\section{General Introduction}




\section{INTRODUCTION}

Many small mammals in north-temperate regions solve the problems of winter scarcity of food and low environmental temperatures by entering a prolonged and controlled state of dormancy, known as hibernation. The hibernation season typically consists of long periods of cold torpor (each may be 3 weeks or more) where body temperature ( $\mathrm{Tb}$ ) drops to near-ambient (often close to $0^{\circ} \mathrm{C}$ ) interspersed with brief $(<20$ hours) periods of arousal to euthermia ( $\mathrm{Tb} \sim 37^{\circ} \mathrm{C}$ ) (Lyman et al., 1982). Thus, hibernation is an energy conserving strategy involving physiological and behavioral accommodations to low metabolic rate and low body temperature for extended periods (French, 1988). According to Wang (1989), hibernating ground squirrels can conserve up to $88 \%$ of the energy that would otherwise be required to maintain a euthermic body temperature over the winter. Euthermy is a thermoregulatory state where heat is generated to maintain body temperature relatively high and constant (French, 1988). Therefore, small endotherms must sacrifice a large amount of their energy supply to compensate for heat loss during cold exposure. The adaptive value of the hibernating phenotype is then realized by eliminating the need to maintain a constant, high $\mathrm{Tb}$ by entering torpor and allowing $\mathrm{Tb}$ to fall to near ambient. Thus, there is growing appreciation of the diversity of torpor patterns with regard to the minimum $\mathrm{Tb}$ values reached during torpor and the duration of torpor bouts (Dausmann et al., 2000; Geiser and Ruf, 1995; Carey et al., 2003a).

\section{Preparation for hibernation}

Prior to entering hibernation, fat-storing hibernators, such as ground squirrels, get ready for winter by eating extra food in the late summer and early autumn (hyperphagia) 
and storing it as body fat. They select an appropriate hibernaculum such as underground burrows, caves or mines that provide shelter from potential predators, temperature extremes and potential desiccation. In fact, most hibernacula show temperatures a few degrees above $0^{\circ} \mathrm{C}$ even when outside air temperature minima are below freezing (Geiser, 1988). By contrast, other hibernators, such as chipmunks and pocket mice lay in stores of food in their burrow, to eat later in the winter. They store 25 to 50 times the amount of energy in the form of seeds and display no significant increases in autumn body mass compared to fat-storing species that practically double their body mass (French, 1988; Geiser, 1988).

Entry into full deep torpor is a gradual process (Fig. 1.1). Early in the hibernation season, the animal goes through a series of "test-drops" while sleeping during which its Tb drops a few degrees and then returns to normal. Gradually, the animal cools to lower temperatures (typically within a degree or less of the ambient temperature) and torpor bouts become longer. One hypothesis that has prevailed in the hibernation literature is that hibernating animals have some kind of opiate, chemically related to morphine, in their blood which induces torpor. Wang and Lee (1996) suggested that as the days get shorter, temperature falls, and food becomes scarce, this Hibernation-Inducing Trigger (HIT) is produced and stimulates entry into torpor. However, although some studies have shown that plasma from a hibernating animal can induce torpor in a nonhibernating individual, the chemical responsible has still not been isolated.

\section{Torpor state}

The length of the torpid episodes increases during midwinter and then shortens again toward spring. It has been suggested that, on average, small fat-storing species such 
as bats (i.e. Myotis lucifugus) and ground squirrels (i.e. Spermophilus lateralis) display longer torpor bouts than large species such as marmots (i.e. Marmota marmota) and generally save more energy (Geiser and Ruf, 1995). During torpor, all physiological functions are suppressed to low levels. Hibernating ground squirrels, for example, show a heart rate of only 5-10 beats per minute compared with the euthermic value of 350-400 beats per minute (Storey, 2003). Respiration is reduced from 100-200 breaths/min to 4-6 breaths/min and some species show long apnoic periods (McArthur and Milsom, 1991). Renal function is greatly reduced or ceases altogether (Zatzman 1984; Carey et al, 2003a). By entering a dormant state, hibernators minimize their consumption of metabolic fuels and can survive for many months by slowly oxidizing stored body fuel reserves, mainly lipids. Fat-storing hibernators rely on the products of lipid hydrolysis (fatty acids and glycerol) obtained from white adipose tissue (WAT) as their primary fuel source. The low metabolic rate of the torpid state lowers the need for food intake, such that the reserves of fat that were laid down prior to the onset of hibernation are sufficient to carry the animal through the winter. By contrast, food-storing hibernators such as chipmunks (i.e. Tamia sibericus) and hamsters (i.e. Mesocricetus auratus) use cached food that is ingested during periodic arousals (Humphries et al., 2003). The composition of body lipid depots is also important for successful hibernation. During the prehibernation fattening period, fat-storing animals increase their intake of foods containing polyunsaturated fatty acids (PUFAs) and elevate the percentage of PUFAs in their lipid depots. High PUFA content maintains the fluidity of the lipid depots so that they can be metabolized at near $0^{\circ} \mathrm{C} \mathrm{Tb}$. 


\section{Arousal from torpor}

Torpor is interrupted by periods of arousal where the animal returns briefly to the euthermic state. The intense metabolic activity that is required to reheat the animal actually consumes most of the winter energy budget. During these interbout arousals, $\mathrm{Tb}$ rises to $36-38^{\circ} \mathrm{C}$ and is maintained for several hours before reentry into torpor begins (Carey et al, 2003a). Physiological functions are restored rapidly to near-normal levels. The pattern of arousal varies between hibernating species and can last for one or two hours in small hibernators (e.g. bats) up to about a day in large marmots. Moreover, the frequency of arousal is also affected by body size, being less frequent in the smaller hibernators than it is in larger ones (French, 1988). Why these arousals occur is not yet clear. However, numerous hypotheses have been proposed about periodic arousals. According to Humphries et al. (2003), the function of arousals involves recovery from physiological costs accumulated during metabolic depression, including oxidative stress, reduced immunocompetence, and neuronal tissue damage. Geiser and Ruf (1995) postulated that physiological imbalances that develop during hibernation are rectified during normothermic periods. This could include depletion of nutrients (glucose to be resynthesized) or loss of body water (that has to be replenished) or accumulation of noxious substances (that cannot be excreted because of the reduced blood pressure during torpor). In addition, it has been reported that intensive protein synthesis occurs during the interbout periods in tissues of ground squirrels including heart, liver, spleen, pancreas, and kidney (Zhegunov et al, 1988; Carey et al, 2003a). Thomas and Cloutier (1992) followed by Thomas and Geiser (1997) demonstrated that evaporation during prolonged torpor bouts leads to dehydration, forcing hibernators to arouse to replenish depleted 
water stores. Because evaporation is temperature dependent, a water-balance theory also predicts a correlation between $\mathrm{Tb}$ and arousal frequency. Therefore, they have concluded that the rate of evaporative water loss predicts torpor bout duration and hence arousal frequency.

Although many hypotheses have been proposed for the importance and functions of periodic arousals, the signal(s) that initiate arousal from torpor is still not well understood. It has been suggested that a change in the $\mathrm{Tb}$ setpoint near the end of a torpor bout plays a role in initiating the thermoregulatory responses that lead to increased metabolic rate and a rapid return of $\mathrm{Tb}$ to euthermic levels (Carey et al., 2003a). According to Geiser and Kenagy (1988), the thermal dependence of torpor bout duration of hibernators supports the view that periodic arousals are determined by both metabolic processes and the body temperature experienced during torpor.

During arousal, the $\mathrm{Tb}$ rises exponentially. Initial heating is due to nonshivering thermogenesis but this is aided by skeletal muscle shivering that begins as Tb rises above $20^{\circ} \mathrm{C}$ until the animal reaches normothermy. Geiser (1996) reported that the rewarming rates are mass-dependent. Small species such as bats can rewarm at a rate of over $1^{\circ} \mathrm{C}$ per minute, while large species (marmots) manage around $0.1^{\circ} \mathrm{C}$ per minute. As spring approaches, the period of torpor decreases and the period of arousal increases until one such arousal defines the end of hibernation (Geiser, 1996).

It is known that to survive the cold winter, small mammals strongly suppress their metabolic rate, fall into deep torpor and decrease their $\mathrm{Tb}$. As $\mathrm{Tb}$ falls from the euthermic level, the rate of all metabolic processes also declines exponentially giving rise to the $\mathrm{Q}_{10}$ effect. $\mathrm{A} 10^{\circ} \mathrm{C}$ drop in $\mathrm{Tb}$ decreases metabolic rate by about $50 \%\left(\mathrm{Q}_{10}=2\right)$, which is 
observable as a decline in oxygen consumption or $\mathrm{CO}_{2}$ production (Speakman and Thomas, 2003). Indeed, Frerichs et al. (1995) have reported the reduction of metabolic rate in hibernating ground squirrels to lower levels as compared to the euthermic animals, corresponding to Q10 value of 4.3. Hence the importance of energy savings during hibernation is demonstrated.

\section{Model hibernator}

The thirteen-lined ground squirrel, Spermophilus tridecemlineatus, is a small prairie mammal with body length of $\sim 24 \mathrm{~cm}$. Adults weigh $130-180 \mathrm{~g}$ but they gain considerable weight (up to $4 \mathrm{~g}$ fat per day) in late summer to raise their body mass to 220 $240 \mathrm{~g}$ prior to hibernation. According to Cleary and Craven (1994), the range of thirteenlined ground squirrels extends from Central Alberta, Manitoba, and Saskatchewan in the North to Texas and New Mexico in the South, and from Central Ohio in the east to Colorado in the west (Fig. 1.2). They feed primarily on seeds and also eat many insects, including grasshoppers and cutworms. The species is an obligate hibernator and squirrels remain in their underground burrows continuously throughout the winter, entering hibernation in September or early October and emerging between late March and early May in the Northern parts of their range.

This thesis focuses more on the responses to hibernation by several organs of 13 lined ground squirrels, primarily focusing on brown adipose tissue (BAT), brain, heart, and skeletal muscle but with some analysis of kidney, liver and lung as well. 


\section{Brown adipose tissue}

Hibernators have two kinds of adipose tissue: regular WAT and the specialized thermogenic BAT. The cells of BAT are packed with small fat droplets and many large mitochondria. However, unlike normal mitochondria that couple the energy released from substrate oxidation into the synthesis of ATP, in BAT mitochondria the flow of electrons down the electron transport chain (ETC) is uncoupled from oxidative phosphorylation so that energy is released as heat. This form of heat production is called nonshivering thermogenesis as compared to the heat produced by shivering in muscle which results from high rates of ATP hydrolysis during muscular contraction (Neuweiler, 2000). Uncoupling is achieved by the presence of uncoupling protein 1 (UPC-1) in the inner mitochondrial membrane; this protein allows protons that are pumped out of the matrix by the ETC and into the intermembrane space to re-enter the matrix without passing through the $\mathrm{F}_{0} \mathrm{~F}_{1}$-ATPase. BAT is responsible for the generation of the large amount of heat that is needed to rewarm the animal during arousals from torpor (Buck and Barnes, 2000). BAT also provides low level heat generation when ambient environmental temperature drops below $0^{\circ} \mathrm{C}$ and the animal must prevent its tissues from freezing.

\section{Brain}

Despite dramatically reduced electrical and metabolic activity, significant aspects of the central nervous system function must continue during torpor (Kilduff et al., 1990). Hibernating mammals remain fully capable of sensing external stimuli such as ambient temperature change, and maintain a respiratory and cardiac rhythm during torpor; hence, this involves neurohumoral cues (Carey et al., 2003a). Through the torpor-arousal cycles 
of hibernation, small hibernators experience a pronounced fluctuation in cerebral blood flow but no brain damage occurs. Numerous neuroprotective aspects may contribute to this natural tolerance to cerebral ischemia (Zhou et al., 2001). Several immediate-early genes and including transcription factors, c-fos, c-jun, and junB, are differentially expressed in the brain across the hibernation cycle (O'Hara et al., 1999), likely reflecting a link between neuronal activation and immediate-early gene expression. Recent studies by El Ouezzani et al. (2001) on hibernating jerboas (Jaculus orientalis) have shown an increase in the expression of mRNA encoding neuropeptide Y (NPY), a transmitter in the nervous system, involved in metabolic regulation. Stimulation of the NPY gene within neurons of the arcuate nucleus (ARC) may play an integrative role in the control of energy metabolism in hibernating jerboas (Jaculus orientalis).

\section{Heart}

Despite the low $\mathrm{Tb}$ and the reduction in heart rate during torpor, heart continues to function efficiently (Milsom et al., 1999). During hibernation, the heart relies on fatty acid catabolism instead of carbohydrate catabolism. This change is accompanied by differential gene expression in the heart. For example, mRNA transcripts encoding pyruvate dehydrogenase kinase (PDK) isozyme 4 are elevated and increased levels of the gene product inhibit the activity of pyruvate dehydrogenase, a key enzyme of carbohydrate oxidation (Andrews et al., 1998). Other hibernation-responsive genes that are up-regulated in heart of ground squirrels include the heart type fatty acid binding protein (H-FABP) (Hittel and Storey, 2001; Eddy and Storey, 2004), the mitochondriallyencoded subunit 2 of NADH-ubiquinone oxidoreductase (ND2), and the ventricular 
isoform of myosin light chain 1 (MLC1v); this latter response suggests that restructuring of myosin subunit composition could contribute to the changes in muscle contractility needed for hypothermic function (Fahlman et al., 2000). Up-regulation of all these genes highlights the importance of lipid-fueled metabolism to support the specialized needs of hibernation.

\section{Skeletal muscle}

It is well known that skeletal muscle disuse leads to atrophy, a pathological change resulting in a loss of muscle mass and a decrease in oxidative capacity in skeletal muscle. Examples of atrophy in humans include the loss of muscle mass during confined bed rest or space flight conditions. During hibernation, small animals such as ground squirrels, experience very little physical activity but yet they show very little muscle atrophy over the long period of muscle disuse (Wickler et al., 1991). Preliminary evidence identified a number of physiological changes that might help the skeletal muscle of hibernators to avoid disuse atrophy in order to maintain their key function in the process of shivering thermogenesis during arousal. Differential gene expression may represent an adaptive strategy used to preserve muscle mass through the hibernating period. Changes at the gene level include up-regulation of selected mitochondrialencoded genes, as well as up-regulation of genes similar to those identified in heart such as H-FABP (Hittel and Storey, 2001), MLC1v and ND2 (Fahlman et al., 2000), PDK4 (Buck et al., 2002).

The striking decrease of $\mathrm{Tb}$, the extreme reduction in metabolic rate, heart rate and oxygen consumption, creates conditions that would constitute severe ischemia in 
nonhibernating mammals, including man, but are noninjurious to hibernators. Therefore, hibernating ground squirrels represent an excellent research model to use for investigating a number of medically important problems including hypothermia resistance, cold preservation of transplantable organs, and the prevention of damage to the heart, brain and other ischemia-sensitive tissues during heart attack or stroke. By understanding the patterns of gene expression in mammalian hibernation, we can gain knowledge of both the key metabolic problems faced by different organs and the natural solutions that have been developed.

\section{OBJECTIVES AND HYPOTHESES}

\section{ER stress and the unfolded protein response}

During hibernation, organs of thirteen-lined ground squirrels experience a variety of conditions that in nonhibernating species would cause severe stress; these include hypothermia, oxidative stress, ischemia/reperfusion and hypoxia. Any of these in nonhibernating mammals could disrupt normal protein synthesis and processing by the endoplasmic reticulum resulting in the accumulation of misfolded or unfolded proteins. This is collectively called ER stress and the metabolic response to ER stress is known as the unfolded protein response (UPR). Although net protein synthesis is strongly reduced during hibernation, organs must still have a way to defend themselves against disruption of their protein synthesizing machinery, perhaps particularly against the effects of temperature change which is well-known to disrupt protein folding and conformation. A key response to ER stress in nonhibernating species is the up-regulation of the ER resident chaperone protein, glucose-regulated protein 78 (GRP8). 
Hypothesis 1: Organ responses to hibernation will include up-regulation of GRP78, a hallmark of ER stress, at both gene and protein levels in order to contribute to homeostasis of the protein synthesizing machinery during torpor.

Chapter 2 tests this hypothesis by examining the gene and protein expression of GRP78 using relative RT-PCR and Western blotting. Differentially expression of GRP78 was particularly evident in BAT and brain of torpid ground squirrels. Additionally, the use of electromobility shift and supershift assays provided a mechanism of grp78 gene regulation in brain, correlating grp 78 transcript levels with the increased binding of the activating transcription factor 4 (ATF4) in the grp78 promoter region. Furthermore, comparison of the full deduced amino acid sequence of ground squirrel GRP78 to the sequence of nonhibernating mammals revealed specific amino acid substitutions that may aid in sustaining the function of the protein at low $\mathrm{Tb}$ during torpor.

A large amount of research has established that perturbations of ER function due to various conditions including the accumulation of unfolded proteins in the ER lumen triggers the UPR which results in the coordinated up-regulation of a variety of genes, most prominently GRP78. Given that GRP78 was elevated in BAT and brain of torpid ground squirrels, I predicted that one or more of the transcription factors that are known to be involved in the UPR in nonhibernating species, would mediate the hibernation response.

Hypothesis 2: Transcription factors, including ATF4, that are involved in the UPR will be up-regulated during hibernation along with various downstream targets in order to mediate an integrated stress response that protects cells during torpor.

Chapter 3 examines the organ-specific expression of ATF4 as well as responses by 
alternate transcription factors and other downstream targets proteins known to be involved in the UPR including pPERK, ATF6 (50 kDa), CREB-1, p-CREB1, CHOP and GADD34. Western blotting and relative RT-PCR were used to show that whereas ATF4 protein levels increased significantly in BAT, brain and skeletal muscle, transcript levels remained constant during hibernation. Western blotting found no change of pPERK levels but revealed differential expression of ATF6 (50 kDa), CREB-1, CHOP and GADD34. Furthermore, levels of p-CREB1, the nuclear co-factor of ATF4, increased significantly in all four organs tested and moved with ATF4 in the nucleus to enhance transcriptional activity of downstream target genes during torpor. A partial sequence of atf4 was also obtained, including the 3 ' terminus. Comparison of the deduced amino acid sequence of squirrel ATF4 with sequences of nonhibernators revealed distinct amino acid substitutions that could aid ATF4 transcriptional function at the low Tb during torpor.

\section{Muscle atrophy}

Hibernating mammals experience long periods (weeks at a time) without locomotor activity that in nonhibernating species would result in severe atrophy of skeletal muscles. However, available evidence from several hibernating rodents indicates that skeletal muscle shows only slight atrophy and, furthermore, that the cardiac muscle maintains its mass (Wickler et al., 1991). Hence, I predicted that specific molecular mechanisms are invoked to minimize or prevent atrophy in hibernator muscle.

Hypothesis 3: Selected genes/proteins known to be involved in mediating muscle disuse atrophy will be down-regulated during torpor to minimize atrophy and help defend skeletal muscle functions. 
Chapter 4 investigates the hibernation-responsive expression of genes/proteins involved in muscle atrophy. The transcription factor, FoxOla, is involved in the regulation of skeletal muscle mass (Kamei et al., 2004) and in the development of muscle atrophy by inducing atrophy-related genes, including the atrophy-related ubiquitin ligase atrogin-1, also known as Muscle Atrophy F box (MAFbx) (Stitt et al., 2004; Sandri et al., 2004). Analysis of FoxOla found increased phosphorylation of this transcription factor in torpid animals and decreased or unchanged DNA binding activity, both supporting an inactivation of FoxO1a during torpor. Western blotting was also used to assess protein levels of the downstream gene, MAFbx/atrogin-1. The NFkB regulatory pathway is also involved with muscle atrophy and components of this pathway including NFkB p50 and Bcl-3 were measured in heart and muscle to assess their involvement in the regulation of muscle mass during hibernation.

\section{$\underline{\text { Apoptosis }}$}

Apoptosis or programmed cell death is a normal part of development and differentiation in multicellular animals and also mediates cell death in response to a wide variety of stresses. Several stress conditions that are known to trigger apoptosis in nonhibernating mammals such as temperature extremes, oxidative stress and ischemia/reperfusion are naturally occurring features of the torpor-arousal cycles of hibernation (Kurtz et al., 2006). Hence, I predicted that the suppression of stressmediated apoptosis would be a valuable mechanism to help ground squirrel cells and organs to make transitions to/from the torpid state.

Hypothesis 4: Apoptosis is inhibited/suppressed during torpor via altered 
expression of anti-apoptotic and pro-apoptotic proteins in hibernator organs.

Chapter 5 uses Western blotting to analyze the relative levels of two main proteins that have anti-apoptotic functions ( $\mathrm{Bcl}-\mathrm{XL}$ and $\mathrm{Bcl}-2)$ as well as the proapoptotic protein, $\mathrm{BAD}$, in ground squirrel organs. $\mathrm{BAD}$ is controlled by reversible phosphorylation and so the relative amounts of Ser 112, Ser 136 and Ser155 phosphorylated BAD were also quantified. Transcript levels of $b c l-x l$ were also measured via RT-PCR to determine whether gene regulation was responsible for hibernationresponsive changes in protein content. 
Fig. 1.1: Body temperature (Tb) of a golden-mantled ground squirrel (S. lateralis) as a function of time over a one year period, including the winter hibernation season. The enlargement shows a region encompassing three torpor-arousal cycles. The different stages are entrance into torpor (EN) lasting $\sim 12 \mathrm{~h}$; early torpor (ET) lasting $\sim 48 \mathrm{~h}$; late torpor (LT) about 7-14 days; arousal (AR) lasting $\sim 2 \mathrm{~h}$, and interbout (IBA) with duration of $\sim 20 \mathrm{~h}$. This figure is taken from Carey et al. (2003a). 


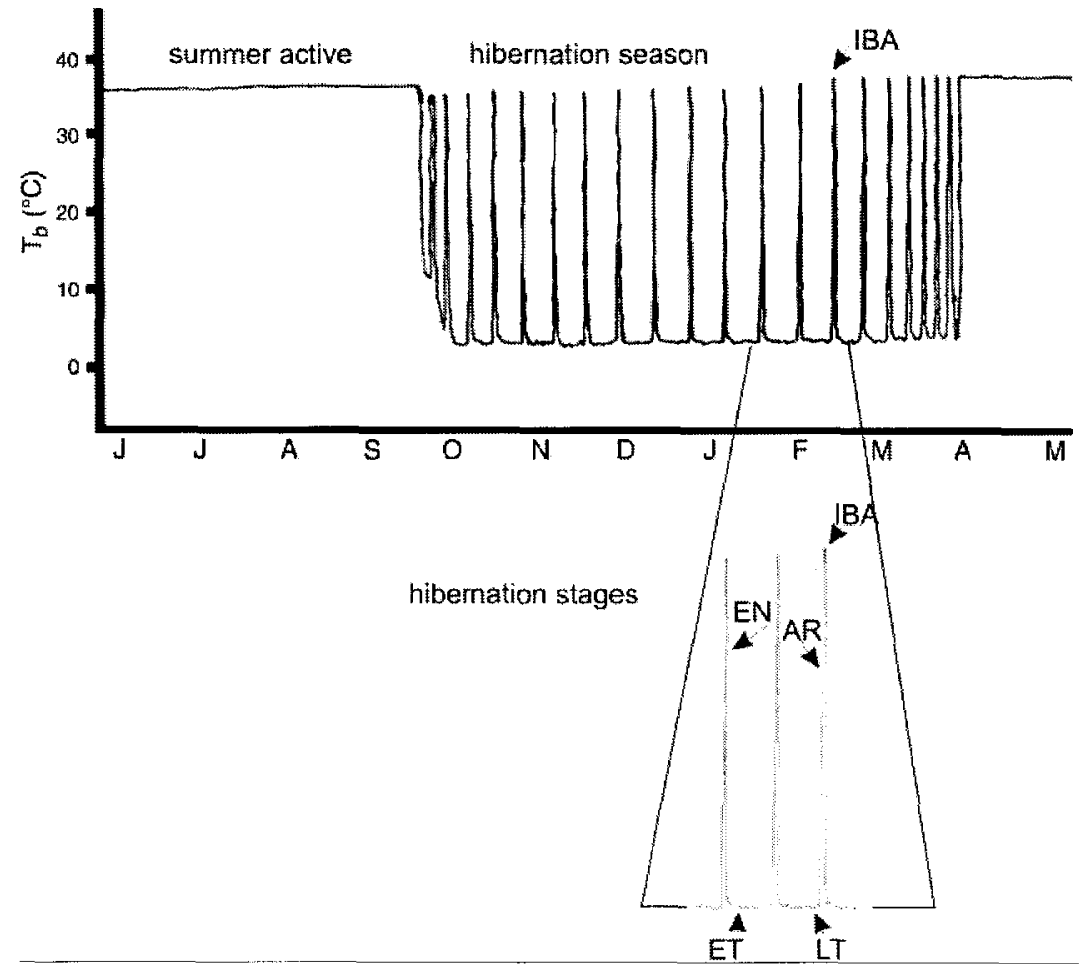

Reproduced with permission of the copyright owner. Further reproduction prohibited without permission. 
Fig. 1.2: Distribution map for thirteen-lined ground squirrels, S. tridecemlineatus. The range of thirteen-lined ground squirrels extends from Central Alberta, Manitoba, and Saskatchewan in the North to Texas and New Mexico in the South, and from Central Ohio in the east to Colorado in the west. Taken from Cleary and Craven (1994). 


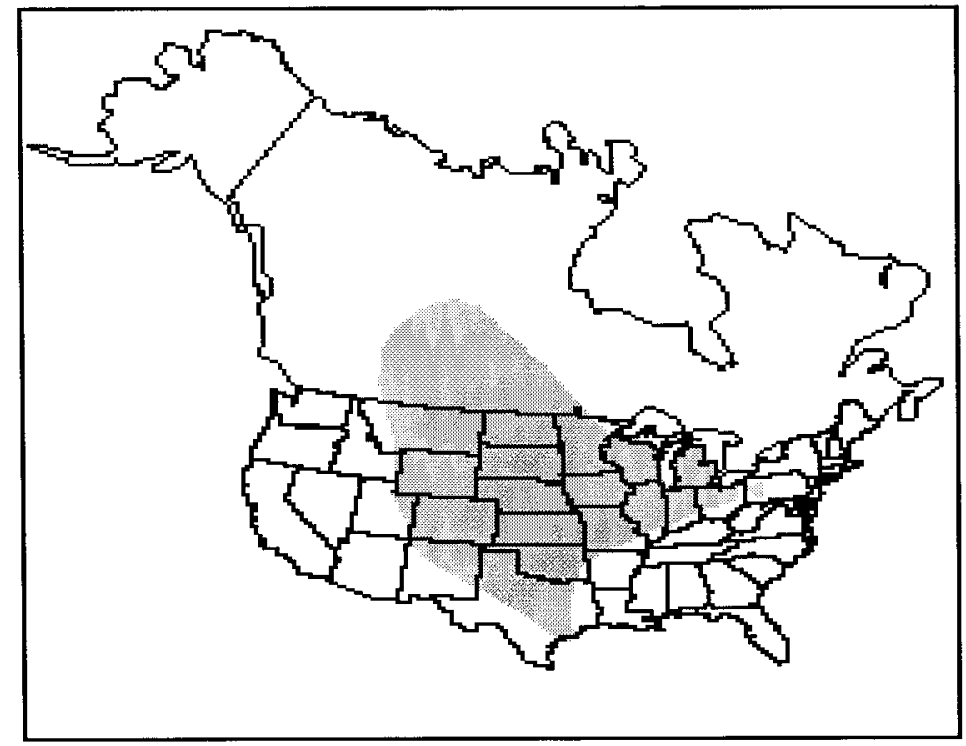




\section{Chapter 2}

\section{Up-regulation of the endoplasmic reticulum}

\section{molecular chaperone GRP78 during hibernation in thirteen-lined ground squirrels}




\section{INTRODUCTION}

As mentioned in Chapter 1, mammalian hibernation is an energy-conservation strategy that includes biochemical, physiological and behavioral adjustments that allow animals to live for extended periods of time at very low metabolic rates (often $1-5 \%$ of normal) and low core body temperatures (often near $0^{\circ} \mathrm{C}$ ) (Wang and Lee, 1996). Mechanisms that support hibernation include a profound suppression of overall metabolic rate, a reorganization of cell priorities for ATP use to preferentially turn down many nonessential ATP-consuming cell functions, and enhanced expression of selected genes whose products aid long term survival in the torpid state (Storey, 2003; Storey, 2004). One of the ATP-consuming functions that is suppressed in hibernator organs is protein synthesis; this is accomplished by mechanisms including polysome disaggregation and reversible phosphorylation control of ribosomal initiation and elongation factors (Storey, 2003; Storey and Storey, 2004). However, selective synthesis of some proteins continues; for example, increased expression of multiple proteins involved in lipid transport/catabolism and mitochondrial energy metabolism has been documented at both gene and protein levels during hibernation (Storey, 2003 and 2004; Storey and Storey, 2004; Eddy and Storey, 2004 and Eddy et al., 2005a).

Protein synthesis during hibernation occurs under cellular conditions that are very different from the normal situation for mammals. For example, body temperature (Tb) can vary over at least $35^{\circ} \mathrm{C}$. Temperature change not only alters reaction rates but also affects the strength of the weak bonds that are critical to the conformation of macromolecules, the conformational changes that occur during ligand binding and catalysis in enzymes, and various macromolecular interactions such as proteinprotein, protein-DNA, and protein-membrane binding. Such effects of low temperature could alter critical steps in the synthesis and processing of new proteins. 
Arousal from torpor also generates significant oxidative stress associated with the tremendously rapid increase in oxygen uptake and oxygen consumption that is needed to fuel thermogenesis (Barja de Quiroga, 1992; Carey et al., 2000). These stresses in nonhibernating mammals can cause protein misfolding and saturation of the folding pathways in the endoplasmic reticulum (ER) creating a condition called the unfolded protein response (UPR), an intracellular signaling pathway that is induced to return the ER to its normal physiological state (Schröder and Kaufman, 2005). Hence, I predicted that hibernators would need to have mechanisms to minimize protein misfolding during protein synthesis under the unusual cellular conditions of the hibernating state. One of these mechanisms could be the enhanced production of molecular chaperones such as the $78 \mathrm{kDa}$ glucose regulated protein (GRP78).

GRP78 is a constitutively expressed protein that is a member of the heat shock protein 70 family of molecular chaperones. It resides in the ER and is involved in the folding and assembly of newly synthesized proteins (Kozutsumi et al., 1988; Gething, 1999; Lee, 2001). GRP78 also has an anti-apoptotic function to prevent ER stressinduced cell death (Gething, 1999) and it is used as a biomarker for the onset of the UPR (Lee, 2001). Various physiological conditions and stimuli such as glucose starvation, calcium depletion, inhibition of $\mathrm{N}$-glycosylation, reduction of disulfide bonds and accumulation of malfolded protein in the ER lumen, are capable of disrupting endoplasmic reticulum (ER) function and triggering ER stress. ER stress is therefore followed by the induction of the UPR (Gething 1999; Lee, 2001). A model of the role of GRP78 in modifying UPR signaling and cell survival is summarized in Fig. 2.1. The present study examines mRNA and protein expression of GRP78 in seven organs of hibernating thirteen-lined ground squirrels, Spermophilus tridecemlineatus, documenting strong hibernation-responsive up-regulation of the 
protein in two key oxygen-sensitive organs - brain and brown adipose tissue (BAT). I further examined the transcriptional control of ground squirrel grp78, providing evidence that transcriptional activation of the $g r p 78$ promoter during hibernation may be linked to the activating transcription factor 4 (ATF4).

\section{MATERIALS AND METHODS}

\section{Animal experiments and tissue collection}

Thirteen lined ground squirrels, Spermophilus tridecemlineatus (130-180 g), were captured by a licensed trapper (TLS Research, Michigan) and transported to the Animal Hibernation Facility at the National Institute of Neurological Disorders and Stroke (NIH, Bethesda, MD). All animal experiments were conducted by the laboratory of Dr. J.M. Hallenbeck using their standard protocols (Chen et al., 2001) that are approved by the NIH Institutional Animal Care and Use Committee. Briefly, animals were kept on a fall day/night light cycle in shoebox cages maintained at $21^{\circ} \mathrm{C}$ and fed ad libitum until they entered and finished the pre-hibernation phase of hyperphagia that maximizes lipid stores. A sensor chip was introduced under the squirrel skin and the $\mathrm{Tb}$ of each animal was monitored electronically. When squirrels had reached a plateau weight of 220-240 g, they were placed in their cages containing wood shavings into a cold chamber at $4^{\circ} \mathrm{C}$ and $60 \%$ humidity. The hibernaculum was kept in constant darkness, except for a photographic red safe light (3-5 lux), and could be entered only through a darkened anteroom. Noise within the chamber was kept to a minimum. Individuals settled into hibernation after different lengths of time but all were sampled on the same day after each individual had been hibernating for $2-5$ days (as indicated by continuous $\mathrm{Tb}$ readings of $\sim 6^{\circ} \mathrm{C}$ ). Animals that had not entered torpor after at least 3 days in the cold room and that showed continuous high $\mathrm{Tb}\left(36-38^{\circ} \mathrm{C}\right)$ 
were sampled as controls. All animals were killed by decapitation and tissues were excised, frozen immediately in liquid nitrogen and transported to Ottawa on dry ice where they were placed at $-80^{\circ} \mathrm{C}$ until use.

\section{Total RNA extraction}

All materials and solutions were treated with $0.1 \% \mathrm{v} / \mathrm{v}$ diethylpyrocarbonate (DEPC) and autoclaved prior to use. Total RNA was isolated from tissues of control and hibernating ground squirrels using Trizol $^{\mathrm{TM}}$ reagent (Invitrogen), essentially following manufacturer's instructions. Briefly, $100 \mathrm{mg}$ of tissue was homogenized in $1 \mathrm{~mL}$ Trizol using a Polytron homogenizer followed by the addition of $200 \mu \mathrm{L}$ of chloroform and then centrifugation at $10,000 \times \mathrm{g}$ for $15 \mathrm{~min}$ at $4^{\circ} \mathrm{C}$. The upper aqueous phase that contains total RNA was removed to a fresh tube and RNA was precipitated by adding $500 \mu \mathrm{L}$ of isopropanol followed by incubation at room temperature for $10 \mathrm{~min}$. After a second centrifugation at $10,000 \mathrm{x}$ f for $15 \mathrm{~min}$ at $4^{\circ} \mathrm{C}$, the total RNA pellet was washed with $1 \mathrm{~mL}$ of $70 \%$ ethanol and then centrifuged as above. The supernatant was removed and the pellet was air-dried for 10-15 min. A volume of $70 \mu \mathrm{L}$ DEPC-treated water was added to resuspend the RNA pellet. Three samples of each tissue were separately extracted. The quality of total RNA was judged based on the ratio of absorbances at $260 \mathrm{~nm}$ and $280 \mathrm{~nm}$. In addition, all RNA samples were assessed using $1 \%$ formaldehyde agarose gel electrophoresis with ethidium bromide staining to check for the integrity of $18 \mathrm{~S}$ and $28 \mathrm{~S}$ ribosomal RNA (rRNA) bands. 


\section{First strand cDNA synthesis and PCR amplification}

Approximately $10-15 \mu \mathrm{g}$ of total RNA was diluted with DEPC water to $10 \mu \mathrm{L}$ final volume and used to synthesize cDNA. Briefly, $1 \mu \mathrm{L}$ of $200 \mathrm{ng} / \mu \mathrm{L}$ oligo-dT (5'TTTTTTTTTTTTTTTTTTTTTTV-3'; V=A or G or C) (Sigma Genosys) that forms a hybrid with the poly A tail of mRNA was added to the RNA sample and placed in a $68^{\circ} \mathrm{C}$ water bath for $5 \mathrm{~min}$. The mixture was then chilled rapidly on ice and $4 \mu \mathrm{L} 5 \mathrm{X}$ first strand buffer, $2 \mu \mathrm{L} 10 \mathrm{mM}$ DTT, $1 \mu \mathrm{L}$ dNTPs and $1 \mu \mathrm{L}$ reverse transcriptase enzyme Superscript II (all reagents from Invitrogen) were added for a total volume of $19 \mu \mathrm{L}$. The mix was incubated at $42^{\circ} \mathrm{C}$ for $45 \mathrm{~min}$ then held at $4^{\circ} \mathrm{C}$. The resulting cDNAs from both control and hibernating samples were serially diluted $\left(10^{-1}, 10^{-2}\right.$, $10^{-3}, 10^{-4}$, and $10^{-5}$ ) and amplified by polymerase chain reaction (PCR) with grp78 primers that were designed from the consensus sequence of mammalian grp 78 , using the Primer Designer program, version 3.0 (Scientific and Educational Software). The nucleotide sequences of the grp78 primer pairs were 5' AAGAACGGCCGCGTGGAGAT - 3' and 5' -CTCTTATCCAGGCCATAATGC 3'. The PCR reaction of $25 \mu \mathrm{L}$ final volume was composed by mixing $14.37 \mu \mathrm{L}$ of sterile water, $5 \mu \mathrm{L}$ of diluted cDNA, $1.25 \mu \mathrm{L}$ of primer mixture $(0.5 \mu \mathrm{M}$ forward and $0.5 \mu \mathrm{M}$ reverse), $2.5 \mu \mathrm{L}$ of $10 \mathrm{X}$ PCR buffer (Invitrogen), $1.25 \mu \mathrm{L}$ of $50 \mathrm{mM} \mathrm{MgCl}$, $0.5 \mu \mathrm{L}$ of $10 \mathrm{mM}$ dNTPs and $0.13 \mu \mathrm{L}$ of Taq Polymerase (Invitrogen). The PCR started with an initial step of $3 \mathrm{~min}$ at $94^{\circ} \mathrm{C}$ followed by 35 cycles of $30 \mathrm{sec}$ at $94^{\circ} \mathrm{C}$, $30 \mathrm{sec}$ at $57^{\circ} \mathrm{C}$, and $30 \mathrm{sec}$ at $72^{\circ} \mathrm{C}$. The final step was $72^{\circ} \mathrm{C}$ for $7 \mathrm{~min}$. The housekeeping gene $\alpha$-tubulin was amplified as an internal control; the primers used for this were forward (5' - AAGGAAGATGCTGCCAATAA - 3') and reverse (5' GGTCACATTTCACCATCTG - 3'). PCR products were separated on a $1 \%$ agarose gel, stained with ethidium bromide, and the intensity of the most dilute cDNA sample 
that was visible was used for quantification purposes to make sure that the products had not reached their amplification saturation. Hence, RT-PCR results were taken from the linear amplification range of the progress logarithmic curve (the exponential phase PCR signal strength for both grp78 and $\alpha$-tubulin gene at 35 cycles). Quantification of the band intensity on the gel was performed using a ChemiGenius Bio Imaging System (SynGene) and Syngene tools software (Syngene, MD, USA). RT-PCR values are presented as a ratio of the grp78's signal in the selected linear amplification cycle divided by the $\alpha$-tubulin signal in the specific condition. The relative mRNA levels for hibernating versus euthermic states were calculated as followed:

$$
\text { Relative mRNA content }=\frac{\text { (hibernating grp } 78 / \text { hibernating } \alpha \text {-tubulin })}{\text { (euthermic grp } 78 / \text { euthermic } \alpha \text {-tubulin })}
$$

The mean ratios of hibernating to euthermic values (relative mRNA content) and the S.E.M on the ratios were then calculated, and the data are shown as mean ratios \pm S.E.M in histograms.

\section{Rapid amplification of cDNA ends (5' and 3' RACE)}

A partial cDNA sequence of ground squirrel $\operatorname{grp} 78$ (465 bp) was obtained by RT-PCR. To retrieve the full cDNA sequence, the technique of RACE was used. The 5' end of $g r p 78$ was amplified using two anti-sense gene specific primers (GSP) designed from the partial sequence. The nucleotide sequences of the primers were: 5 , - GGTCACATTTCACCATCTG - 3' for GSP1 and 5'GAGGTGAGCTGCTTCTTGACTGTATC- 3' for GSP2. The protocol used was as described by Gibco BRL with a few modifications. Briefly, $1 \mu \mathrm{g}$ of total RNA from BAT of hibernating ground squirrels was reverse transcribed to cDNA in a sterile 
microcentrifuge tube containing the following in a final volume of $25 \mu \mathrm{L}: 2.5 \mu \mathrm{L}$ of GSP1 $(1 \mu \mathrm{M}), 2.5 \mu \mathrm{L}$ of $10 \mathrm{X}$ PCR buffer, $2.5 \mu \mathrm{L}$ of $25 \mathrm{mM} \mathrm{MgCl}_{2}, 1 \mu \mathrm{L}$ of $10 \mathrm{mM}$ dNTPs and $1 \mu \mathrm{L}$ of reverse transcriptase MMLV-RT (Invitrogen). After an incubation of $50 \mathrm{~min}$ at $42^{\circ} \mathrm{C}$, the tube was placed at $70^{\circ} \mathrm{C}$ for $15 \mathrm{~min}$ to denature the reverse transcriptase. The mixture was collected from the side of the tube by brief spinning and then $1 \mu \mathrm{L}$ of $2 \mathrm{U} / \mu \mathrm{L}$ RNase $\mathrm{H}$ was added followed by incubation for $30 \mathrm{~min}$ at $37^{\circ} \mathrm{C}$ and then placing the sample on ice. To purify the cDNA, $2.5 \mu \mathrm{L}$ of $3 \mathrm{M}$ sodium acetate $\mathrm{pH} 5.3$ and $25 \mu \mathrm{L}$ isopropanol (Sigma) were added and the mixture was incubated for $2 \mathrm{~h}$ at $-20^{\circ} \mathrm{C}$ followed by centrifugation at $10,000 \mathrm{x} \mathrm{g}$ for $20 \mathrm{~min}$, and retrieval of the pellet which was resuspended in sterile water. A dC tail was then added onto the cDNA by incubation with $2 \mathrm{mM}$ dCTP and a terminal deoxynucleotidyl-terminal transferase according to the manufacturer's protocol (Invitrogen). Next, PCR was performed using the dC-tailed cDNA with GSP2 and a universal primer AAP (5'- GGCCACGCGTCGACTAGTACGGGGGGGGGG- 3') (Sigma Genosys). Cycles for amplification were $3 \mathrm{~min}$ at $94^{\circ} \mathrm{C}$ for the initial step followed by 35 cycles of $94^{\circ} \mathrm{C}$ for $1 \mathrm{~min}, 55^{\circ} \mathrm{C}$ for $1 \mathrm{~min}, 72^{\circ} \mathrm{C}$ for 1 min with a final step of $72^{\circ} \mathrm{C}$ for $7 \mathrm{~min}$. The resulting PCR product was diluted 100 times with sterile water and $2.5 \mu \mathrm{L}$ was used in a second round of PCR as described above with grp78 gene specific primer and the universal primer AUAP (5, GGCCACGCGTCGACTAGTAC- 3') (Sigma Genosys ). The PCR products were separated on a $1 \%$ agarose gel to confirm the amplification of a cDNA with the expected size.

The 3' end was amplified using a Takara 3' RACE kit (Promega). Total RNA ( $1 \mu \mathrm{g}$ ) was reverse transcribed into cDNA according to the manufacturer's instructions, using an oligo-dT-3 sites primer to initiate the first strand synthesis. The 
resulting cDNA was amplified with grp78 gene specific primer (5' CAGCTGCTATTGCTTATGGCCTGGAT- 3') and the 3-sites adaptor primer (Promega). After performing 35 cycles of PCR $\left(94^{\circ} \mathrm{C}\right.$ for $1 \mathrm{~min}, 58^{\circ} \mathrm{C}$ for $1 \mathrm{~min}, 72^{\circ} \mathrm{C}$ for $1 \mathrm{~min}$ ) and a final extension at $72^{\circ} \mathrm{C}$ for $7 \mathrm{~min}$, the PCR products were separated on a $1 \%$ agarose gel and analyzed as above.

\section{Sequencing}

RT-PCR and RACE products were sequenced by either Canadian Molecular Research Services (Ottawa, ON) or by CORTEC (Kingston, ON). Sequences were verified as encoding the grp78 using the program BLASTN (http://www.ncbi.nlm.nih.gov/blast) at the NIH. The deduced amino acid sequences were also verified as being GRP78 by using the program BLASTP at the same site. The sequence was submitted to GenBank with the accession number DQ166628.

\section{Protein extraction and Western blot analysis}

Western blotting was used to examine the levels of GRP78 protein in tissue samples from euthermic and hibernating animals. Cell-free extracts were prepared by homogenizing frozen tissue samples $(500 \mathrm{mg})$ using a Polytron homogenizer and 1 $\mathrm{mL}$ of homogenizing buffer containing $20 \mathrm{mM}$ Tris-base, $150 \mathrm{mM} \mathrm{NaCl}, 1 \mathrm{mM}$ EDTA, $1 \mathrm{mM}$ EGTA, $1 \mathrm{mM} \mathrm{NaF,} 10 \mathrm{mM}$ ß-glycerophosphate, 1\% v/v Triton X-100 and $1 \mathrm{mM}$ phenylmethylsulfonyl fluoride. After a $15 \mathrm{~min}$ centrifugation at $10,000 \times \mathrm{g}$, supernatants were removed and soluble protein levels were determined using the BioRad protein assay (BioRad, Hercules, CA) with bovine serum albumin (BSA) as the standard. Aliquots of supernatant were then mixed 1:1 v/v with 2X SDS-PAGE sample buffer (100 mM Tris-HCl (pH 6.8), 4\% w/v SDS, $20 \%$ v/v glycerol, $0.4 \% \mathrm{w} / \mathrm{v}$ 
bromophenol blue) and $10 \% \mathrm{v} / \mathrm{v} 2$ 2-mercaptoethanol, boiled and stored at $-20^{\circ} \mathrm{C}$ until use. Equal amounts of protein from control and hibernating lysates (10-30 $\mu \mathrm{g}$ depending on the experiment) were then layered on 10 or $12 \%$ SDS polyacrylamide gels $(10 \%$ or $12 \%$ acrylamide, $0.4 \mathrm{M}$ Tris (pH 8.8 ), $0.1 \%$ SDS, $0.1 \%$ APS, $0.04 \%$ TEMED), covered with a 5\% stacking gel (5\% acrylamide, $0.13 \mathrm{M}$ Tris (pH 6.8), $0.1 \%$ SDS, $0.1 \%$ APS, $0.1 \%$ TEMED) and separated at $180 \mathrm{~V}$ for $1 \mathrm{~h}$. Kaleidoscope prestained molecular mass markers (Bio-Rad) were used to estimate the size and positions of proteins on the gel. Proteins were electroblotted onto polyvinylidene difluoride (PVDF) membranes (Pall) by wet transfer with pre-chilled solution containing $25 \mathrm{mM}$ Tris ( $\mathrm{pH} 8.5$ ), $192 \mathrm{mM}$ glycine, and $20 \% \mathrm{v} / \mathrm{v}$ methanol at $4^{\circ} \mathrm{C}$ for 2 $\mathrm{h}$ at $70 \mathrm{~V}$. After the transfer of proteins, membranes were blocked for $1 \mathrm{~h}$ in Tris buffered saline containing Tween-20 (TBST: $20 \mathrm{mM}$ Tris base, $140 \mathrm{mM} \mathrm{NaCl}, 0.05 \%$ v/v Tween-20) with $2.5 \%$ non-fat dried milk added. The blots were then incubated at $4^{\circ} \mathrm{C}$ overnight with primary antibody diluted 1:20000 v/v in TBST; the primary was rabbit anti-GRP78 (BiP) polyclonal antibodies (Stressgen). After incubation, blots were washed several times with TBST and then incubated at room temperature for $2 \mathrm{~h}$ with a 1:2000 v/v dilution of secondary antibody, anti-rabbit IgG conjugated to horseradish peroxidase (Cell Signaling). Detection of signal on the PVDF membrane was done using an enhanced chemiluminescence (ECL) system as recommended by the manufacturer (Pierce). The membrane was scanned using the SynGene and the resulting image was analyzed with SynGene tools software (Syngene, MD, USA). Equal protein loading was confirmed by Coomassie blue staining of the membrane.

\section{Two-Dimensional Polyacrylamide Gel electrophoresis (2D-gel)}

Two-dimensional gel electrophoresis was performed to demonstrate that the 
rabbit anti-GRP78 polyclonal antibodies used in Western blotting were specific to GRP78 protein or GRP78 peptide target. This technique has two components: isoelectric focusing (IEF) and SDS-PAGE. The first dimension (IEF) was carried out in an acrylamide matrix cast in small capillary tubes where the proteins are separated based on their isoelectric point (pI). The IEF gel contained $9.2 \mathrm{M}$ urea, 4\% acrylamide, $1.6 \%$ (v/v) ampholytes $\mathrm{pH} 5-8$ and $0.4 \%$ ampholytes $\mathrm{pH} 3.5-10$ (Sigma), $0.01 \%$ ammonium persulfate, and $0.1 \%$ TEMED. The upper chamber running buffer

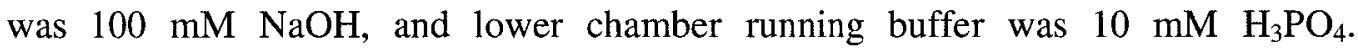
Following polymerization, the capillary gels were pre-electrophoresed for $10 \mathrm{~min}$ at $200 \mathrm{~V}, 15 \mathrm{~min}$ at $300 \mathrm{~V}$ and $15 \mathrm{~min}$ at $400 \mathrm{~V}$. First dimension sample buffer contained 9.5 $\mathrm{M}$ urea, 5\% $\beta$-mercaptoethanol, $1.6 \%$ ampholytes ( $\mathrm{pH} 5-8$ range), and 0.4\% ampholytes ( $\mathrm{pH} 3.5-10$ range). Total soluble protein, extracted from skeletal muscle of euthermic squirrels as described above, was mixed with the first dimension sample buffer $(1: 1 \mathrm{v} / \mathrm{v})$ and allowed to incubate at room temperature for $15 \mathrm{~min}$. An aliquot of $10 \mu \mathrm{g}$ proteins was loaded into the capillary sample reservoir and overlaid with first dimension sample overlay buffer $(9 \mathrm{M}$ urea, $0.8 \% \mathrm{pH} 5-8$ ampholytes, $0.2 \% \mathrm{pH} 3.5$ 10 ampholytes, $0.005 \% \mathrm{w} / \mathrm{v}$ bromophenol blue). Separation occurred over $10 \mathrm{~min}$ at $500 \mathrm{~V}$ and then $3 \mathrm{~h}$ at $750 \mathrm{~V}$. Following isoelectric focusing the gel was removed from the capillary tube and incubated for $10 \mathrm{~min}$ in SDS equilibration buffer containing $62.5 \mathrm{mM}$ Tris- $\mathrm{HCl}, \mathrm{pH} 6.8,2.3 \% \mathrm{w} / \mathrm{v}$ SDS, $5.0 \% \quad \beta$-mercaptoethanol, $10 \% \mathrm{w} / \mathrm{v}$ glycerol and $0.00125 \% \mathrm{w} / \mathrm{v}$ bromophenol blue. The second dimension (SDS-PAGE) involves separating the proteins from the IEF gel tube according to molecular weight. The first dimension gel was sealed with a sealing gel $(1 \% \mathrm{w} / \mathrm{v}$ agarose in SDS equilibration buffer), at the top of a $10 \%$ acrylamide separating gel. Separation and transfer onto PVDF membrane, and the rest of the procedure was the same as 
described above in Western blotting analysis, using the rabbit anti-GRP78 polyclonal antibodies.

\section{Nuclear extract preparation and electrophoretic mobility shift assay (EMSA)}

Nuclear extracts from brain of euthermic and hibernating ground squirrels were prepared using a slight modification of the method described by Dignam et al. (1983). Briefly, $0.5 \mathrm{~g}$ of tissue was disrupted at $4^{\circ} \mathrm{C}$ in $1 \mathrm{~mL}$ homogenization buffer (10 mM HEPES, pH 7.9, $10 \mathrm{mM} \mathrm{KCl,} 10 \mathrm{mM}$ EDTA, $10 \mathrm{mM}$ DTT, and $1.5 \mu \mathrm{L}$ protease inhibitor cocktail (Sigma) using a Dounce homogenizer. Nuclei were pelleted by centrifugation at $8000 \mathrm{x}$ g for $10 \mathrm{~min}$. The supernatant, which represents the cytoplasmic extract, was removed and the pellet was washed with the same buffer before being resuspended in $150 \mu \mathrm{L}$ of extraction buffer (20 mM HEPES, $\mathrm{pH} 7.9,400$ $\mathrm{mM} \mathrm{NaCl}, 1 \mathrm{mM}$ EDTA, 10\% v/v glycerol, $10 \mathrm{mM} \mathrm{DTT}$, and $1.5 \mu \mathrm{L}$ protease inhibitor cocktail) and mixed at $4^{\circ} \mathrm{C}$ for $1 \mathrm{~h}$ with gently shaking. After centrifugation at $10,000 \mathrm{x} \mathrm{g}$ for $10 \mathrm{~min}$ the supernatant (nuclear extract) was collected. Protein concentration in the nuclear fraction was determined by the Bio-Rad protein assay. Since transcription factors are known to move inside the nucleus where they activate downstream genes, the separation of nuclear and cytoplasmic fractions is critical. To confirm the separation of these two fractions, $10 \mu \mathrm{g}$ of protein from cytoplasmic and nuclear extracts of both euthermic and hibernating animals were separated by SDSPAGE and then gels were subjected to Western blotting with anti-histone $\mathrm{H} 3$ antibody (Cell Signaling) at a dilution of $1 / 1000$, followed by anti-rabbit secondary antibody as above. The histone $\mathrm{H} 3$ band was found only in the nuclear extracts and not in the cytoplasmic extracts, thereby confirming the integrity of the nuclei when separated from the cytoplasmic fraction (data not shown). 
The electrophoretic mobility shift assay was performed as described by Roy and Lee (1999) with slight modifications and using radiolabeled synthetic oligonucleotides (probe) containing a sequence corresponding to a cis-acting element, the ATF4 binding site (5'- TGACGTGA-3') or ATF/CRE-like site in the grp78 promoter (Luo et al., 2003). The oligonucleotides used for probing were designed from the rat grp78 promoter with sequences as follows (ATF4 binding site is underlined): 5'- CGCGTACCAGTGACGTGAGTTGCGGAGG - 3' and the complementary strand $5^{\prime}$ - CCTCCGCAACTCACGTCACTGGTACGCG - 3'. Mutant probes were: 5'-CGCGTACCAGGTCGACTCGTTGCGGAGG - 3' and 5'CCTCCGCAACGAGTCGACCTGGTACGCG - 3' (mutated ATF4 binding site underlined). The above oligonucleotides were annealed into double-stranded form and were ${ }^{32} \mathrm{P} 5$ '-end-labeled with T4 polynucleotide kinase (New England Biolabs), using $5 \mu \mathrm{L} /$ reaction of $10 \mathrm{mCi} / \mathrm{mL}^{32} \mathrm{P}-\gamma-\mathrm{dATP}$ (Amersham). Prior to starting the binding reaction, the nuclear extract was digested with restriction enzymes that do not cut inside the promoter region used, EcoRI (Gibco BRL) and SmaI (New England Biolabs), to shear the genomic DNA contained in the nuclear extract. Equal amounts ( $20 \mu \mathrm{g}$ ) of digested nuclear extract from brain of euthermic and hibernating squirrels were incubated for $5 \mathrm{~min}$ at room temperature in $20 \mu \mathrm{L}$ of binding reaction with final concentrations of reagents: $50 \mathrm{mM} \mathrm{NaCl}, 10 \mathrm{mM}$ Tris- $\mathrm{HCl}(\mathrm{pH} 7.6), 1 \mathrm{mM}$ EDTA, $0.5 \mathrm{mM}$ dithiothreitol, $5 \% \mathrm{v} / \mathrm{v}$ glycerol and $0.3 \mu \mathrm{g}$ poly(dIdC). For specific and nonspecific competition with the labeled probe, $10 \mathrm{X}$ more unlabeled probe or mutant probe, respectively, were added to the binding reaction mixture before incubation. Then $160 \mathrm{ng}$ of double stranded, radiolabeled oligonucleotide probe was added to the reaction mixture and incubated for a further $20 \mathrm{~min}$ at room temperature. Antibodies against CREB-1, a cofactor of ATF4) as well as Bcl-2 (used as negative control) were 
purchased from Santa Cruz Biotechnology. The antibodies were then added to each reaction and incubated at room temperature for another $20 \mathrm{~min}$. DNA-protein complexes were separated from unbound DNA probe by electrophoresis on native 5\% polyacrylamide gel at $300 \mathrm{~V}(\sim 25 \mathrm{~mA})$ in $45 \mathrm{mM}$ Tris/borate $(\mathrm{pH} 8.0)$ containing 1 mM EDTA. The resolved gel was dried and exposed to a phosphor screen (BioRad).

\section{Statistical analysis}

Relative RT-PCR $(n=3)$ and Western Blotting $(n=3-5)$ data were gathered from independent tissue extracts from different animals, a total pool size of $n=6$ individuals being available for each treatment group. RT-PCR bands for $g r p 78$ were normalized relative to $\alpha$-tubulin bands obtained from the same cDNA reaction whereas Western blot bands specific to GRP78 protein were normalized against three Coomassie stained protein bands that did not change in intensity between euthermic and hibernating conditions. Data are expressed as means with corresponding SEM. Statistical testing of normalized band intensities from euthermic versus hibernating samples used the Student's $t$-test with significance levels of $\mathrm{P}<0.05$. The ratio hibernating/euthermic was then calculated and plotted in histograms. Error bars shown on histograms are the S.E.M. on the ratios of hibernating to euthermic values.

\section{RESULTS}

\section{Analysis of $g r p 78$ transcript levels}

Using forward and reverse primers designed from a consensus sequence of human, mouse and rat grp78 (GenBank accession numbers: NM_005347, NM_022310 and NM_013083 respectively), a 465 bp cDNA was amplified from

BAT of ground squirrels. After nucleotide sequencing, the cDNA was shown to 
encode $g r p 78$; the nucleotide sequence shared $94 \%$ identity with human $\operatorname{grp} 78 \mathrm{cDNA}$ and its deduced amino acid sequence was $99 \%$ identical with human GRP78 protein.

The primers were then used in a semi-quantitative RT-PCR to assess $g r p 78$ mRNA levels, relative to $\alpha$-tubulin levels, in seven tissues (BAT, brain, heart, kidney, liver, lung, skeletal muscle) of euthermic versus hibernating ground squirrels. Fig. 2.2A shows grp78 mRNA expression in each organ together with expression levels of $\alpha$-tubulin. Preliminary studies in our laboratory showed that $\alpha$-tubulin mRNA levels were unchanged in 13-lined ground squirrel tissues between euthermic and hibernating states. Transcript levels of $\operatorname{grp} 78$ were normalized against $\alpha$-tubulin amplified from the same samples, expression ratios were calculated, and mean ratios in hibernation versus euthermia are shown in Fig. 2.2B. Transcript levels of grp78 increased significantly $(\mathrm{P}<0.05)$ by 3.5 -fold in BAT and 4.1 -fold in brain of hibernating squirrels compared with euthermic controls but remained unchanged in all other tissues.

\section{Cloning of S. tridecemlineatus grp78 cDNA}

To retrieve the full cDNA sequence of ground squirrel grp78, GSPs were designed from the 465 bp partial grp 78 cDNA sequence and used in 5' and 3' RACE to extend and amplify the remaining portions of the ground squirrel grp 78 sequence. A final assembled cDNA sequence of 2490 bp contained the full open reading frame (ORF) of ground squirrel grp78 cDNA (Fig. 2.3) and was submitted to GenBank with accession number DQ166628. Translation of the ORF revealed a protein of 654 amino acids as in human and rat GRP78 (mouse GRP78 has 655 amino acids). Fig. 2.4 shows the alignment of ground squirrel GRP78 with the amino acid sequences of human, mouse and rat GRP78. The homology tree and homology matrix show that the ground squirrel GRP78 amino acid sequence shared 99\% identity to human and 98\% 
to mouse and rat GRP78 (Fig. 2.5A and B). Four amino acid substitutions were evident in the squirrel sequence as compared with the other mammalian species (residues shown in bold underline in Fig. 2.4). These substitutions were a valine at position 8 compared with methionine or alanine in the other species, a hydrophilic serine substitution at position 451 replacing a hydrophobic phenylalanine in the other sequences, and two substitutions of arginine at positions 554 and 651 replacing lysine in the other sequences.

\section{GRP78 protein levels}

Western blotting assessed GRP78 expression in ground squirrel tissues using a rabbit anti-GRP78 polyclonal antibody raised against rat GRP78 synthetic peptide (residue 645-654 at the C-terminal). On one-dimensional SDS-PAGE gels, all tissues showed a single band, corresponding to the known molecular mass (78 $\mathrm{kDa})$ of GRP78. The specificity of the GRP78 antibody was further confirmed with twodimensional electrophoresis which resulted in antibody cross-reaction with only a single spot on the gel (Fig. 2.6) corresponding with the known molecular mass and isoelectric point $(\mathrm{pI}=5.0$ ) of mouse GRP78 (Calvert et al., 2003). Fig. 2.7A shows representative Western blots of GRP78 protein levels in seven tissues of ground squirrels and Fig. 2.7B shows the mean ratio hibernating:euthermic in each tissue. During hibernation, GRP78 levels were significantly elevated in BAT and brain, by 1.57- and 1.37-fold, respectively, whereas protein content significantly decreased in heart and liver to 81 - and $58 \%$ of the euthermic value, respectively. The results for BAT and brain were consistent with the transcriptional up-regulation shown in Fig. 2.2B. 


\section{Binding of ATF4 to the ATF/CRE-like site of the grp78 promoter}

ATF4 is a transcription factor that is known to regulate grp78 expression in other systems. To determine whether ATF4 might also be involved in the hibernationresponsive expression of $g r p 78$, the interaction of ATF4 with the $g r p 78$ promoter was investigated in gel mobility shift assays using synthetic radiolabeled oligonucleotides containing the ATF/CRE site, the binding domain of ATF4. Fig. 2.8A shows that the ${ }^{32} \mathrm{P}$-labeled probe bound to three major protein complexes on the gel (I, II and III) as seen in lanes 2 and 3 . Competition experiments were used to determine which complex was specific for ATF4 binding. When unlabeled wild type probe was used as a competitor, ${ }^{32}$ P-labeling of complex I and II disappeared (lane 5). However, when mutant probe (containing a mutated ATF4 binding site) was used only complex II was out-competed (lane 4). From this we concluded that only complex I represented the specific ATF4/grp78 complex. None of the competitors inhibited the formation of complex III which was present in all the lanes. Furthermore, to confirm that complex I was specific to ATF4 binding, a supershift assay was performed with an antibody directed against a nuclear cofactor of ATF4, CREB-1. Previous studies have provided evidence that CREB-1 forms an in vivo complex with ATF4 to achieve the activation and regulation of genes (Luo et al., 2003). Anti-Bcl2 antibody was used to control for nonspecific binding in the supershift assay. Fig. 2.8B shows a shifted band of complex I (lane 3 and 4) at a position of slower mobility in the presence of antiCREB1 antibody. In contrast, no supershift was observed with anti-Bc12 antibody (lane 5 and 6). 


\section{DISCUSSION}

The accumulation of unfolded or misfolded proteins triggers the UPR, which then acts to release cells from the stress condition (Schröder and Kaufman, 2005; Patil and Walter, 2001). The UPR encompasses a number of complex signal transduction cascades, responsible for various cytoprotective measures that are undertaken when cells are experiencing ER stress (Schröder and Kaufman, 2005). GRP78 accumulation is an integral part of the UPR (Lee, 2001) and various lines of evidence indicate that GRP proteins have a neuroprotective role against excitotoxicity and apoptosis by suppressing oxidative stress and stabilizing calcium homeostasis (Lee et al., 2002). The up-regulation of GRP78 in two key organs, BAT and brain, during hibernation provides presumptive evidence that ground squirrel organs may undergo ER stress during hibernation. Gene expression was strongly increased with grp78 mRNA transcript levels being 3.5- and 4.1-fold higher, respectively, in BAT and brain of hibernators, compared with euthermic controls, whereas GRP78 protein levels also rose significantly by 1.57 - and 1.37 -fold, respectively. Interestingly, in a study on hibernating bats (Rhinolopus ferrumequinum), Lee et al. (2002) found that GRP78 protein levels increased by 1.7 -fold in bat brain within the first $30 \mathrm{~min}$ of arousal, compared with levels in the torpid state. Combined with our data, this may indicate that the elevation of grp 78 transcript levels that is initiated during entry into torpor supports GRP78 protein synthesis not only during torpor but also further enhances GRP78 production during arousal. This may be a valuable mechanism for ensuring that GRP78 chaperone levels are optimally elevated to deal with ER stress during arousal. ER stress may arise particularly from oxidative stress during arousal and other studies have shown that hibernators show adaptations of antioxidant defense mechanisms including have high constitutive activities of antioxidant enzymes and 
metabolites and up-regulation of selected antioxidant enzymes during torpor (Barja de Quiroga, 1992; Carey et al., 2000; Eddy et al., 2005b; Drew et al., 2002; Osborne and Hashimoto, 2006).

It is interesting that GRP78 up-regulation during hibernation was confined to two organs of ground squirrels that play essential roles in survival during hibernation, brain and BAT. Brain must maintain its sensing and signaling capacities over $\mathrm{Tb}$ values that can range from 0 to $37^{\circ} \mathrm{C}$. BAT is the key organ that is responsible for rewarming the animal back to euthermic $\mathrm{Tb}$ during arousal from torpor. BAT also provides low level heating of the body during torpor if ambient temperature drops below $0^{\circ} \mathrm{C}$. Hence, unlike most other tissues of hibernators, BAT maintains high metabolic activity during hibernation. Multiple hibernation-responsive proteins are synthesized and the ribosomal protein synthesis machinery is not suppressed in BAT during torpor as it is in other organs (Storey and Storey, 2004; Hittel and Storey, 2002). This allows adjustments to be made to the thermogenic capacity of the organ in response to ambient environmental conditions; for example, if ambient temperature in the burrows of squirrels falls below $0^{\circ} \mathrm{C}$, the thermogenic capacity of BAT is enhanced by upregulation and increased synthesis of the mitochondrial uncoupling protein 1 (UCP 1) (Boyer and Barnes, 1999). It is notable, therefore, that the two organs that are most critical to survival at low $\mathrm{Tb}$ during torpor are the ones that show GRP78 up-regulation during torpor - the brain that detects Tb (in the hypothalamus) and determines the timing of periodic arousals and the BAT that provides both low level heating to prevent freezing and high level thermogenesis to power arousal.

Previous work on GRP78 has shown that the protein sequence contains four evolutionarily-conserved hydrophobic domains that form transmembrane helices with domain I matching the leader peptide that targets GRP78 to the ER (Ramachandra et 
al., 2003; Wooden and Lee, 1992). Our results show that the ground squirrel GRP78 sequence, as compared with other mammalian sequences, has a substitution in the region equivalent to domain $\mathrm{I}$ at the $\mathrm{N}$-terminal; a valine residue (high hydrophobicity value: 4.2 ) at position 9 replacing methionine (low hydrophobicity value: 1.9 ) in the human sequence or alanine (low hydrophobicity value: 1.8 ) in the mouse and rat sequences. This may suggest a stronger anchoring of squirrel GRP78 in the ER. GRP78 resides in the ER lumen and performs its folding function via the carboxyterminus, coordinating the sequential binding and release of protein substrates (Gething, 1999). Indeed, the carboxyterminal of 13-lined ground squirrel GRP78 sequence contains the sequence RDEL at position 651 which matches the ER target sequence, the common retention signal for ER proteins (Pelham, 1990; Munro and Pelham, 1987), identified using the database of protein families and domains (PROSITE). Arginine (R) replaces the similar amino acid lysine that occurs in the signal sequence of the other mammalian species (Fig. 2.4). Moreover, it has been shown by Ramachandra et al. (2003) that in addition to the carboxyterminal function of GRP78, the N-terminus is within the cytoplasm and this interacts with cytosolic components via its ATP binding domain. At that N-terminus, GRP78 plays an antiapoptotic function by interacting with caspase- 7 and caspase- 12 both in vivo and in vitro to block caspase activation. This may also be an important feature of GRP78 action in 13-lined ground squirrel organs - the inhibition of apoptosis that would normally be triggered by hypothermia or oxidative stresses in nonhibernating species. The four unique amino acid substitutions in ground squirrel GRP78 as compared with nonhibernating mammals may also help to maintain optimal GRP78 function in the ER over the wide range of $\mathrm{Tb}$ values $\left(\sim 0^{\circ} \mathrm{C}\right.$ to $\left.37^{\circ} \mathrm{C}\right)$ experienced by hibernators. Temperature has major effects on protein conformation and protein-protein binding 
interactions and selected substitutions in the GRP78 sequence may ensure that the important function of GRP78 as a chaperone is unimpaired in organs during cold torpor. Other proteins that are typically highly conserved in mammals, such as fatty acid binding protein (the intracellular lipid transporter), also show selected amino acids substitutions in hibernator sequences that appear to modify protein conformation for enhanced protein function at low $\mathrm{Tb}$ (Hittel and Storey, 2001; Eddy and Storey, 2004).

A large amount of work has established that, in addition to its folding function and its ability to block the apoptotic process, GRP78 binds to three transducers of ER stress signaling to maintain them in an inactive form: the activating transcription factor 6 (ATF6), the serine/threonine endoribonuclease IRE1, and the serine/threonine kinase PERK (Schröder and Kaufman, 2005; Rutkowski and Kaufman, 2004; Lee, 2005). These sensors are activated when they are released from GRP78 under ER stress. The resulting effects of their activation include an up-regulation of genes encoding proteins involved in the secretory pathways (ER resident chaperones), proteins involved in ER-associated protein degradation and a shut down of overall protein synthesis. In addition, it has been reported that under ER stress, the pathway that is activated most rapidly is translational repression mediated by PERK which phosphorylates its direct substrate, the $\alpha$ subunit of the eukaryotic initiation factor 2 (eIF2 $\alpha$ ) (Rutkowski and Kaufman, 2004). Studies by our lab and others have shown that during hibernation, $\operatorname{eIF} 2 \alpha$ is phosphorylated to block the delivery of the initiating Met-tRNA to the ribosomal preinitiation complex (Storey and Storey, 2004, Hittel and Storey, 2002; Frerichs et al., 1998). Furthermore, the phosphorylation of eIF2 $\alpha$ was found to induce the translation of ATF4 which has been shown to bind to its response element in the promoter of several genes involved in the UPR including 
grp78 (Luo et al., 2003). ATF4 is known to bind to the ATF/CRE-like site of the grp 78 promoter to activate its transcription in response to hypoxia and other ER stress (such as those induced by thapsigargin and tunicamycin) (Luo et al., 2003). Hence, we examined whether ATF4 could also regulate grp78 in ground squirrels. Electrophoretic mobility shift and supershift assays demonstrated that ATF4 from ground squirrel can bind to the ATF/CRE-like site in the promoter region of $g r p 78$. Hence, ATF4 could be the transcription factor responsible for grp78 up-regulation during hibernation.

The supershift assay made use of a functional interaction with a previously established DNA binding domain at the CRE/ATF site designed from the rat grp78 promoter (Luo et al., 2003; Alexandre et al., 1991). Fig. 2.8B (lanes 3 and 4) provides evidence that complex I is specific to ATF4 since the complex I band shifted to a position of slower mobility in the presence of the anti-CREB1 antibody in the supershift assay. Complex II and complex III were determined to be nonspecific signals created by the binding of ubiquitous factors that have general affinity for DNA and that are present in high abundance in cell extracts, as previously reported (Alexandre et al., 1991).

Overall, these data show a coordinated increase in both grp78 mRNA and GRP78 protein levels in BAT and brain of hibernating squirrels compared to euthermic controls and this appears to be physiologically relevant to survival during hibernation. The up-regulation of GRP78 upon ER stress suggests its pivotal role in correctly folding hibernation-responsive proteins, maintaining cellular homeostasis, balancing between cell survival and apoptosis, and suggests an important protective response used by cells when they enter the hypometabolic, low $\mathrm{T}_{\mathrm{b}}$ state of hibernation. The activation of grp78 expression during hibernation may be mediated 
by ATF4 although other transcription factors including ATF6 and IRE1/XBP1 can also regulate the gene. Studies have only recently begun to identify the transcription factors involved in hibernation-responsive gene expression and much more work remains to be done. To date, for example, the up-regulation of some proteins/enzyme involved in lipid catabolism and mitochondrial energy production have been linked with elevated levels of PPAR $\gamma$ and PGC-1 in hibernator organs (Eddy and Storey, 2004; Eddy et al., 2005a). 
Fig. 2.1: Model of GRP78 role: central regulator for ER stress signaling and survival. Upon ER stress triggered by the accumulation of malfolded protein in the ER lumen, GRP78 is released from the sensors ATF6, IRE1 and PERK which activate the UPR. GRP78 is also in complex with procaspases that associate with the ER membrane, therefore regulating the balance between cell survival and apoptosis in ER-stressed cells. Taken from Lee (2005). 
Fig. 2.1:

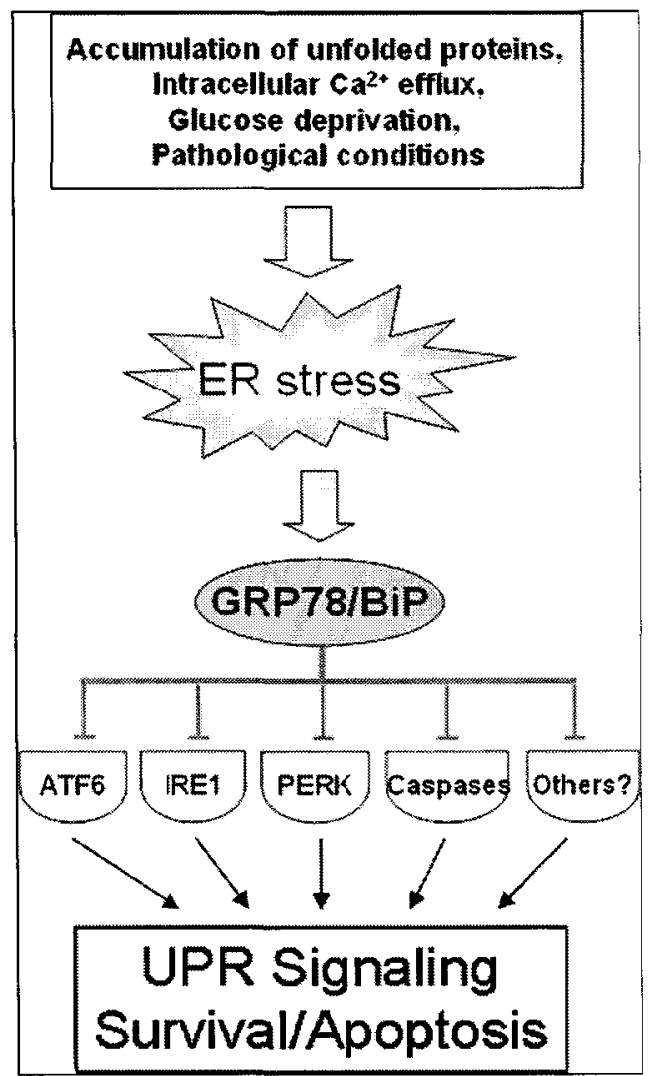


Fig. 2.2: Effect of hibernation on grp78 mRNA levels in seven tissues of thirteen-lined ground squirrels. (A) Representative RT-PCR product bands amplified from mRNA samples from tissues of euthermic and hibernating squirrels. (B) Histogram showing the mean ratio of normalized PCR product levels (relative to $\alpha$-tubulin) in tissues from hibernating versus euthermic ground squirrels. The highest dilutions for $g r p 78\left(10^{-4}\right.$ or $10^{-}$ ${ }^{5}$ ) and $\alpha$-tubulin (from the same sample tube) which gave visible PCR product bands were chosen for quantification. Data are means \pm S.E.M., $n=3$ independent trials. BAT is brown adipose tissue; muscle is hind leg skeletal muscle; $\mathrm{E}$ - euthermic, $\mathrm{H}$ - hibernating. * - Value for hibernating sample is significantly different from the corresponding euthermic value, $\mathrm{P}<0.05$. 
Fig. 2.2:

A.

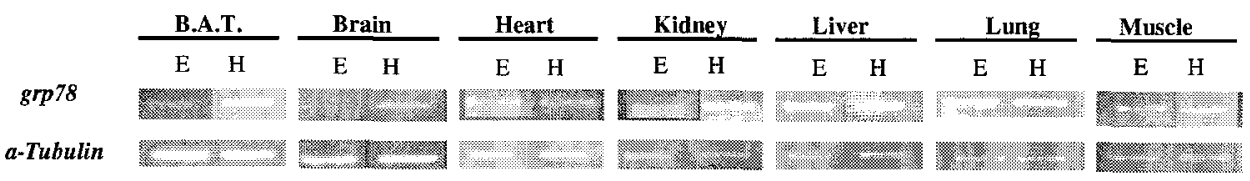

B.

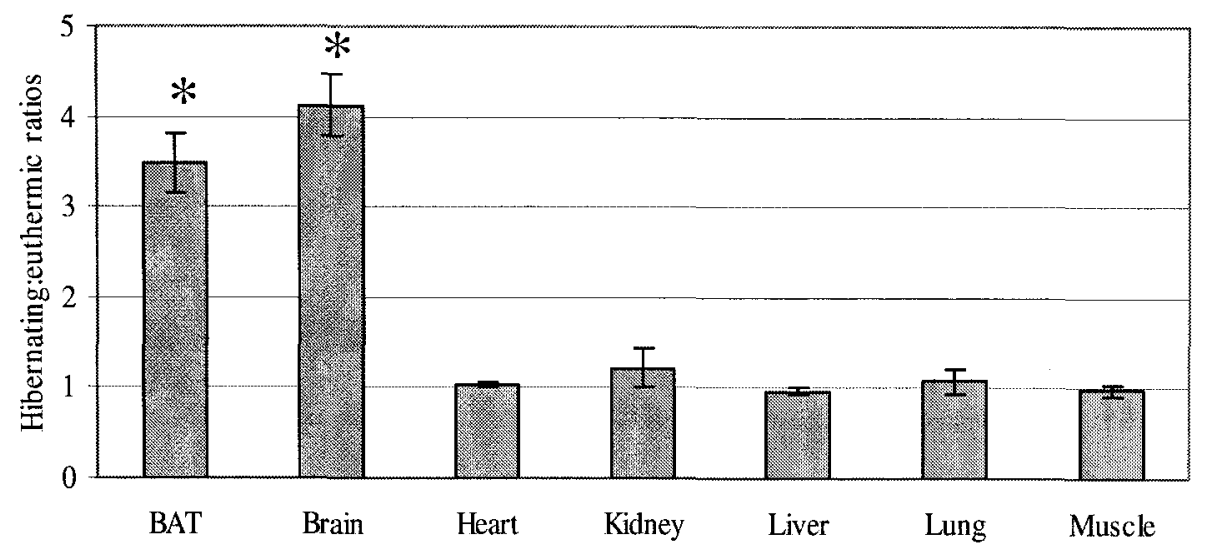


Fig. 2.3: Complete cDNA sequence of grp78 amplified from brown adipose tissue of thirteen-lined ground squirrels (S. tridecemlineatus) along with the translated amino acid sequence. Nucleotides and amino acids are numbered on the left. A single open reading frame was predicted from the nucleotide sequence and encoded a polypeptide with 654 residues. The start and stop codon are underlined in the cDNA sequence. 
Fig. 2.3:

TTCCCTGCCTGCTGACCGAGAGCAAGTGGCCGACAGCTACGGAAGATGAAGCTCTCTCTG

GTGGCCGCGGTGCTGCTGCTGCICTGCGCGGCGCGGGCCGAGGAGGAGGACAAGAAGGAG 


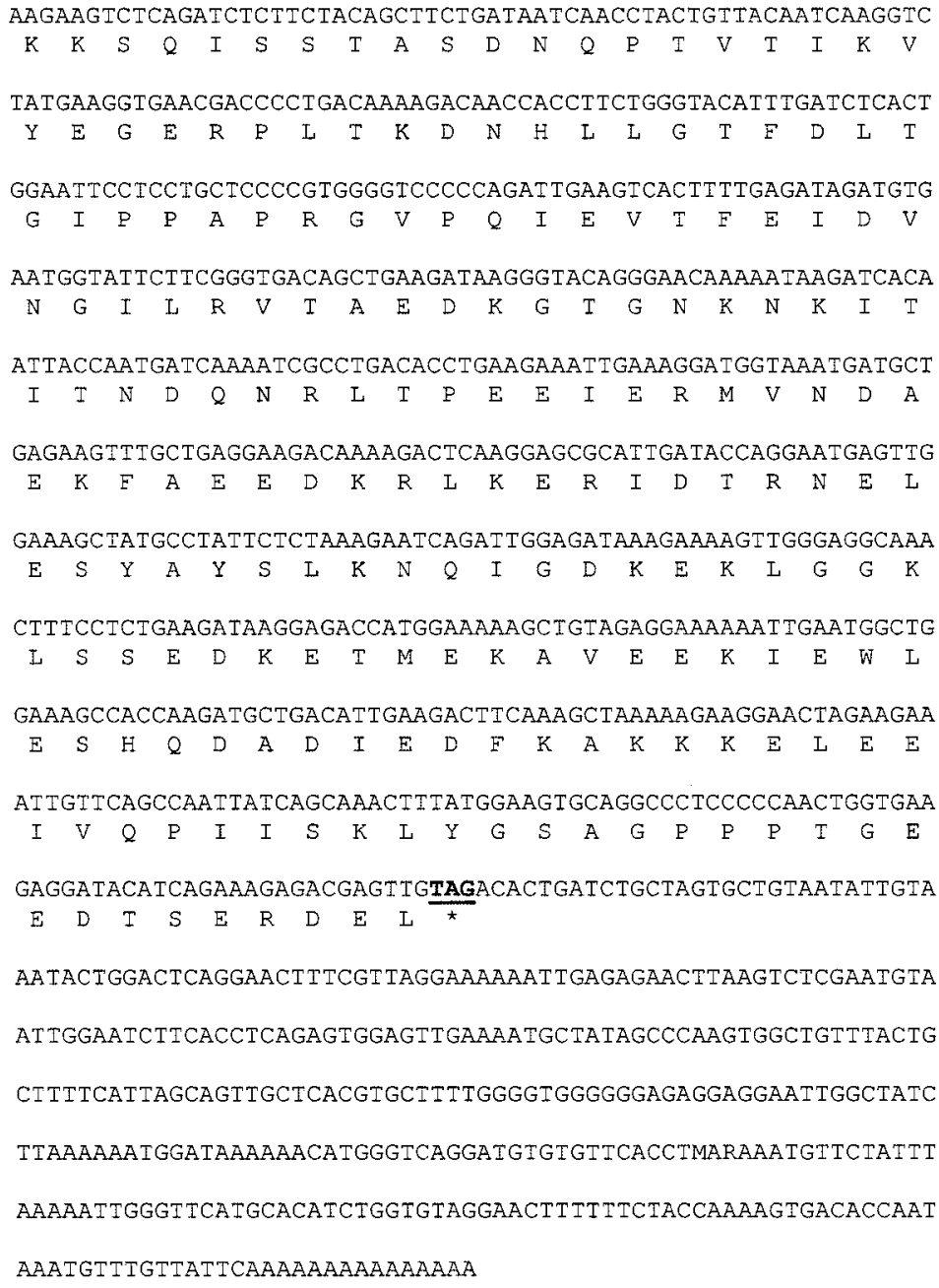


Fig. 2.4: Ground squirrel (Spermophilus tridecemlineatus) GRP78 full length amino acid sequence (GenBank accession number DQ166628) aligned with human (Homo sapiens), mouse (Mus musculus) and rat (Rattus norvegicus) sequences (Genbank accession numbers NP_005338, NP_071705 and NP_037215, respectively). Unique amino acid substitutions in the squirrel sequence are indicated in bold underline. Dashes (-) represent amino acids in the human, mouse or rat sequences that are identical with the squirrel sequence. Periods are present in the alignment to indicate where an amino acid is not present in the coding region of one of the species. 
Fig. 2.4:

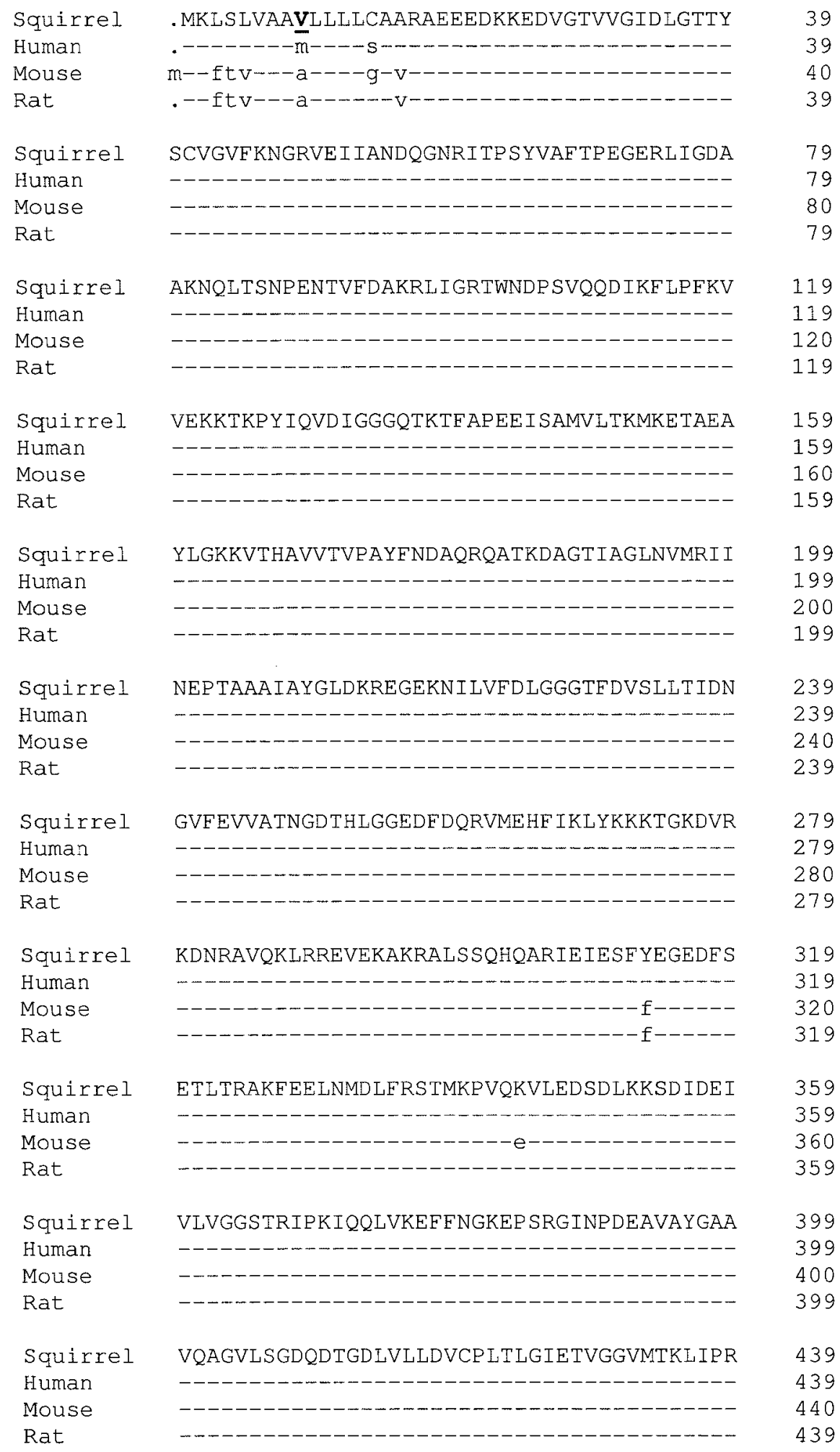




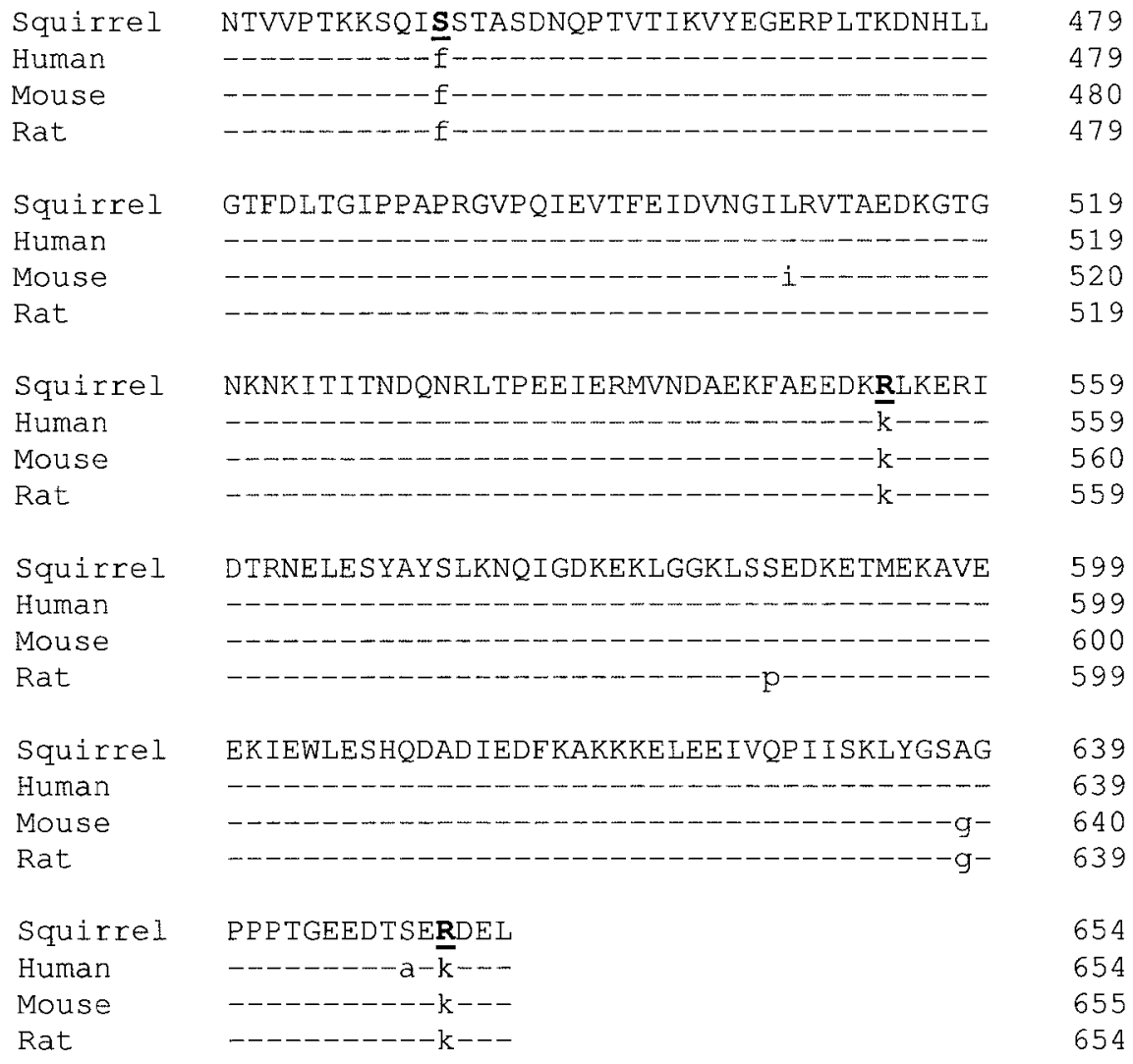


Fig. 2.5: (A) Cladogram prepared with DNAMAN showing the percent identities between the protein sequences of GRP78 (654 amino acids) from ground squirrel ( $S$. tridecemlineatus), human, mouse and rat (accession numbers as in Fig. 2.4). (B) Homology matrix showing amino acid identity of the thirteen-lined ground squirrel GRP78 sequence compared with GRP78 protein of other mammalian species: human, mouse and rat. 
Fig. 2.5:

A. GRP78 protein cladogram

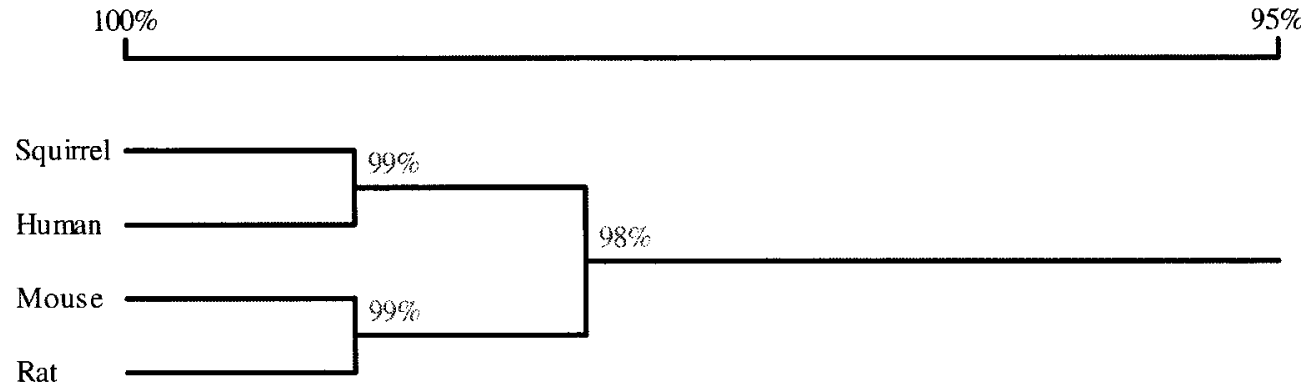

B. Homology matrix of GRP78 protein sequences

$\begin{array}{lll}\text { Squirrel } & 100 \% & \\ \text { Human } & 99.1 \% 100 \% & \\ \text { Mouse } & 98.0 \% 98.3 \% 100 \% \\ \text { Rat } & 98.3 \% 98.5 \% 99.4 \% 100 \%\end{array}$


Fig. 2.6: Representative 2D-PAGE Western blot of muscle extract from euthermic (control) thirteen-lined ground squirrel immunoblotted with anti-GRP78 antibody. The gel was loaded $10 \mu \mathrm{g}$ of soluble protein. Separation in the first dimension was via isoelectrofocusing and the second dimension was SDS-PAGE; proteins were transferred to PVDF membranes before immunoblotting with anti-GRP78 antibodies. The crossreacting spot is circled and corresponds to a pI of 5.0 and a molecular weight of $\sim 78 \mathrm{kDa}$, consistent with GRP78 from other sources. 
Fig. 2.6:

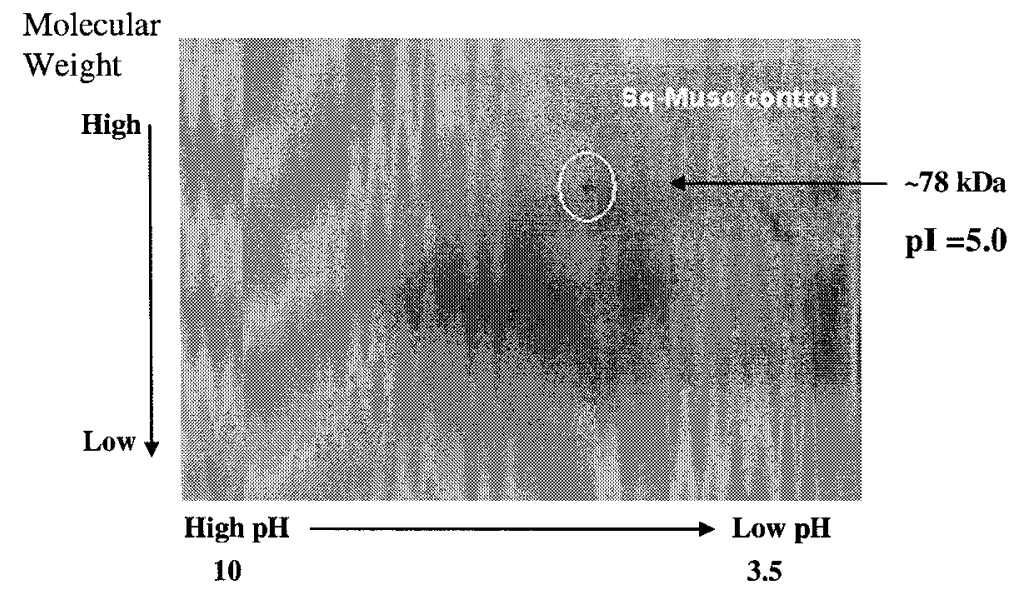


Fig. 2.7: (A) Representative Western blots showing GRP78 protein levels in seven tissues from euthermic $(\mathrm{E})$ and hibernating $(\mathrm{H})$ ground squirrels. (B) Histogram showing the relative levels of GRP78 protein in tissues from hibernating versus euthermic animals. Data are means \pm S.E.M., n= 3-5 independent trials. * - Mean value for hibernating sample is significantly different from the corresponding euthermic value, $\mathrm{P}<$ 0.05 . 
Fig. 2.7:

A.

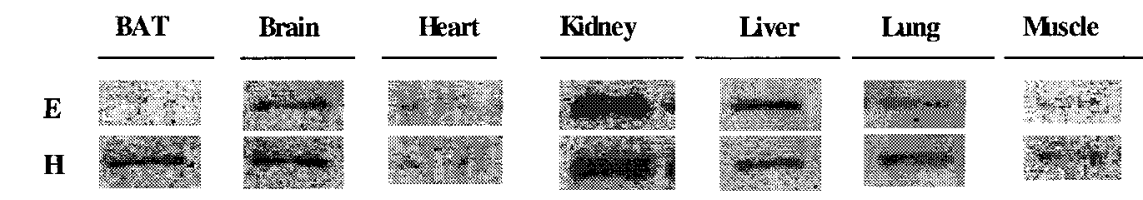

B.

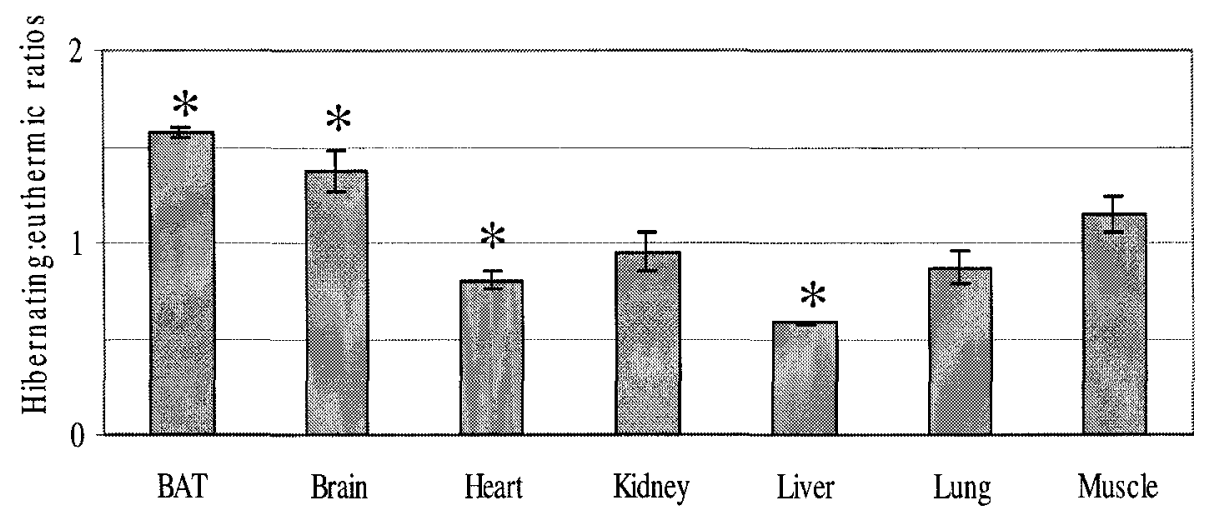


Fig. 2.8: Electrophoretic mobility shift and supershift assays using nuclear extracts from brain of 13-lined ground squirrels. The ATF/CRE probe was designed from the rat $g r p 78$ promoter and contains the binding domain for the transcription factor ATF4. (A) Electrophoretic mobility shift: Lane 1, free probe; Lanes 2 and 3, nuclear extracts from hibernating and euthermic animals, respectively, with no competitor; Lanes 4 and 5, nuclear extracts from hibernating squirrels preincubated with competitors before adding the labeled probe (lane 4: mutant probe for nonspecific competition, lane 5: wild type unlabeled probe for specific competition). (B) Gel mobility shift and supershift assays. Lanes 1 and 2, nuclear extract from hibernating squirrels incubated with unlabeled wild type probe and no competitor, respectively. In Lanes 3 and 5 (hibernating nuclear extract) and Lanes 4 and 6 (euthermic nuclear extract) $1 \mu \mathrm{g}$ of anti-CREB1 antibody (lanes 3 and 4) or anti-Bcl2 antibody (lanes 5 and 6) were added and incubated for $20 \mathrm{~min}$ before adding labeled probe. 
Fig. 2.8:

A.

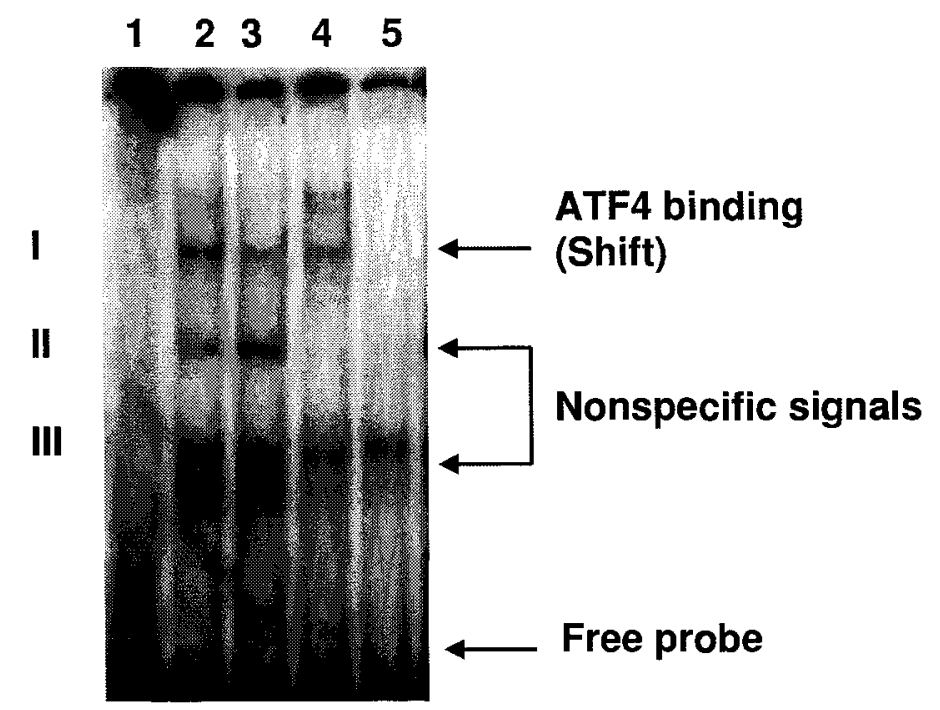

B.

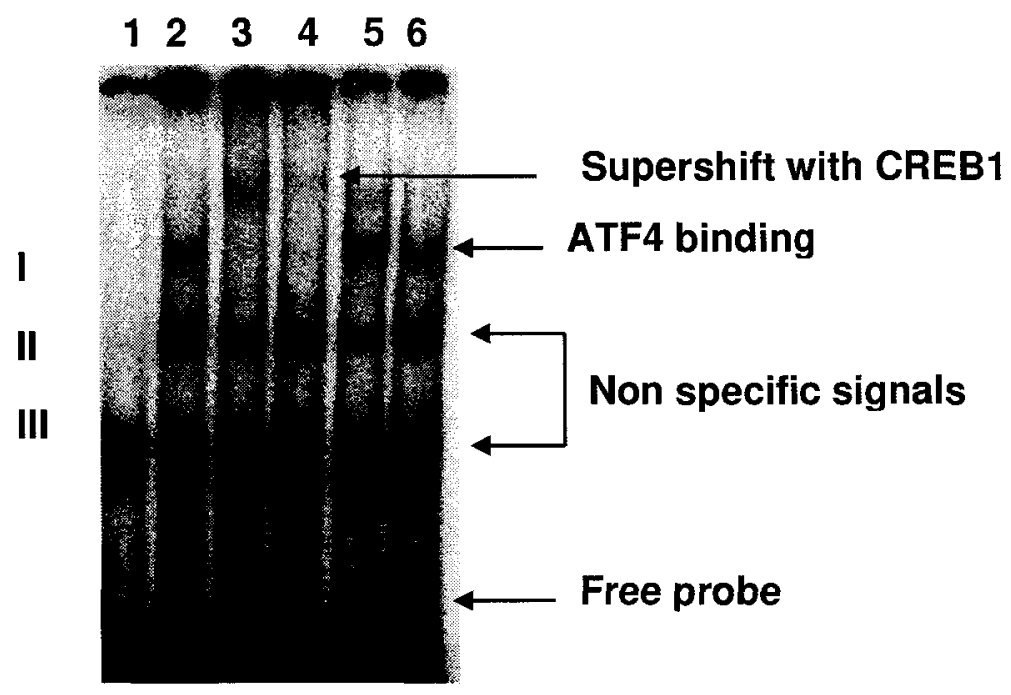




\title{
Chapter 3
}

\section{Coping with the stress: expression of ATF4, ATF6 and downstream targets in hibernating ground}

\author{
squirrel tissues
}




\section{INTRODUCTION}

A variety of physiological stresses and pathologies have in common the accumulation of misfolded proteins. This is collectively called endoplasmic reticulum (ER) stress and elicits the activation of a complex signal transduction cascade known as the $\underline{\text { Unfolded Protein }}$ Response (UPR) as well as the ER associated protein degradation (ERAD) pathway (Schröder and Kaufman, 2005). Conditions that can invoke the UPR include inhibition of N-linked glycosylation in the ER, depletion of ER calcium stores, reductive stress, hypothermia, ischemia, hypoxia and oxidative stress (Rutkowski and Kaufman, 2004). Mammalian cells respond to ER stress by attenuating translation and activating the UPR. The UPR coordinates a program of modified gene transcription and protein translation that includes the preferential up-regulation of genes encoding ERresident chaperones, factors that regulate the metabolism and redox environment of the cell, and proteins involved in the ERAD pathway (Rutkowski and Kaufman, 2004). Given that hibernators naturally endure conditions that would elicit ER stress in nonhibernating mammals, we wondered how hibernators dealt with these potential problems and whether the UPR was naturally activated or suppressed during torpor. A model of UPR signaling in mammalian cells investigated in this study is summarized in Fig. 3.1. GRP78, one of the most abundant chaperones residing in the ER, is a central regulator of the ER stress response (Lee, 2005). The data in Chapter 2 showed that grp78 transcript levels were strongly elevated by $3.5-4.1$ fold in brown adipose tissue and brain of hibernating 13-lined ground squirrels (Spermophilus tridecemlineatus), as compared with euthermic animals. Furthermore, GRP78 protein was also 1.4-1.6 fold higher in these organs during torpor. Hence, I had initial evidence that ER stress and the UPR may 
be a component of hibernation.

GRP78 regulates the ER stress response via its ability to control the activation of three transmembrane ER stress sensors through a binding-release mechanism: inositolrequiring kinase 1 (IRE1), PKR-like endoplasmic reticulum kinase (PERK), and activating transcription factor 6 (ATF6) (Lee, 2005). Upon ER stress, these signaling molecules dissociate from GRP78 and undergo posttranslational modifications that activate them. Phosphorylated PERK (pPERK) triggers inhibition of ribosomal protein translation via phosphorylation of the $\alpha$-subunit of the eukaryotic initiation factor- $2 \alpha$ $(\mathrm{eIF} 2 \alpha)$ to decrease the amount of client protein entering the ER (Jiang and Wek, 2005; Harding et al., 2000) whereas activation of IRE1 and ATF6 increases transcription of genes encoding ER chaperones and proteins involved in apoptosis (Cullinan and Diehl, 2006; Rutkowski and Kaufman 2004). In response to stress, inactive $90 \mathrm{kDa}$ ATF6 translocates from the ER to the Golgi where it is processed to its active $50 \mathrm{kDa}$ form via sequential cleavage by site- 1 and site-2 proteases (S1P and S2P). The active ATF6-50 $\mathrm{kDa}$ then moves into the nucleus, forms complexes with co-activators, and binds to the ER stress-response elements (ERSE) in genes such as grp78, grp94, PDI and CHOP to induce their transcriptional activation (Okada et al., 2002).

Multiple studies have reported that phosphorylation of eIF2 $\alpha$ enhances expression of the activating transcription factor $4(\mathrm{ATF} 4)$, a member of the activating transcription factor/cAMP response element binding proteins (ATF/CREB) family that mediates diverse transcriptional regulatory effects (Jiang and Wek, 2005; Luo et al., 2003; Blais et al., 2004). In turn, ATF4 activates both pro-survival and pro-apoptotic genes that participate in the UPR. For example, ATF4 forms a complex with its nuclear co-activator, 
the phosphorylated form of CREB1, to bind to the response element, the ATF/CRE-like site in the promoter region and activate the pro-survival gene grp78 (Luo et al., 2003; Mamady and Storey, 2006). One of the pro-apoptotic genes that is downstream of ATF4 is the growth arrest and DNA damage-inducible protein 153 (GADD153), also known as CHOP, a member of the CCAAT/Enhancer Binding Protein homologous (C/EBP) family of transcription factors. CHOP is expressed in response to a number of stresses including impaired protein folding in the ER, reactive oxygen species, hypoxia, and nutrient deprivation (Wang et al., 1996). Another downstream gene regulated by ATF4 is GADD34 which was recently identified as the factor that associates with protein phosphatase 1 (PP1) to dephosphorylate phospho-eIF2 $\alpha$ in a feedback regulatory loop that promotes translational recovery (Ma and Hendershot, 2003).

I hypothesized that the UPR would be activated during torpor to provide proactive protection to cells to preserve and stabilize cellular proteins and the protein synthesizing machinery over long periods of cold torpor. The present chapter examines the expression of ATF4, a central regulator of the UPR, at both the mRNA and protein levels in organs of $S$. tridecemlineatus in order to evaluate its role in hibernation. The responses of a variety of proteins involved in the UPR including pPERK, ATF6 (50 kDa), CREB-1, pCREB1, CHOP and GADD34 are also evaluated. The data indicate that ATF4 is induced at the translational level during torpor and moves into the nucleus where, in complex with greatly elevated levels of its co-activator p-CREB1, it mediates an integrated stress response to protect cells during torpor. 


\section{MATERIAL AND METHODS}

Animals

Thirteen-lined ground squirrels (Spermophilus tridecemlineatus) were prepared and treated as described in Chapter 2.

\section{Total RNA extraction and RT-PCR and 3'RACE}

Total RNA was isolated from tissue samples using Trizol ${ }^{\mathrm{TM}}$ reagent (Invitrogen) and the quality was assessed as described in Chapter 2. cDNA was reverse transcribed, and serially diluted, then atf4 was amplified using RT-PCR. The reaction conditions were as follows: $94^{\circ} \mathrm{C}$ pre-heat for $3 \mathrm{~min}$, followed by 35 cycles of $95^{\circ} \mathrm{C}$ for $30 \mathrm{sec}$ (denaturation), $57^{\circ} \mathrm{C}$ for $30 \mathrm{sec}$ (annealing) and $72^{\circ} \mathrm{C}$ for $30 \mathrm{sec}$ (elongation), then a final step of $7 \mathrm{~min}$ of $72^{\circ} \mathrm{C}$ (final elongation). Primers for atf 4 were designed based on the human atf4 sequence (GenBank accession number NM_182810) and according to a consensus sequence of atf 4 from other mammalian species. The nucleotide sequences of the atf4 primer pair were 5'-GGAGGTGGCCAAGCACTTCA-3' and 5'CTTCTGGCGGTACCTAGTGG-3'. The housekeeping gene used as an internal control for PCR was $\alpha$-tubulin as described in Chapter 2. The RT-PCR of atf4 generated a product of 781 bp that was sequenced by CORTEC (Kingston, ON) and verified as encoding atf4 using the program BLASTN at the NIH. The 3' end was amplified as previously described in Chapter 2, using a Takara 3' RACE kit (Promega). The gene specific primer (GSP) used was: 5' -AACCATGCCAGATGAGCTTT- 3'. The 3'RACE generated a fragment of $889 \mathrm{bp}$, verified to encode the expected atf4 gene. The processed and assembled sequence was submitted to Genbank (accession number DQ324000). The 
deduced amino acid sequence was also verified to be ATF4 via the BLASTP program.

\section{Western blotting}

Protein extracts were prepared from tissue samples of euthermic and hibernating ground squirrels. Equal amounts of protein $(10-20 \mu \mathrm{g})$ were loaded into each lane of 8 or $12 \%$ SDS polyacrylamide gels, electrophoresed and then electroblotted onto polyvinylidene difluoride (PVDF) membranes as described in Chapter 2. After the transfer of proteins, membranes were blocked for 30-60 min in Tris buffered saline containing Tween-20 (TBST: $20 \mathrm{mM}$ Tris base, $140 \mathrm{mM} \mathrm{NaCl}, 0.1 \%$ v/v Tween-20) with $2.5 \%$ non-fat dried milk added. The blots were then incubated at $4{ }^{\circ} \mathrm{C}$ overnight with a primary antibody diluted in TBST. The antibodies used were rabbit polyclonal antibodies against ATF4 diluted 1:500 and goat polyclonal antibodies against ATF6 diluted 1:100 (both from Santa Cruz Biotechnology Inc.), rabbit polyclonal antibodies recognizing the phosphorylated form of PERK diluted 1:1000 (Cell Signaling), rabbit polyclonal antibodies against CREB-1 and phospho-CREB-1 diluted 1:250 (both from Santa Cruz Biotechnology Inc.), mouse monoclonal antibodies against GADD153 (CHOP) diluted 1:1000 (Stressgen), and rabbit antibodies against GADD34 diluted 1:300 (Santa Cruz Biotechnology Inc.). After incubation, blots were washed several times with TBST and then incubated at room temperature for $2 \mathrm{~h}$ with a secondary antibody, anti-rabbit and anti-mouse (Cell Signaling) or anti-goat (Santa Cruz Biotechnology Inc.) IgG conjugated to horseradish peroxidase at a 1:2000 dilution. Detection of signal on the PVDF membrane was done using an enhanced chemiluminescence (ECL) system as recommended by the manufacturer (Pierce). The membrane was scanned using the 
ChemiGenius (Syngene, MD, USA) and the resulting image was analyzed with the associated Gene Tools software.

\section{Preparation of cytoplasmic and nuclear extracts}

Cytoplasmic and nuclear extracts from brain, heart and muscle of euthermic and hibernating ground squirrels were prepared as described in Chapter 2, using a slight modification of the method described by Dignam et al. (1983). Protein concentration in each fraction was determined and aliquots containing 10-20 $\mu \mathrm{g}$ were mixed with $2 \mathrm{X}$ SDS-PAGE sample buffer, loaded onto 10\% SDS-PAGE minigels and separated at $180 \mathrm{~V}$ for $45 \mathrm{~min}$. After transfer onto PVDF membranes, immunoblotting was conducted as described above. Western blotting with anti-histone $\mathrm{H} 3$ antibody was performed as described in chapter 2 , to confirm the integrity of the nuclei when separated from the cytoplasmic fraction; histone $\mathrm{H} 3$ band was found in the nuclear extracts and not in the cytoplasmic fraction. In addition, the quality of cytoplasmic fraction was verified by immunoblotting with pCREB-1 antibody; pCREB1 band was found in the cytoplasmic fraction and not in the nuclear fraction (data not shown).

\section{Statistics}

Western blot band intensities were normalized against three Coomassie stained protein bands that did not show variations between euthermic and hibernating states. RTPCR bands of atf 4 were normalized against band intensities for $\alpha$-tubulin amplified from the same cDNA reaction. Statistical testing for differences between normalized band intensities from euthermic versus hibernating samples used the Student's $t$-test with 
significance levels of $\mathrm{P}<0.05$ or $\mathrm{P}<0.01$. The mean ratios of hibernating to euthermic values and the S.E.M on the ratios were then calculated; the data are shown as mean ratios \pm S.E.M in histograms. All data result from a minimum of $n=3$ independent isolations from ground squirrel tissues.

\section{RESULTS}

\section{PERK and ATF6 protein expression}

Proximal events for UPR activation include the activation of ER-resident signaling molecules such as PERK and ATF6 in order to initiate transcriptional and translational programs that alleviate the ER stress. To investigate how these systems respond in hibernation, protein levels of p-PERK and ATF6 were assessed in tissues of euthermic versus hibernating squirrels. Experiments using the p-PERK antibody with protein extracts from brain and kidney (data not shown) showed cross-reaction with a single protein band of $\sim 170 \mathrm{kDa}$ in both euthermic and hibernating states (Fig. 3.2A). However, densitometry analysis showed no difference in p-PERK levels in brain between the two states. Analysis of pPERK in kidney also revealed unchanged level between the two states (data not shown). Experiments using anti-ATF6 antibodies detected the $50 \mathrm{kDa}$ protein in various tissues of squirrels but no significant difference in expression occurred in brain or heart of hibernating squirrels compared to euthermia (Fig. 3.2B). However, a significantly higher level of ATF6 expression was found in skeletal muscle of hibernating squirrels, $1.20 \pm 0.01$ fold higher $(\mathrm{P}<0.05)$ compared to euthermic animals. 


\section{Effect of hibernation on ATF4 protein expression}

Fig. 3.3A shows the protein levels of ATF4 in six tissues of euthermic and hibernating ground squirrels. Western blot analysis showed a cross-reacting band at $\sim 40$ $\mathrm{kDa}$, consistent with the known molecular weight of mammalian ATF4. ATF4 protein levels increased significantly during hibernation in BAT, brain and skeletal muscle by $2.51 \pm 0.09,1.88 \pm 0.05$, and $2.48 \pm 0.09$ fold, respectively (Fig. 3.3B). By comparison, ATF4 levels were reduced during hibernation in heart to $56 \pm 1 \%$ of the control level. Levels of ATF4 were unaffected in kidney and liver.

\section{Atf4 amplification and transcript levels}

Using forward and reverse primers designed based on the human atf4 sequence (Genbank accession number NM_182810) but with a consensus sequence of atf4 from mouse and rat (BC085169 and NM_024403, respectively), a 781 bp cDNA was amplified from BAT of ground squirrels. After nucleotide sequencing, the cDNA was shown to encode $A T F 4$. Furthermore, an atf4 GSP was designed from the 781 bp partial atf4 cDNA sequence and used in $3^{\prime}$ RACE to extend and amplify the $3^{\prime}$ end of the ground squirrel atf4 sequence. The assembled ground squirrel atf4 cDNA sequence of $1032 \mathrm{bp}$ encoded a partial amino acid sequence (313 residues), including the $\mathrm{C}$ terminus (Fig. 3.4); this corresponded to approximately $89 \%$ of the full ATF4 sequence from human (351 amino acids). The ground squirrel atf4 cDNA sequence was submitted to GenBank with accession number DQ324000. Fig. 3.5 shows the partial ground squirrel ATF4 sequence aligned with the amino acid sequences of human, mouse and rat ATF4. The ground squirrel ATF4 sequence contained 19 unique amino acid substitutions that replaced 
amino acids that were conserved in all three nonhibernating mammals; these residues are shown in bold underline in Fig. 3.4. Among these were an isoleucine residue substituted at position 311 compared with lysine in the other species, and a glycine substitution at position 350 replacing a valine in the other sequences. The homology tree and homology matrix show that amino acid sequence of ground squirrel ATF4 was 85.9\%, 81.9\% and 83.1\% identical to the ATF4 amino acid sequence of human, mouse and rat respectively, over the amplified region (Fig. 3.6).

In order to determine whether the increase in ATF4 protein levels observed during hibernation resulted from increased gene expression, atf 4 mRNA transcript levels were quantified in six tissues from euthermic versus hibernating ground squirrels using relative RT-PCR that involved amplifying an internal control, $\alpha$-tubulin, simultaneously with the gene of interest. Fig. 3.7A shows representative bands for atf4 mRNA expression in each organ together with expression levels of $\alpha$-tubulin which were previously shown to be unchanged in ground squirrel tissues between euthermic and hibernating states. Transcript levels of atf 4 were normalized against $\alpha$-tubulin transcripts levels amplified from the same samples and mean normalized expression ratios in hibernation versus euthermia are shown in Fig. 3.7B. Transcript levels of atf4 increased significantly by 2.32 \pm 0.16 -fold $(\mathrm{P}<0.01)$ in liver during hibernation but decreased significantly to just $52 \pm 2$ $\%(\mathrm{P}<0.01)$ of the euthermic value in heart of hibernating squirrels. Transcript levels remained unchanged in all other tissues.

\section{Effect of hibernation on CREB1 and p-CREB1 protein expression}

Since ATF4 protein levels were up-regulated in selected tissues (BAT, brain, muscle) 
of hibernating ground squirrels, I next evaluated levels of its nuclear co-factor CREB1, a member of the ATF/CREB family that forms an in vivo complex with ATF4 upon ER stress. The CREB1 antibody cross-reacted with a single band of $\sim 43 \mathrm{kDa}$ in ground squirrel extracts, reflecting the known molecular weight of the mammalian protein. Fig. 3.8A shows Western blots of total CREB1 protein and levels of the phosphorylated (Ser133), active protein. Total protein levels did not change during torpor in four tissues but fell somewhat in kidney and muscle of hibernating squirrels to $87 \%$ and $52 \%$ of the euthermic values, respectively (data not shown). By contrast, levels of pCREB 1 increased significantly in all six organs of hibernating squirrels, particularly in brain and kidney where the ratio hibernating vs. euthermic was $7.44 \pm 0.22(\mathrm{P}<0.01)$ and $15.8 \pm 0.5(\mathrm{P}<$ 0.01), respectively (Fig. 3.8B). The hibernating:euthermic ratio was $4.28 \pm 0.19(\mathrm{P}<0.05)$, $3.58 \pm 0.14(\mathrm{P}<0.05), 2.07 \pm 0.05(\mathrm{P}<0.01)$ and $2.57 \pm 0.09(\mathrm{P}<0.01)$ for BAT, heart, liver and muscle, respectively.

\section{Distribution of ATF6, ATF4 and p-CREB1 in subcellular fractions of tissues}

To determine whether the transcription factors moved into the nucleus during hibernation, nuclear and cytoplasmic fractions were isolated from brain, heart, and skeletal muscle and the distribution of the three transcription factors was assessed by Western blotting. Fig. 3.9A shows representative Western blots for the $50 \mathrm{kDa}$ ATF6 protein in subcellular fractions of brain, heart and muscle. In heart, the amount of ATF6 in the nuclear fraction increased by 1.45 -fold $(\mathrm{P}<0.05)$ during hibernation as compared with euthermia (Fig. 3.9B). However, the distribution of ATF6 in brain was unaltered between euthermic and hibernating samples and in skeletal muscle levels of ATF6 in the 
nucleus remained unchanged during torpor but significantly increased by 1.47 -fold $(\mathrm{P}<$ $0.05)$ in the cytoplasm.

Fig. 3.10 shows the subcellular distribution of ATF4 and p-CREB1 in cytoplasmic and nuclear fractions of two organs, brain and muscle. ATF4 protein increased significantly during hibernation in both cytoplasmic and nuclear fractions from brain (by 1.46 fold and 1.17 fold, respectively, $\mathrm{P}<0.01$ ) and muscle (by 1.45 fold and 1.22 fold, respectively, $\mathrm{P}<0.05$ ) (Fig. 3.10A). Very large increases in $\mathrm{p}$-CREB1 occurred in both fractions during torpor, particularly in the nuclear fraction (Fig. 3.10B). Levels were significantly higher during hibernation in both cytoplasmic and nuclear fractions from brain (by 7.66-fold and 38.6-fold, respectively, $\mathrm{P}<0.01$ ) and skeletal muscle (by 3.80 fold and 25.2 fold respectively, $\mathrm{P}<0.05$ ).

\section{CHOP and GADD34 protein levels}

Protein levels of the transcription factors CHOP (GADD153) and GADD34 were also assessed via Western blotting in three tissues of euthermic and hibernating ground squirrels. Fig. 3.11 A shows that the protein content of CHOP responded differentially during hibernation in brain, heart and muscle. CHOP increased significantly by 1.25 -fold in heart during hibernation but decreased significantly to $61 \%$ of the euthermic value in muscle and was unchanged in brain. The rabbit polyclonal antibody raised against GADD34 crossreacted with a single protein band of $\sim 82 \mathrm{kDa}$ that corresponded with the

known size of the GADD34 protein in other mammals. During hibernation, levels of GADD34 protein were unchanged in muscle but decreased significantly to $77 \%(\mathrm{P}<$ $0.05)$ in brain and $63 \%(\mathrm{P}<0.01)$ in heart of the euthermic control value (Fig. 3.11B). 


\section{DISCUSSION}

A large amount of research has established that perturbations of ER function lead to the accumulation of unfolded proteins in the ER lumen, which triggers the UPR and results in a variety of responses that facilitate recovery. These include (a) reducing folding demand on the ER by suppressing the transcription/translation of genes to reduce the input of nascent proteins, (b) increasing the clearance of slowly folding or misfolded proteins by enhancing the ERAD pathway, and (c) increasing folding capacity by synthesis of chaperones and foldases (Luo et al., 2003; Schröder and Kaufman 2005; Lee, 2005). During hibernation, all physiological and metabolic activities are profoundly depressed in organs (Storey, 2003), resulting in conditions of near $0^{\circ} \mathrm{C}$ body temperatures and organ hypoperfusion during torpor as well as pronounced oxidative stress during arousal (when $\mathrm{O}_{2}$ consumption increases massively). All of these conditions can cause major metabolic damage for nonhibernating species and lead to an activation of the UPR. Thus, I hypothesized that ground squirrels would need to modify their UPR response in order to deal with a potential disruption of ER function that could arise under the physiological conditions that accompany hibernation. Is activation of the UPR an adaptive response that helps maintain ER stability during torpor? Elevated antioxidant defenses are now known to be an important adaptation for hibernation success; increased levels of antioxidant metabolites (e.g. ascorbate), elevated activities of antioxidant enzymes, and hibernation responsive up-regulation of various antioxidant genes have now been reported (Buzadzic et al., 1990; Drew et al., 2002; Storey, 2003; Eddy et al., $2005 b$ ). The present study looks at another protective response that allows cells to deal 
effectively with stress - the UPR - and evaluates its potential role in stabilizing intracellular proteins during hibernation.

Data presented in the previous chapter, demonstrated that one of the key components of the UPR, an up-regulation of GRP78, occurs during hibernation in BAT and brain of thirteen-lined ground squirrels (also see Mamady and Storey, 2006). Both grp78 mRNA and GRP78 protein were elevated during hibernation and this suggests that ER stress occurs during torpor. Since GRP78 production is a key biomarker for the onset of the UPR, I next evaluated other proteins that are known to be involved in the UPR and determined their responses during hibernation. These included pPERK, ATF6-50 kDa, ATF4 (and selected co-factors CREB-1 and p-CREB1), CHOP and GADD34.

Phosphorylation of the ribosomal initiation factor eIF $2 \alpha$ blocks the delivery of the initiating Met-tRNA to the ribosomal preinitiation complex and studies with multiple ground squirrel tissues, including brain, have shown that the amount of phospho-eIF $2 \alpha$ increases strongly during hibernation as one of the mechanisms of metabolic rate depression (Storey and Storey, 2004; Hittel and Storey, 2002; Frerichs et al., 1998). The phosphorylated form of PERK (pPERK) has been implicated in the inhibition of translation by phosphorylating eIF2 $\alpha$ at Ser-51 (Koumenis et al., 2002). However, western blot analysis of pPERK expression levels in ground squirrel brain found no difference between euthermic and hibernating states (Fig. 3.2A) which suggests that pPERK is not the kinase responsible for eIF2 $\alpha$ inactivation during torpor. Consistent with this, Jiang and Wek (2005) reported that deletion of PERK in MEF cells treated with a proteasome inhibitor that can trigger ER stress, did not diminish eIF2 $\alpha$ phosphorylation in that system. However, eIF $2 \alpha$ can be phosphorylated by a variety of other protein 
kinases such as GCN2, PKR and HRI (Clemens, 2001) so one of these may mediate this action during hibernation.

A second signaling molecule involved in the UPR is the transcription factor ATF6. It is constitutively expressed as a $90 \mathrm{kDa}$ protein, which is converted to a $50 \mathrm{kDa}$ active protein in ER-stressed cells and moves into the nucleus to induce transcription of downstream genes including grp78, grp94, CHOP and XBP-1 (Wang et al., 2000; Yoshida et al., 2000; Shen and Prywes, 2005). The present data show that overall protein levels of ATF6-50 kDa increased slightly by $20 \%$ in skeletal muscle during hibernation whereas no change in ATF6-50 kDa expression occurred in brain and heart (Fig. 3.2B). However, the critical determinant of ATF6-50 $\mathrm{kDa}$ function is whether the transcription factor moves into the nucleus during hibernation. Analysis of ATF6-50 kDa distribution in cytoplasmic versus nuclear fractions found an elevated nuclear content of ATF6-50 $\mathrm{kDa}$ (by 1.45 -fold) in heart during hibernation but ATF6 distribution was unaltered in brain between the euthermic and hibernating states and in skeletal muscle the observed increase in total ATF6-50 kDa appeared to be due to an increased cytoplasmic content of the transcription factor (Fig. 3.9B). The higher amount of ATF6-50 kDa that is translocated into the nuclear fraction of heart during hibernation suggests that ATF6-50 $\mathrm{kDa}$ mediated gene expression would be enhanced by binding to the ER stress element of selected genes. Heart has a critical function to play in hibernation because blood pumping has to be maintained throughout torpor; indeed, although heart rate is extremely low in torpor, peripheral resistance increases substantially and to compensate for this the force of contraction actually increases (Wang, 1989). It is not surprising, then, that myosin restructuring occurs in heart during hibernation, involving the hibernation-responsive up- 
regulation of selected myosin isoforms (Storey and Storey, 2004). In skeletal muscle, the elevated content of ATF6-50 $\mathrm{kDa}$ in the cytoplasm indicates that there is perhaps no change in ATF6-mediated gene expression during torpor but high cytoplasmic ATF6-50 might be put to use when the ground squirrel arouses from torpor in order to support a rapid increase in gene expression at that time.

The main pathway of the UPR that was investigated in this study is the PERK/eIF2 $\alpha /$ ATF4 pathway. Evidence has been presented that ATF4 requires eIF2 $\alpha$ phosphorylation for its translation upon various stress conditions including oxidative stress in nonhibernating mammals (Harding et al., 2000). Although pPERK levels did not change during hibernation in brain (Fig. 3.2) and kidney (data not shown), high levels of phosphorylated eIF $2 \alpha$ have been reported in both tissues of hibernating ground squirrels compared with euthermic animals (Frerichs et al. 1998; Hittel and Storey, 2002). Thus, an assessment of ATF4 expression levels in ground squirrel tissues was justified (Fig. 3.3B). The data show that ATF4 was markedly elevated by $1.9-2.5$ fold in BAT, brain and muscle during hibernation. These results suggest an important role for ATF4 in torpor and implicate the downstream genes of ATF4 as having potential roles in the hibernating phenotype. In order to determine whether the observed elevation of ATF4 protein levels resulted from increased transcription of the atf 4 gene, levels of atf4 mRNA transcripts were quantified using relative RT-PCR, in six tissues of euthermic versus hibernating ground squirrels. A $781 \mathrm{bp}$ cDNA from ground squirrel BAT was amplified and verified as encoding atf4. RT-PCR analysis of the amplified $781 \mathrm{bp}$ cDNA in ground squirrel tissues indicated no significant differences in atf4 message levels between the two states in BAT, brain, kidney or muscle but atf4 mRNA increased by 2.32 fold in liver 
and decreased in heart by $52 \%$ during torpor (Fig. 3.7). However, despite the high levels of atf4 message in liver, protein levels were unchanged in this organ between euthermic and hibernating conditions. Overall, then, the data suggest that the elevation of ATF4 protein in selected organs during hibernation does not occur at the transcriptional level but is a result of enhanced atf4 translation. The decreased level of atf 4 mRNA in heart correlates with the decreased level of ATF4 protein in the same organ.

A partial ground squirrel atf 4 sequence was retrieved that encoded $\sim 89 \%$ of protein (including the C-terminal) as compared with the full sequence from other mammals (Fig. 3.5). The squirrel ATF4 protein sequence showed high identity (82 to 86\%) with the human, rat and mouse sequences (Fig. 3.6). The partial ATF4 sequence covered the basic leucine zipper (bZIP) domain at the carboxyterminus that is important for the function of a transcription factor. Selected amino acid substitutions occurred in that region, including two changes of particular interest; the substitution of a hydrophobic isoleucine at position 311 for a more hydrophilic lysine residue and the substitution of a hydrophobic glycine at position 350 replacing a hydrophilic valine in the sequence of nonhibernating mammals. These changes might aid the binding of ATF4 to DNA and the transcriptional activity of ATF4 at low body temperatures; however, the purpose of these amino acid changes has not yet been confirmed.

Recent evidence indicates that CREB1, which is phosphorylated upon ER stress, forms a complex in vivo with ATF4 to induce transcription of a set of downstream genes including grp78 (Luo et al., 2003). Western blotting was used to measure total CREB1, as well as its active form, phosphorylated CREB1, in ground squirrel tissues. Total CREB1 content was largely unaffected during torpor but p-CREB1 increased 
significantly in all organs tested during hibernation, rising between 2 and 16 fold (Fig. 3.8). This indicates that $E R$ stress occurring during hibernation induces the phosphorylation of CREB 1 and mediates transcriptional activation of downstream genes via this route. These data are also consistent with studies on another hibernator that found 2-7 fold higher levels of phospho-CREB1 during hibernation in organs of Richardson's ground squirrels (S. richardsonii) (MacDonald and Storey, 2005).

Elevated levels of both ATF4 and p-CREB1 in selected organs of hibernators implicates their importance but both need to migrate to the nucleus to influence gene expression during hibernation. Hence, subcellular fractionation was used to analyze the distribution of these transcription factors between cytoplasmic and nuclear fractions of brain and skeletal muscle in euthermic and hibernating squirrels. These studies showed moderate, but significant, elevation of nuclear ATF4 content and extremely large increases in nuclear p-CREB1 abundance during hibernation (Fig. 3.10). As a coactivator of ATF4, the translocation of p-CREB1 into nuclei during hibernation would strongly increase the amount of ATF4•p-CREB1 complex present and thereby increase ATF/CRE binding and the activation of genes under the control of these transcription factors. Hence, elements of the UPR that provide protection to ER proteins during hibernation (such as enhanced GRP78 expression) appear to be under the control of ATF4 and pCREB1 transcription factors.

Furthermore, Western blot analysis of others genes involved in the UPR, such as CHOP, revealed a higher expression level in heart during hibernation. Oppositely, GADD34 which plays a role in recruiting protein phosphatase 1 (PP1) to dephosphorylate eIF $2 \alpha$, showed a reduced level in brain and heart of hibernating squirrels compared to 
euthermic animals. Future studies remain to be done to determine whether an elevated level of CHOP leads to some level of apoptosis in heart during hibernation and how the cells balance between apoptosis and cell survival. Moreover, it will be interesting to determine whether the IRE1 pathway of the UPR is activated to enhance protein degradation in heart of hibernating squirrels. During deep torpor, protein translation is inhibited via reversible phosphorylation control of eIF2 $\alpha$ and GADD34 expression levels were lower or unchanged in the tissues studied. However, during brief periods of arousal that last between 12-24 hours, protein synthesis resumes and under those conditions, GADD34 might be activated. Thus, GADD34 could represent the intermediate signal for the switch from global translation inhibition to the resumption of general translation as animals arise from torpor. This is consistent with the results of $\mathrm{Ma}$ and Hendershot (2003) and Blais et al. (2004) who have reported that GADD34 forms a negative feedback regulatory loop and that its upregulation mediates eIF $2 \alpha$ dephosphorylation followed by inhibition of ATF4 expression and, thereby, mediates cellular recovery after ER stress.

In summary, the results presented in this chapter demonstrate that expression of ATF4 increased in BAT, brain and skeletal muscle during hibernation, despite the unchanged expression of pPERK, one of the eIF2 $\alpha$ kinases. The data suggest that the increase in ATF4 protein levels is due to enhanced translation of atf4 message rather than increased transcription of the atf4 gene. Protein synthesis is strongly suppressed in torpor and one mechanism that would contribute to this is the reduced levels of GADD34 protein that would help to keep eIF $2 \alpha$ in the phosphorylated inactive state during torpor. It will be interesting to more deeply investigate the ATF6 pathway of the UPR in heart of 
hibernating squirrels since ATF6-50 kDa showed a translocation from the cytoplasm into the nucleus of hibernating heart. ATF4 involvement in ER stress links both branches of the UPR with the induction of the pro-apoptotic CHOP protein and with pro-survival proteins such as GRP78 (Mamady and Storey, 2006). ATF4 upregulation, accompanied by its nuclear cofactor p-CREB1, may mediate adaptive responses to the adverse conditions of stresses occurring during hibernation in the ground squirrels. Whether the PERK/eIF2 $\alpha /$ ATF4 signaling pathway is a key element that promotes cell survival during hibernation and how the stressed cells balance between the survival and the death responses still needs to be determined. By understanding the patterns of gene expression in the UPR pathway in mammalian hibernation, we can gain knowledge of both the key metabolic problems faced by different organs and the natural solutions that have been developed. Interestingly, a recent study on ER stress and oxidative stress has linked ATF4 and Nrf2 signaling pathways, suggesting that phosphorylated form of Nrf2 might also participate in ER stress signaling by promoting expression of genes encoding antioxidants, genes implicated in cell growth, protein folding and cell survival (Cullinan and Diehl, 2006). 
Fig. 3.1: Model of UPR signaling in mammalian cells, depicting role of ATF4 during ER stress. Upon ER stress, the signaling molecules: PERK, IRE1 and ATF6 dissociate from GRP78 and undergo posttranslational modifications that activate them. Phosphorylated PERK (pPERK) triggers inhibition of ribosomal protein translation via phosphorylation of the $\alpha$-subunit of the eukaryotic initiation factor-2 $\alpha$ (eIF2 $\alpha$ ). Phosphorylated eIF2 $\alpha$ then enhances expression of the activating transcription factor 4 (ATF4) which activates both pro-survival and pro-apoptotic genes that participate in the UPR. Adapted from Blais et al. (2004). 
Fig. 3.1:

\section{MODEL OF UNFOLDED PROTEIN RESPONSE}

ATP depletion - ER Calcium depletion - Increase in ER lumenal reducing conditions Amino acid deprivation - Hypoxia - Ischemia - Oxidative stress

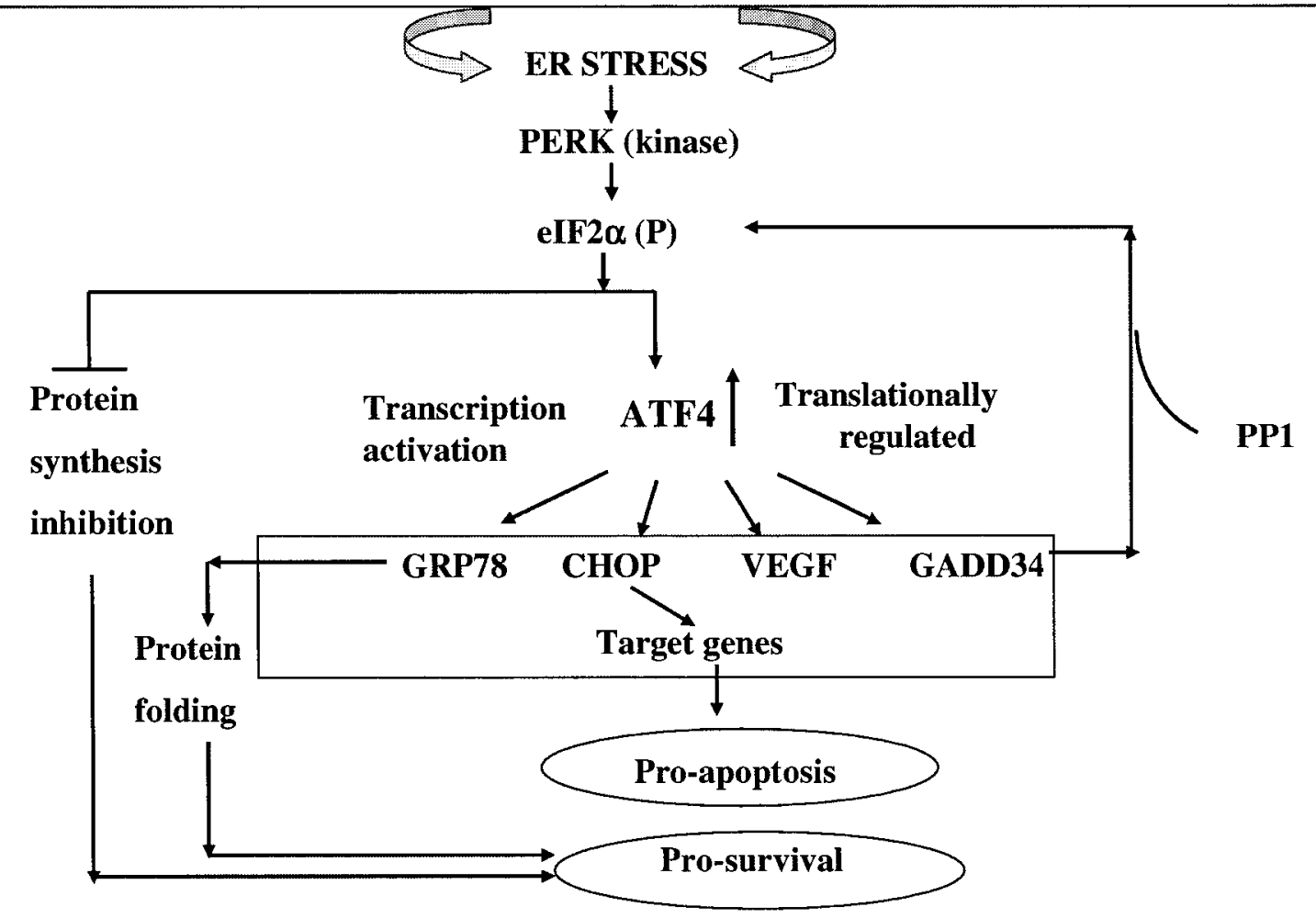


Fig. 3.2: (A) Immunoblot analysis of p-PERK levels in brain of euthermic and hibernating ground squirrels. Equal amounts of protein were loaded into each lane of $8 \%$ SDS-polyacrylamide gels, followed by transfer to PVDF membranes, probing with antip-PERK antibodies, and quantification by densitometry. (B) Comparable Western blot analysis of ATF6 levels in three tissues from euthermic $(\mathrm{E})$ and hibernating $(\mathrm{H})$ ground squirrels; separation was on $12 \%$ SDS-PAGE gels. Histograms show mean ratios \pm S.E.M., $n=3$ independent trials on tissue from different animals. * - Hibernating sample is significantly different from the corresponding euthermic value using the two-tailed Student's $t$-test, $\mathrm{P}<0.05$. 
Fig. 3.2:

A.
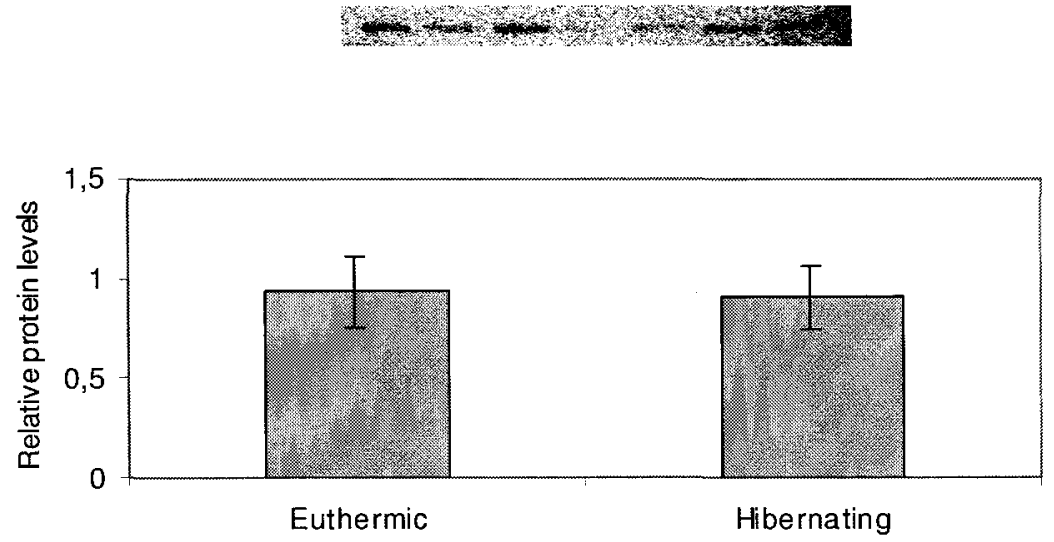

B.

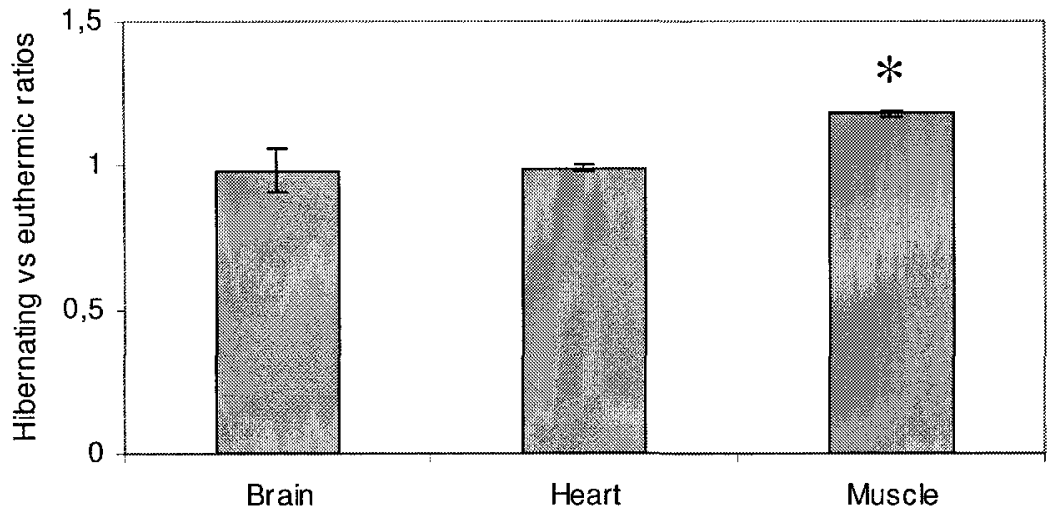


Fig. 3.3: (A) Representative Western blots showing ATF4 protein levels in six tissues from euthermic $(\mathrm{E})$ and hibernating $(\mathrm{H})$ ground squirrels. (B) Histograms show the ratio hibernating:euthermic ATF4 levels. B.A.T. is brown adipose tissue. Data are means \pm S.E.M, $\mathrm{n}=3$ independent trials on different animals. *, $* *$ - Values for hibernating samples are significantly different from the corresponding euthermic values using the two-tailed Student's $t$-test, $\mathrm{P}<0.05$ and $\mathrm{P}<0.01$, respectively. 
Fig. 3.3:

A.

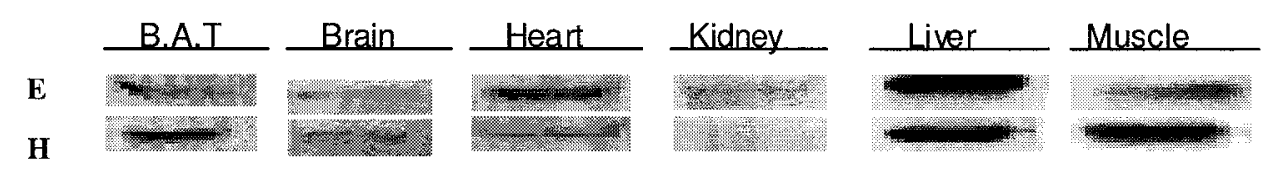

B.

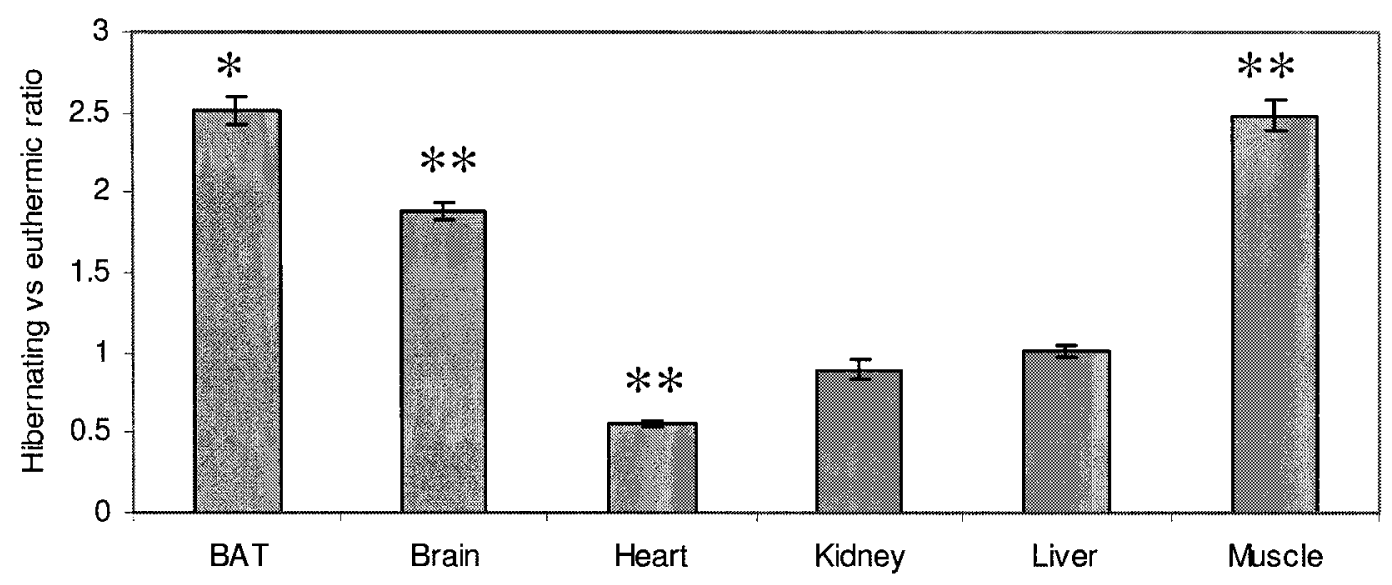


Fig. 3.4: Partial cDNA sequence of atf4 amplified from brown adipose tissue of thirteenlined ground squirrels along with the translated amino acid sequence. Nucleotides and amino acids are numbered on the left. A single open reading frame was predicted from the nucleotide sequence and encoded a polypeptide with 313 residues. The stop codon is underlined in the cDNA sequence. 
Fig. 3.4 :

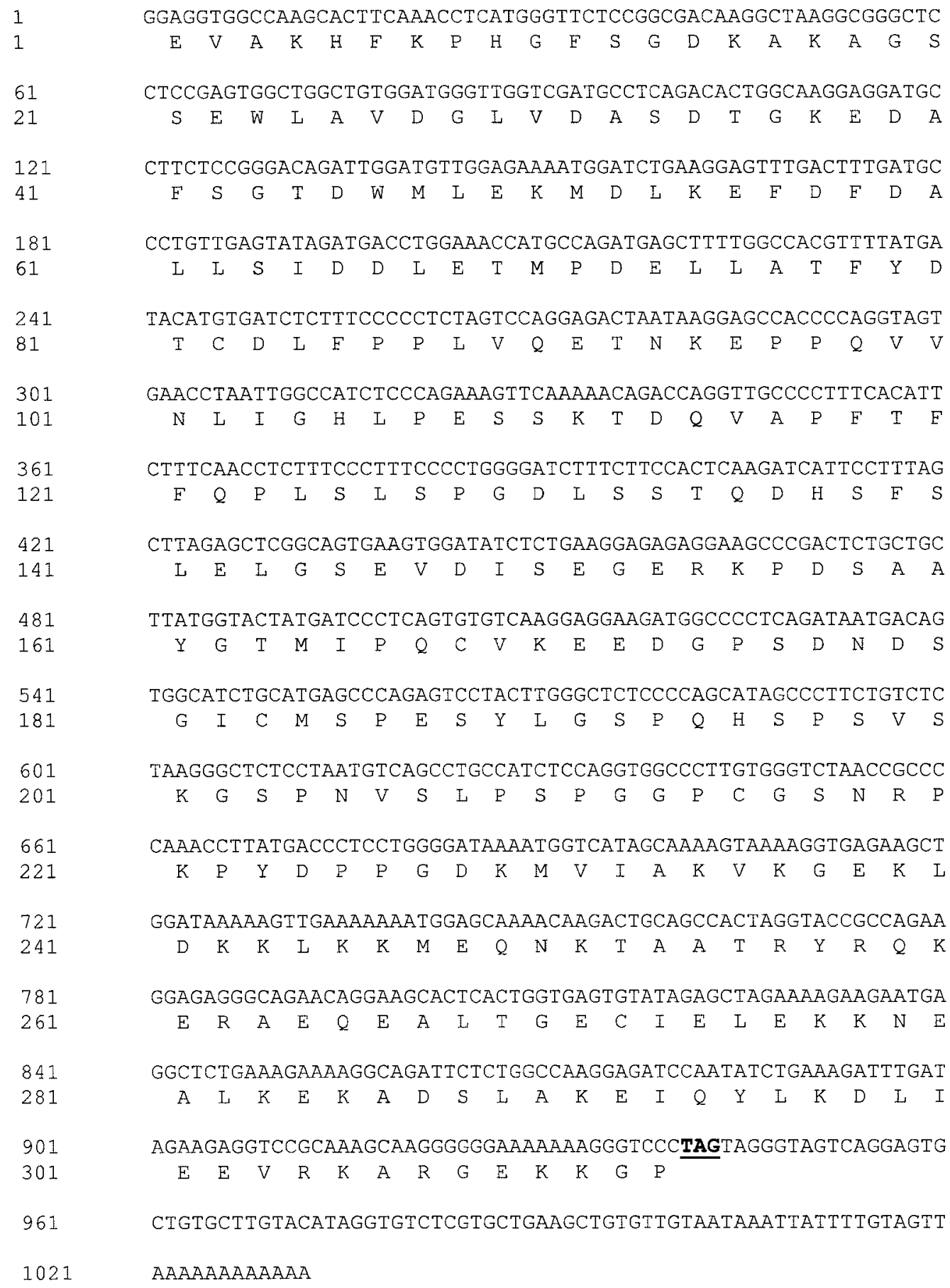


Fig. 3.5: Ground squirrel (Spermophilus tridecemlineatus) ATF4 partial amino acid sequence (GenBank accession number DQ324000) aligned with human (Homo sapiens), mouse (Mus musculus), and rat (Rattus norvegicus) sequences (Genbank accession numbers NM_182810, BC085169 and NM_024403, respectively). Unique amino acid substitutions in the squirrel sequence are indicated in bold underline. Dashes (-) represent amino acids in the human, mouse or rat sequences that are identical with the squirrel sequence. Periods are present in the alignment to indicate where an amino acid is not present in the coding region of one of the species. 
Fig. 3.5:

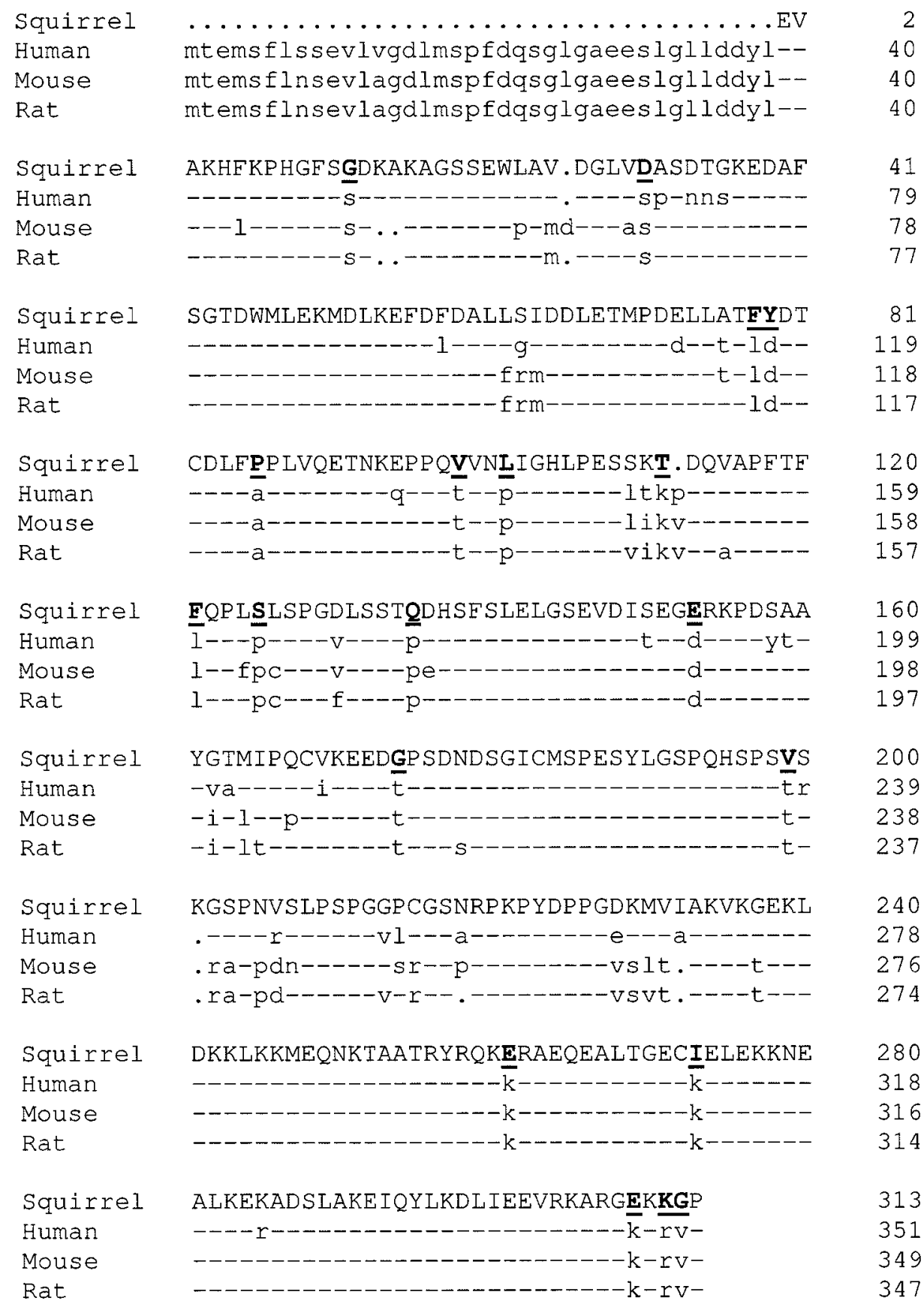


Fig. 3.6: (A) Cladogram prepared with DNAMAN depicting amino acid identity of the ground squirrel ATF4 partial sequence (Spermophilus tridecemlineatus, accession number: DQ324000) compared with ATF4 protein of other mammalian species: human (Homo sapiens, accession number: NM_182810), mouse (Mus musculus, accession number: BC085169) and rat (Rattus norvegicus, accession number: NM_024403). (B) Homology matrix of the four sequences. 
Fig. 3.6:

A.
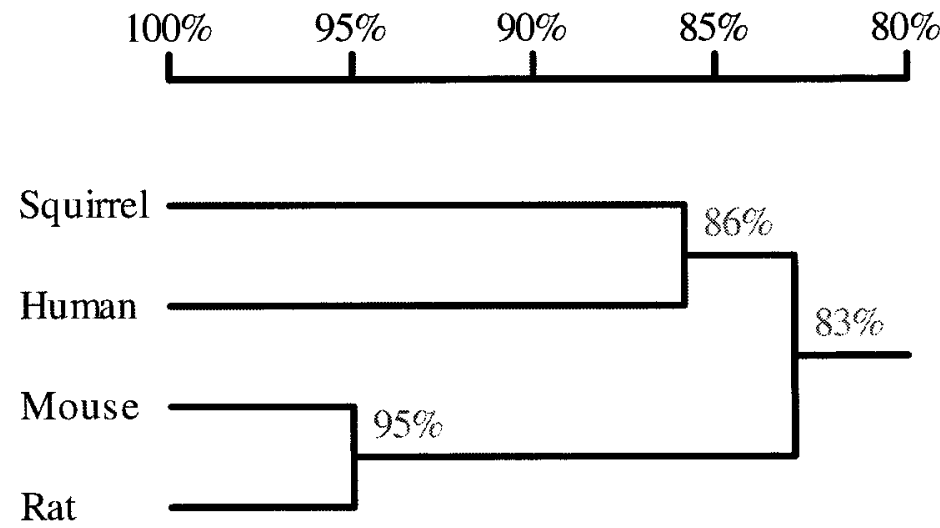

B.

$\begin{array}{lll}\text { Homology matrix of } 4 & \text { sequences } \\ & \\ \text { Squirrel } & 100 \% & \\ \text { Human } & 85.6 \% 100 \% & \\ \text { Mouse } & 81.9 \% 84.5 \% 100 \% \\ \text { Rat } & 83.1 \% 84.4 \% 94.8 \% 100 \%\end{array}$


Fig. 3.7: (A) Effect of hibernation on atf4 mRNA levels in six tissues of thirteen-lined ground squirrels. (B) Histogram showing the ratio of normalized PCR product levels (relative to $\alpha$-tubulin) in tissues from hibernating versus euthermic ground squirrels. The highest dilutions of atf $4\left(10^{-4}\right.$ or $\left.10^{-5}\right)$ and $\alpha$-tubulin (from the same sample tube) which gave visible PCR product bands were chosen for quantification. Data are mean $\pm S E M, n$ $=3$ independent trials on different animals. $* *$ - Hibernating values are significantly different from the corresponding euthermic values, $\mathrm{P}<0.01$. 
Fig. 3.7:

A.

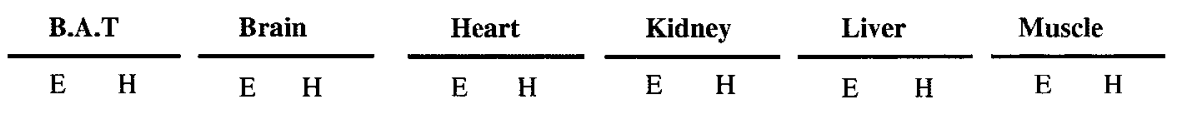

ATFA

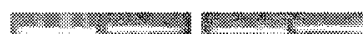

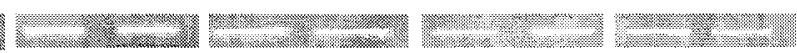

$\alpha$-Tubulin

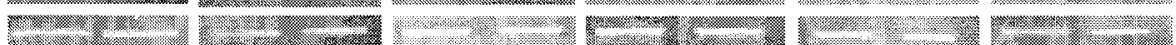

B.

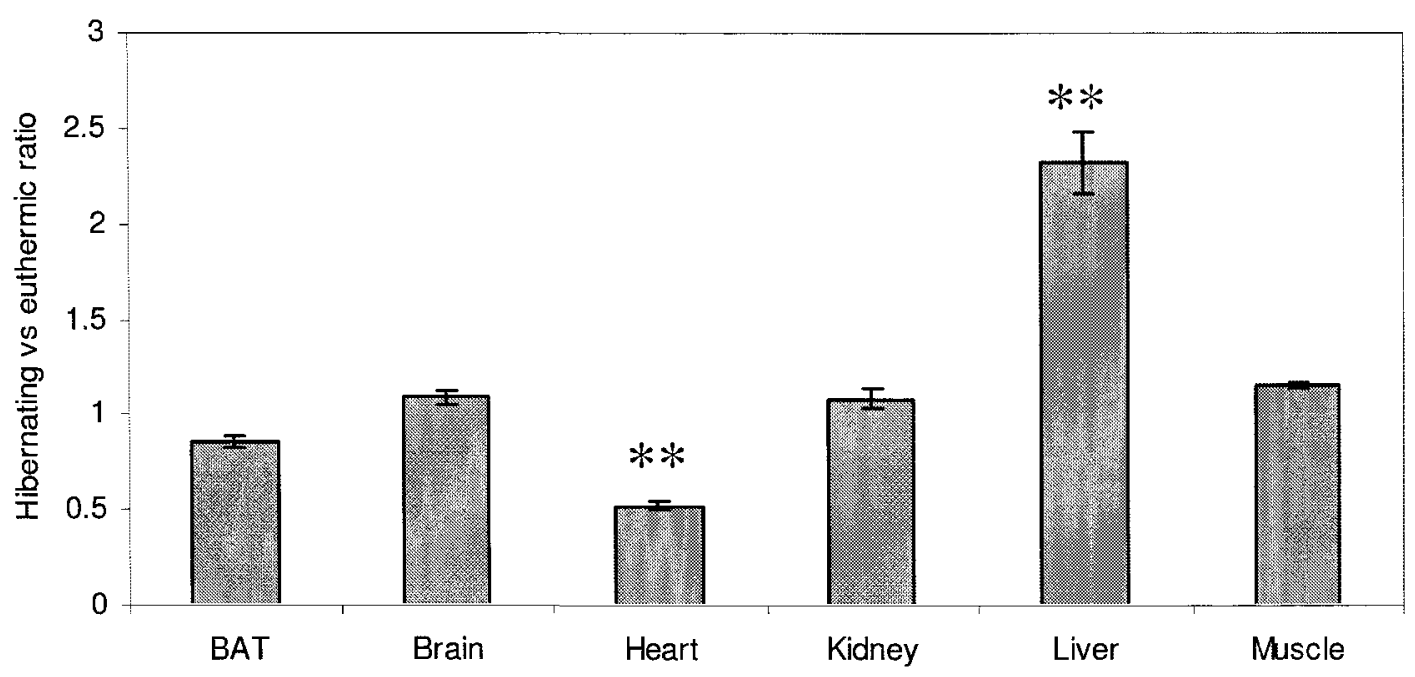


Fig. 3.8: (A) Western blot analysis of total CREB-1 protein and phosphorylated CREB-1 levels in tissues from euthermic and hibernating ground squirrels. (B) Histograms show the ratio hibernating:euthermic for $\mathrm{p}$-CREB-1 levels. Data are means \pm S.E.M., $n=4$ independent trials on different animals. *,** - Values for hibernating samples are significantly different from the corresponding euthermic values using the two-tailed Student's $t$-test, $\mathrm{P}<0.05$ and $\mathrm{P}<0.01$, respectively. 
Fig. 3.8:

A.
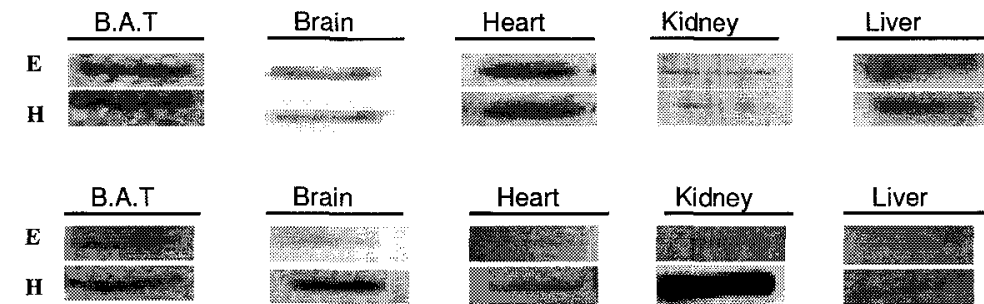

Muscle
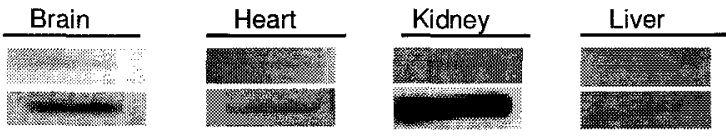

Muscle

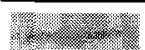

(3). 2.1012

$\mathrm{p}-\mathrm{CREB} 1$

B.

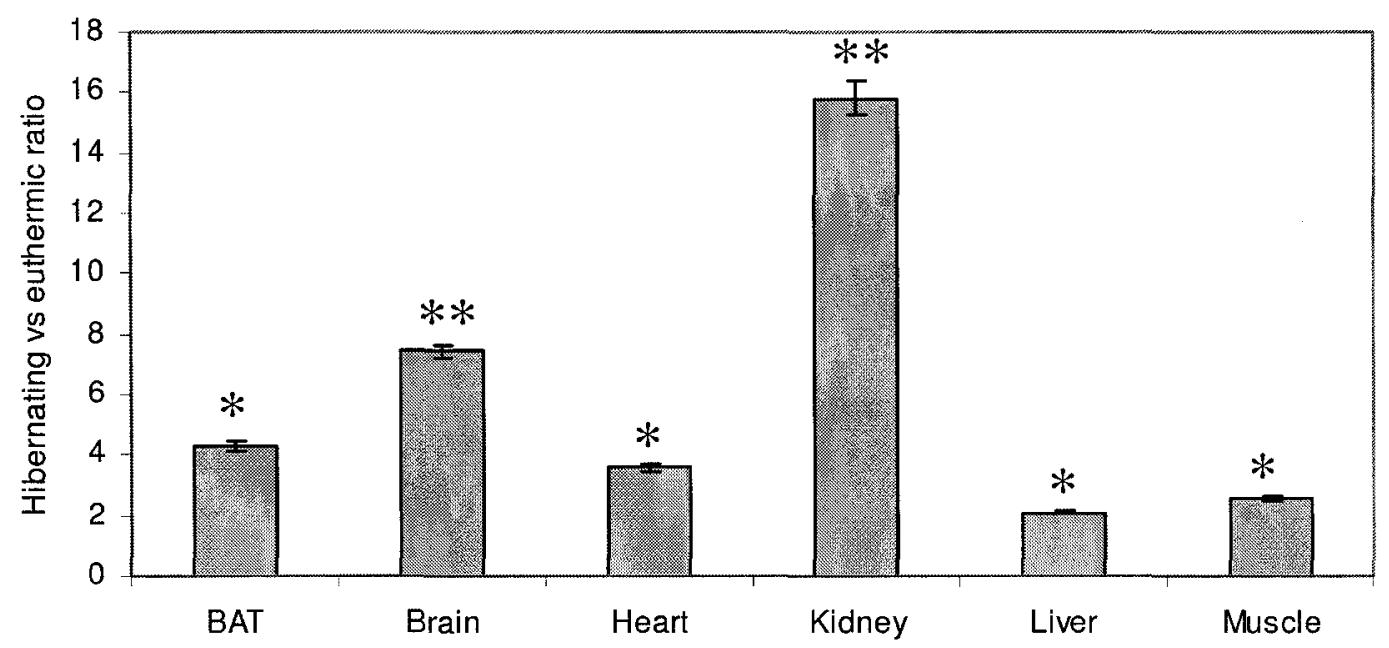


Fig. 3.9: Analysis of ATF6 $(50 \mathrm{kDa})$ protein distribution between cytoplasmic and nuclear fractions in three tissues of euthermic $(\mathrm{E})$ and hibernating $(\mathrm{H})$ ground squirrels. (A) Representative Western blots showing detection of ATF6 in nuclear and cytoplasmic extracts. (B) Histograms showing relative levels of ATF6 in cytoplasmic and nuclear fractions from each tissue. Data are means \pm S.E.M., $n=3$ independent trials on different animals. $* * *$ - Values for hibernating samples are significantly different from the corresponding euthermic values using the two-tailed Student's $t$-test, $\mathrm{P}<0.05$ and $\mathrm{P}<$ 0.01 , respectively. 
Fig. 3.9:

A.

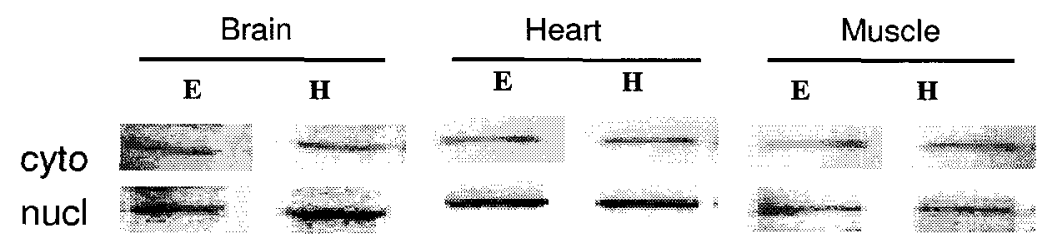

B.

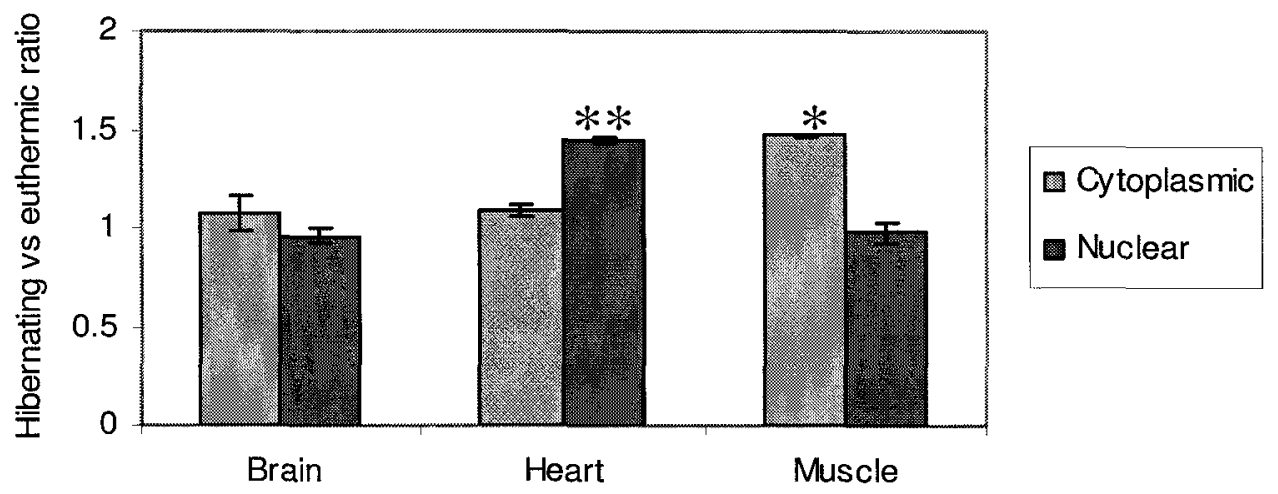


Fig. 3.10: Distribution of the transcription factors ATF4 (A) and p-CREB1 (B) between cytoplasmic and nuclear fractions of brain and muscle from euthermic and hibernating ground squirrels. Histograms show the relative levels of each protein in ground squirrel organs. Data are means \pm S.E.M., $\mathrm{n}=3$ independent trials on different animals. ${ }^{*},{ }^{*}$ Values for hibernating samples are significantly different from the corresponding euthermic values using the two-tailed Student's $t$-test, $\mathrm{P}<0.05$ and $\mathrm{P}<0.01$, respectively. 
Fig. 3.10:

A.

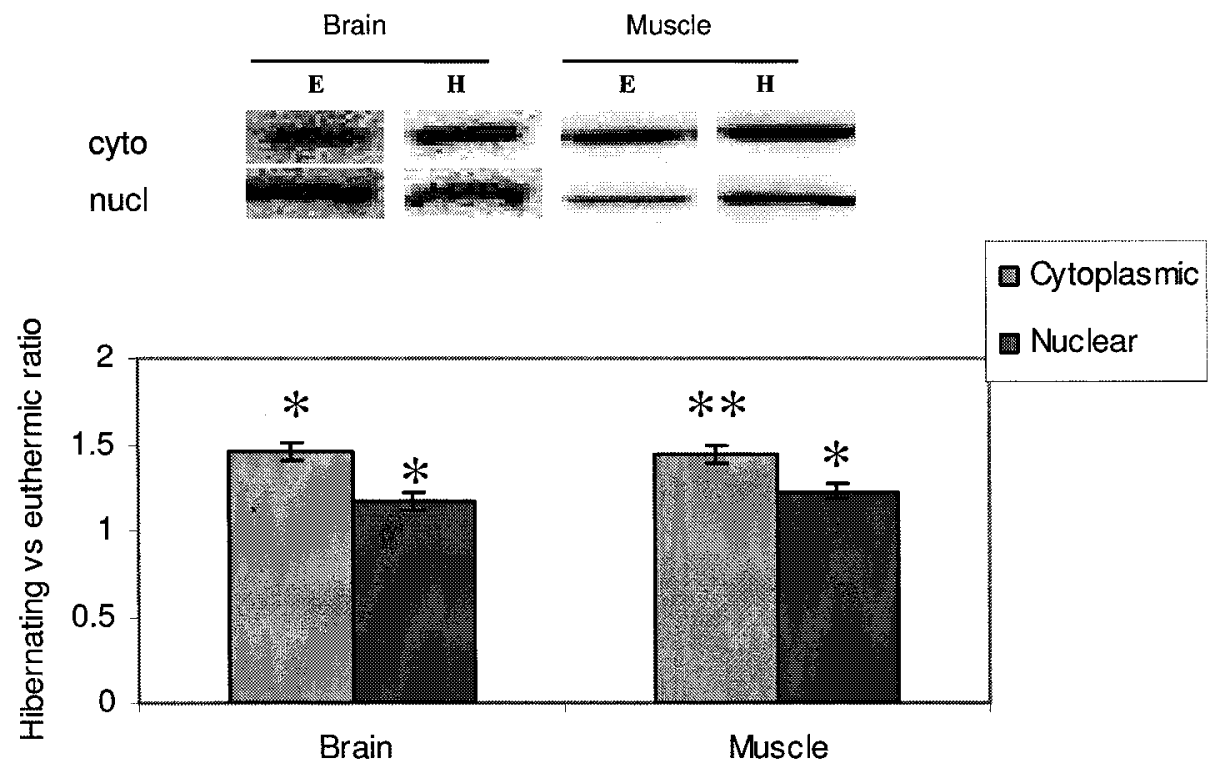

B.
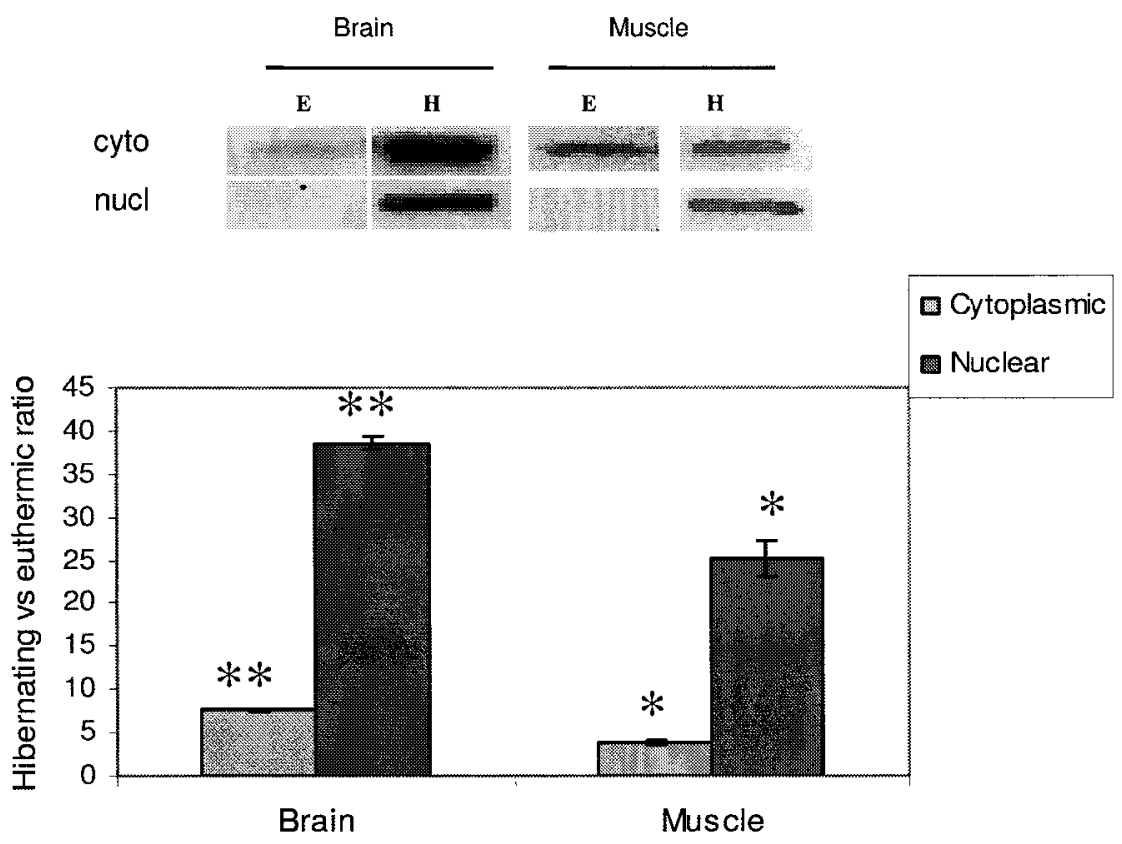
Fig. 3.11: Representative Western blots of GADD153 (CHOP) (A) and GADD34 (B) protein levels in ground squirrel tissues. Aliquots $(15 \mu \mathrm{g})$ of total protein were electrophoresed on $10 \%$ SDS-polyacrylamide gels and incubated with anti-GADD153 and anti-GADD34. Histograms show the ratio of GADD153 and GADD34 protein levels in hibernating versus euthermic animals; data are means $\pm S E M, n=3$ independent trials on different animals. $* * *$ - Values for hibernating sample are significantly different from the corresponding euthermic values, $\mathrm{P}<0.05$ and $\mathrm{P}<0.01$, respectively. 
Fig. 3.11:

A.
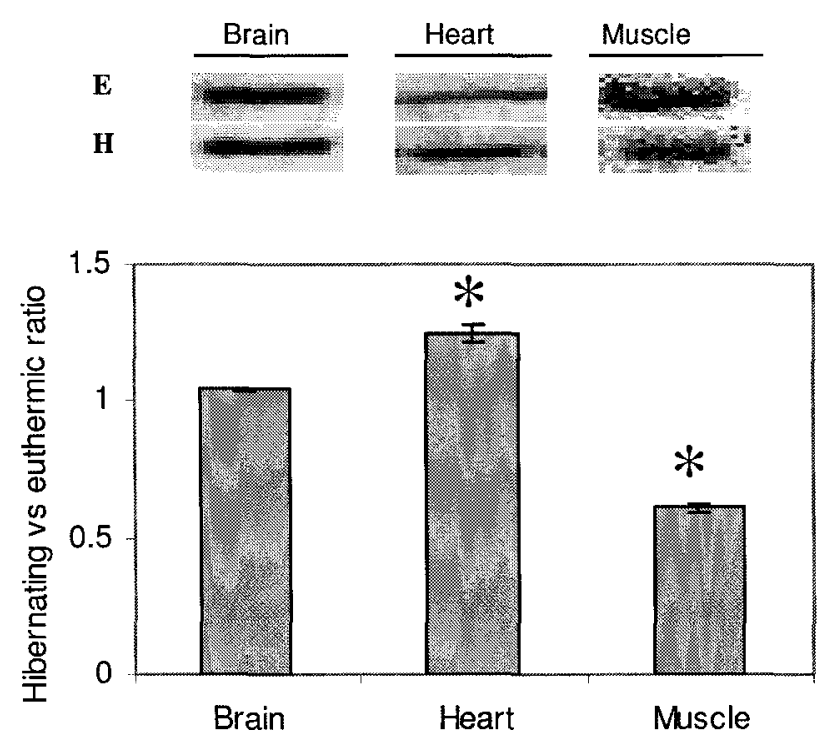

B.
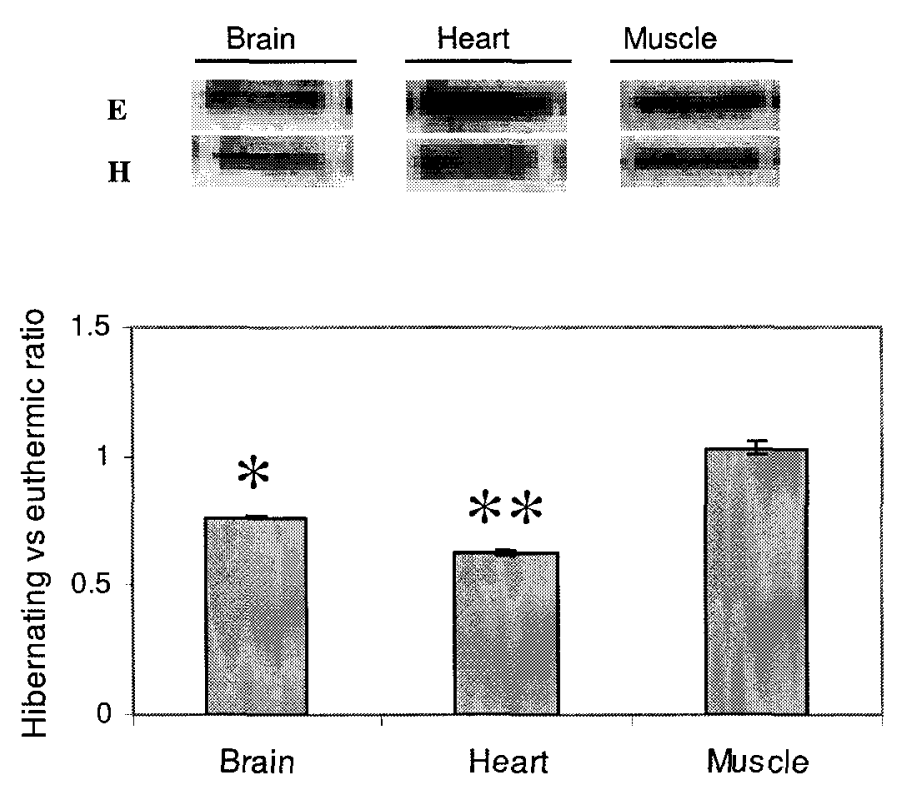


\title{
Chapter 4
}

\section{Muscle disuse atrophy:}

profile and assessment of FoxO1a activation,

\author{
MAFbx/atrogin-1, Bcl-3 and NFKB p50 in skeletal
}

\author{
muscle and heart during hibernation
}




\section{INTRODUCTION}

Muscle atrophy is defined as the changes that occur in muscles of adult animals as a consequence of disuse brought about by factors such as muscle unloading, immobilization and denervation, and also conditions of aging, starvation and number of disease states (Jackman and Kandarian, 2004). Muscle atrophy is characterized by a decrease in protein content and fiber diameter; and develops when rates of protein degradation outstrip rates of protein synthesis (Cao et al., 2004; Thomason and Booth, 1990). Moreover, it has been reported for a number of animal models such as mice and rats that under conditions of mechanical unloading resulting from hindlimb suspension, the main constituent of muscle fibers (the myosin heavy-chain protein) shifts from slow to fast phenotype (Booth and Thomason, 1991). However, the hindlimb muscles of hibernating golden-mantled ground squirrel (Spermophilus lateralis) showed only slight atrophy during torpor, a much reduced level compared to the well-studied mammalian models (Rourke et al., 2004). Little is known about the mechanisms and regulatory pathway by which hibernators avoid severe disuse atrophy over the weeks of winter torpor during the hibernation period.

The molecular regulation of muscle atrophy implies different signaling pathways and molecular triggers. The IGF-1/PI3K/AKT (Insulin Growth Factor 1 / Phosphatidylinositol 3-Kinase / Protein kinase B) pathway, which has been shown to induce hypertrophy, is also involved in the inhibition of atrophy through AKT-mediated inhibition of the FOXO family of transcription factors (Stitt et al., 2004; Sandri et al., 2004; Nader, 2005). Forkhead box, class O (FOXO) proteins form a subfamily of the Forkhead family of transcription factors which are involved in tumorigenesis in 
rhabdomyosarcoma and acute leukemia (Anderson et al., 1998). Within this subfamily, three members: FKHR (Forkhead in Rhabdomyosarcoma, also called FoxO1a), AFX (acute-lymphocytic-leukaemia-1 fused gene from chromosome $\mathrm{X}$, also called FoxO4) and FKHRL1 (Forkhead in Rhabdomyosarcoma like 1, called FoxO3a), have sequence similarity to the nematode (Caenorhabditis elegans) homologue DAF-16 which was shown to mediate signaling via IGF1 (Insulin like Growth Factor 1), PI3K and AKT (Rena et al., 1999). To activate target genes, Forkhead transcription factors have been shown to bind to the insulin response elements (IREs) such as the one found in the promoter of the insulin-like growth factor-binding protein-1 (IGFBP-1). FOXO transcription factors also bind to a consensus core sequence of $5^{\prime}$-TTGTTTAC-3', which includes a sequence TRTTTAY (with $\mathrm{R}$ a purine base and $\mathrm{Y}$ a pyrimidine base) conserved among various Forkhead members (Furuyama et al., 2000). Moreover, beside its direct transcriptional activity, FoxO1a for example, interacts and co-operates with other proteins such as the proliferative-activated receptor- $\gamma$ co-activator 1 (PGC-1 $\alpha)$, in the activation of gluconeogenic genes, independent of DNA binding (Van der Heide et al., 2004). Furthermore, three putative AKT phosphorylation sites were found in human FoxO1a: Thr24, Ser256 and Ser319 (Barthel et al., 2005); and it has been reported that phosphorylation at these sites by AKT promotes cell survival and regulates the cell cycle (Brunet et al., 1999). In addition, phosphorylation at Ser256 results in FoxOla inactivation and release from DNA with a subsequent binding to $14-3-3$ proteins and export to the cytoplasmic compartment (Nader, 2005). However, in the absence of survival signals, cytoplasmic FOXO is dephosphorylated, causing dissociation from 143-3 proteins and allowing nuclear import of FOXO to activate gene expression. 
FOXO transcription factors are involved in regulation of the cell cycle, cell death, cell metabolism and oxidative stress (Burgering and Kops, 2002). In recent years, evidence has accumulated that, through the PI3K/AKT pathway, FoxO1a is also involved in the regulation of skeletal muscle mass (Kamei et al., 2004) and in the development of muscle atrophy by inducing atrophy-related genes, including the atrophy-related ubiquitin ligase atrogin-1 or Muscle Atrophy F box (MAFbx) (Stitt et al., 2004; Sandri et al., 2004). MAFbx is a protein that binds and mediates ubiquitination of specific substrates, through protein-protein interaction domains, to target them for degradation (Jackson et al., 2000).

Another pathway that may be involved in muscle atrophy is the NFKB pathway. Two members of the NFKB/IKB family of transcriptional regulators, NFKB1 (p50) and Bcl-3 (B cell lymphoma 3), have been implicated. Recent work has demonstrated that both genes were required in muscle disuse atrophy and that p50 and $\mathrm{Bcl}-3$ form a complex that translocates into the nucleus to transactivate target genes necessary for the induction of muscle atrophy (Hunter and Kandarian, 2004).

Given the evidence that hibernating mammals experience a dramatic reduction in locomotor activity (Yacoe, 1983; Rourke et al., 2004) and that the cardiac muscle in several hibernating rodents maintains mass (Wickler $e t$ al., 1991) whereas skeletal muscle shows slight atrophy, I hypothesized that selected genes involved in muscle disuse atrophy may be down-regulated and their protein products inactivated during torpor to minimize atrophy and defend muscle functions.

The current experiments were designed to investigate the regulation of gene and protein expression of FoxO1a in heart and skeletal muscle of hibernating ground 
squirrels, Spermophilus tridecemlineatus, as well as protein levels of its downstream gene MAFbx/atrogin-1. Furthermore, protein expression and translocation of NFkB p50 and Bcl-3, members of the NFKB regulatory pathway, were also determined to assess their involvement in the regulation of muscle mass during hibernation.

\section{MATERIALS AND METHODS}

\section{Animals}

All animals were captured, housed and organs harvested as previously described in Chapter 2. The skeletal muscle used is a mixture of several hindlimb muscles.

\section{RNA isolation}

Total RNA was isolated from heart and skeletal muscle of euthermic and hibernating ground squirrels using Trizol reagent (Invitrogen) (100 $\mathrm{mg}$ tissue per $\mathrm{ml}$ ) according to the manufacturer's protocol as previously described in Chapter 2 .

\section{cDNA synthesis and PCR amplification of FoxOIa}

First strand synthesis was performed as described previously (Chapter 2). Serial dilutions of cDNA from $10^{-1}$ to $10^{-4}$ were then prepared before performing relative RTPCR. Specific primers were designed for the FoxOla sequence from 13-lined ground squirrels (accession number: AY255525), as reported by Cai et al. (2004). Primers were synthesized by Sigma Genosys. The sequences were: 5'-TCCAGCATGAGCCCTTTG-3' (forward) and 5'-CAATGTGTGGGAaGCTTTGG-3' (reverse). Primers for the housekeeping gene, $\alpha$-tubulin, which was used as an internal control for quantification 
were: forward (5' - AAGGAAGATGCTGCCAATAA - 3') and reverse (5' GGTCACATTTCACCATCTG- 3'). The cycling conditions for semi-quantitative PCR were as follows: $95^{\circ} \mathrm{C}$ for $4 \mathrm{~min}$, then 35 cycles of $95^{\circ} \mathrm{C}$ for $30 \mathrm{sec}, 59^{\circ} \mathrm{C}$ for $30 \mathrm{sec}$ and $72^{\circ} \mathrm{C}$ for $30 \mathrm{sec}$, followed by a final step at $72{ }^{\circ} \mathrm{C}$ for $7 \mathrm{~min}$. The PCR products were separated in a $1 \%$ agarose/ethidium bromide gel. A single product with a size of $729 \mathrm{bp}$ was obtained with FoxOla primers and 635 bp product with $\alpha$-tubulin primers. Band densities were scanned using the UV transilluminator program of the ChemiGenius imaging system and quantified with the associated Gene Tools software (Syngene, MD, USA). From a comparison of the band intensities of PCR products at each dilution, the lowest dilutions for alpha-tubulin and FoxOla that gave visible bands were chosen for quantification and the relative amounts of FoxOla mRNA transcripts, relative to alphatubulin transcripts, were determined. The relative mRNA levels for hibernating versus euthermic states were calculated as previously described in Chapter 2:

$$
\text { Relative mRNA content }=\frac{\text { (hibernating FoxOla /hibernating } \alpha \text {-tubulin) }}{\text { (euthermic FoxOla /euthermic } \alpha \text {-tubulin) }}
$$

Statistical analysis of normalized band intensities from control versus hibernating samples used the Student's $t$-test. The mean ratios of hibernating to euthermic values and the S.E.M on the ratios were then calculated, and the data are shown as mean ratios \pm S.E.M in histograms.

\section{Western Blotting}

Western blotting was performed as described in Chapter 2. Total protein was 
isolated from samples of heart and skeletal muscle of euthermic and hibernating ground squirrels. Aliquots containing $75 \mu \mathrm{g}$ of protein were loaded into each well of $10 \%$ SDS gels, followed by electrophoresis, and then transfer to PVDF membrane. Rabbit polyclonal antibodies to FKHR (FoxO1a) and phospho-FKHR (Ser256) (pFoxO1a) were purchased from Cell Signaling Technologies. Anti-goat MAFbx antibody, rabbit polyclonal antibody against Bcl-3 and anti-goat NFkB p50 antibody were all purchased from Santa Cruz Biotechnologies. Prior to the addition of primary antibody, blots were blocked for 15-30 min in TBST containing 2.5\% non-fat dried milk. All primary antibody incubations for Western blotting were carried out at $4^{\circ} \mathrm{C}$ overnight in 1X TBST at 1:1000 v/v dilution for FoxO1a and pFoxOla, or at 1:250 v/v dilution for MAFbx, Bcl-3 and $\mathrm{NF \kappa B}$ p50. Each blot was washed for $5 \mathrm{~min}$ in $1 \mathrm{X}$ TBST 3 times prior to the addition of secondary antibody. Secondary antibody (1:2000 v/v dilution) incubations were carried out for $90 \mathrm{~min}$ at room temperature and followed by 3 washes in $1 \mathrm{X}$ TBST for $5 \mathrm{~min}$ each. For FoxO1a, pFoxOla and Bcl-3 studies, HRP-linked anti-rabbit IgG (H\&L) secondary antibody (Cell Signaling) was used. For MAFbx and NFkB p50 studies, HRPlinked anti-goat IgG (H\&L) secondary antibody (Santa Cruz Biotechnologies) was used. Detection of signal on the PVDF membrane used an enhanced chemiluminescence (ECL) system as recommended by the manufacturer (Pierce). The membrane was scanned using the ChemiGenius (Syngene, MD, USA) and band densities on the resulting image were analyzed with the associated Gene Tools software.

\section{Distribution of FoxO1a and pFoxO1a in cytoplasmic and nuclear extracts}

Cytoplasmic and nuclear extracts from skeletal muscle were prepared as described 
in Chapter 2. Protein samples from each fraction $(75 \mu \mathrm{g})$ were separated on $10 \%$ SDSPAGE minigels at $180 \mathrm{~V}$ for $45 \mathrm{~min}$, transferred onto PVDF membranes, and immunoblotted with FoxO1a and pFoxO1a antibodies as described above.

\section{FoxOla DNA binding assay}

The TransAM FKHR, a commercially available kit for FKHR (FoxO1a) DNA binding assay was purchased from ActiveMotif. This kit contains a 96-well microplate to which the FoxOla consensus-binding site oligonucleotide was immobilized. Nuclear extracts were prepared from heart and skeletal muscle as described in Chapter 2 and then binding assays were performed according to the manufacturer's protocol. Briefly, $40 \mu \mathrm{l}$ of binding buffer containing 10 pmol of the mutated consensus oligonucleotide was added to each well. The mutated consensus oligonucleotide was provided with the kit as a nonspecific competitor for FoxO1 a that should have no effect on FoxO1a binding. A $40 \mu \mathrm{g}$ aliquot of nuclear extract in a total volume of $10 \mu 1$ lysis buffer was then added. FoxO1a binding was allowed to proceed for $1 \mathrm{~h}$ at $21^{\circ} \mathrm{C}$ with gentle shaking at $100 \mathrm{rpm}$. The microplates were then washed three times with $200 \mu \mathrm{l}$ washing buffer ( $10 \mathrm{mM}$ phosphate buffer, $\mathrm{pH} 7.5,50 \mathrm{mM} \mathrm{NaCl}, 0.1 \%$ Tween-20) added per well to remove unbound transcription factors. Anti-FoxO1a specific primary antibody diluted 1:500 v:v in antibody binding buffer was then added to each well and incubated for $1 \mathrm{~h}$ at $21^{\circ} \mathrm{C}$ without shaking. The wells were again washed three times with washing buffer and then secondary antibody (HRP-linked anti-rabbit IgG) was added to the wells in a 1:1000 v:v dilution in antibody binding buffer and incubated for $1 \mathrm{~h}$ at $21^{\circ} \mathrm{C}$ without shaking. The wells were then washed four times with washing buffer and then the manufacturer's 
developing solution $(100 \mu \mathrm{l})$ was added and incubated for $15 \mathrm{~min}$ in the dark. The stop solution $(100 \mu \mathrm{l})$ was then added, allowed to develop for $5 \mathrm{~min}$, and then color development was quantified spectrophotometrically by absorbance readings at $460 \mathrm{~nm}$.

\section{RESULTS}

\section{FoxO1a gene expression in ground squirrel heart and muscle}

To assess FoxOla mRNA levels in heart and muscle of ground squirrels, RT-PCR was performed using forward and reverse primers specific to FoxOla, as described by Cai et al. (2004). A 720 bp cDNA fragment was amplified in samples obtained from heart and skeletal muscle of euthermic and hibernating ground squirrels. Transcript levels of $\alpha$ tubulin, used as a housekeeping gene, were also assessed in the same samples; these generated an amplified fragment of 635 bp. Fig. 4.1A shows FoxOla mRNA expression in each organ together with expression levels of $\alpha$-tubulin. Transcript levels of FoxOla were normalized against $\alpha$-tubulin and the relative RNA expression levels were plotted as the ratio hibernation:euthermia (Fig. 4.1B). Transcript levels of FoxOla increased significantly by $2.02 \pm 0.05$ fold $(\mathrm{P}<0.01)$ in heart of hibernating squirrels compared with euthermic controls but remained stable in skeletal muscle $(0.99 \pm 0.05)$ during torpor. This indicates that regulation of FoxOla in heart, in contrast to muscle, may occur at the transcriptional level during hibernation.

\section{Western Blotting of FoxO1a and pFoxO1a (Ser 256)}

Western blotting assessed total FoxOla protein and the amount of phosphorylated pFoxOla (Ser 256) in ground squirrel heart and muscle using rabbit polyclonal 
antibodies. On one-dimensional SDS-PAGE gels, both tissues showed a single band, corresponding to the known molecular mass of FoxOla (69 kDa) and pFoxO1a (Ser 256) (75 kDa). Fig. 4.2A shows representative Western blots of FoxO1a and pFoxO1 (Ser 256) protein levels in heart and muscle of ground squirrels and Fig. 4.2B shows the mean ratios hibernating:euthermic for each protein, in each tissue. During hibernation, FoxO1a levels were significantly elevated in heart by $1.4 \pm 0.05$ fold $(\mathrm{P}<0.01)$ but remained unchanged between euthermic and hibernating muscle (ratio was $0.99 \pm 0.05$ ). These results are consistent with the mRNA transcript levels seen in Fig. 4.1B. Western blotting using antibodies that recognize the peptide surrounding the phosphorylation site (Ser256) on FoxOla showed that the amount of pFoxO1a increased significantly during hibernation. Mean ratios hibernating:euthermic for pFoxO1a (Ser 256) levels were $3.55 \pm$ 0.19 in heart $(\mathrm{P}<0.01)$ and $1.48 \pm 0.06(\mathrm{P}<0.05)$ in skeletal muscle.

\section{Distribution of FoxO1a and pFoxO1a in cytoplasmic and nuclear extracts from muscle}

Nuclear and cytoplasmic fractions were isolated from skeletal muscle of both euthermic and hibernating ground squirrels and then the distributions of FoxO1a and pFoxOla (Ser 256) were assessed in the two fractions using Western blotting. Fig. 4.3A shows a representative Western blot of FoxO1a in subcellular fractions of muscle. The results show that FoxOla is present almost exclusively in the nuclear fraction of both euthermic and hibernating samples. Similar levels of nuclear FoxOla expression occurred in both states. No band of FoxO1a was detected in the cytoplasm in either euthermic or hibernating animals. Fig 4.3B shows similar data using pFoxO1a (Ser 256). 
Again, there were no significant changes in the distribution between extracts from euthermic and hibernating animals. However, all the signals detected for pFoxOla (Ser 256) were located in the cytoplasmic fractions and no signal was seen in the nucleus in either state.

\section{FoxO1a DNA binding activity in ground squirrel tissues}

Transcription factors act by binding to DNA and their transcriptional activity depends on their protein concentration and the amount of binding to the DNA. To determine the effects of hibernation on the transcriptional activity of FoxO1a, changes in DNA binding activity were assessed in nuclear extracts from heart and skeletal muscle of both euthermic and hibernating ground squirrels using TransAM FKHR (FoxO1) kits. These ELISA-based kits contain the Forkhead Box (FOX) nucleotide element immobilized in the wells of 96-well microplates. Samples containing FoxOla transcription factor are added to the wells, binding to the FOX element occurs, and then the amount of binding is quantified as absorbance at $450 \mathrm{~nm}$. Fig. 4.4A shows the O.D. $460 \mathrm{~nm}$ for nuclear extracts of heart and muscle from euthermic versus hibernating squirrels. The mean values were $0.638 \pm 0.03$ and $0.679 \pm 0.04$ for heart from euthermic and hibernating animals, respectively. Binding activity was lower overall in skeletal muscle but a strong, significant reduction in binding was seen between euthermia $(0.337$ $\pm 0.02)$ and hibernation $(0.082 \pm 0.003)(\mathrm{P}<0.01)$. Expressed as the hibernating:euthermic ratio, it is clear that there was no significant change in DNA binding by FoxOla in heart during hibernation (ratio was $1.07 \pm 0.11, \mathrm{n}=3$ independent trials) whereas DNA binding activity significantly decreased (ratio was $0.24 \pm 0.02, \mathrm{P}<0.01, \mathrm{n}=3$ independent trials) 
in skeletal muscle during hibernation (Fig. 4.4B).

\section{Western Blotting of MAFbx, Bcl-3 and NFkB p50}

MAFbx protein was assessed via Western blotting in heart and skeletal muscle from euthermic and hibernating ground squirrels. The polyclonal antibodies used crossreacted with a protein band on the blot at $\sim 47 \mathrm{kDa}$, the known molecular weight of the MAFbx protein (Fig. 4.5A). In heart, levels of MAFbx did not change between euthermic and hibernating states (hibernating:euthermic ratio was $1.16 \pm 0.02$ ) (Fig. 4.5B). However, MAFbx protein levels decreased significantly in muscle during hibernation resulting in a hibernating:euthermic ratio of $0.82 \pm 0.01(\mathrm{P}<0.01)$.

Fig. 4.6A shows corresponding data for Bcl-3. Immunoblotting with polyclonal antibodies detected a protein band at $\sim 60 \mathrm{kDa}$ corresponding to the known molecular weight of Bcl-3. Bcl-3 protein levels increased significantly by $1.52 \pm 0.03$ fold in heart and $1.89 \pm 0.01$ fold in muscle of hibernating squirrels, compared with controls $(\mathrm{P}<0.01$ $\mathrm{n}=3$ independent trials for both tissues) (Fig. 4.6B).

NFKB p50 protein was assessed via Western blotting in heart and skeletal muscle of euthermic and hibernating ground squirrels. The polyclonal antibodies crossreacted with a protein band on the blot at $\sim 50 \mathrm{kDa}$, the known molecular weight of the $\mathrm{NF \kappa B}$ p50 protein (Fig. 4.7A). The amount of NFKB p50 was significantly higher in heart during hibernation (hibernating:euthermic ratio was $1.36 \pm 0.03, \mathrm{P}<0.01$ ) (Fig. 4.7B) whereas no significant differences were seen between euthermic and hibernating states in skeletal muscle (ratio was $1.08 \pm 0.02$ ). 


\section{Distribution of $\mathrm{NfKB}$ p50 and Bcl-3 in cytoplasmic and nuclear fractions}

The distribution of NFkB p50 in cytoplasmic and nuclear fractions isolated from heart and skeletal muscle of euthermic and hibernating ground squirrels was assessed by Western blotting. Fig. 4.8A shows representative Western blots of NfkB p50 in subcellular fractions of both heart and muscle. The results show a similar expression pattern of NFKB p50 in subcellular fractions of the two tissues. In both heart and muscle, NfkB p50 levels increased significantly by $1.67 \pm 0.03$ fold and $1.95 \pm 0.08$ fold (both $\mathrm{P}<0.01$ ), respectively, in cytoplasmic extracts during hibernation as compared with euthermia (Fig. 4.8B). However, in nuclear extracts from both tissues, NfkB p50 levels decreased significantly during hibernation with hibernating:euthermic protein ratios of $0.57 \pm 0.03$ in heart $(\mathrm{P}<0.05)$ and $0.43 \pm 0.06$ in muscle $(\mathrm{P}<0.01)$

The distribution of Bcl-3 was similarly evaluated but for heart only. The amount of $\mathrm{Bcl}-3$ in the cytoplasmic fraction increased by 1.29 -fold $(\mathrm{P}<0.01)$ during hibernation but remained constant in the nuclear fraction during hibernation (Fig. 4.9). These results indicate that $\mathrm{Bcl}-3$ did not translocate into the nucleus to transactivate downstream genes during hibernation.

\section{DISCUSSION}

FoxO1a, also called Forkhead in rhabdomyosarcoma (FKHR) transcription factor, is controlled by AKT (protein kinase B) signalling and is involved in regulating cell cycle progression, differentiation, proliferation, metabolism and apoptosis (Birkenkamp and Coffer, 2003; Schmidt et al., 2002). Recently, Sandri et al. (2004) have also reported the involvement of FoxOla in muscle atrophy. FoxOla is a substrate of AKT (Brunet et al., 
1999), carrying three AKT phosphorylation sites corresponding to Thr24, Ser256 and Ser319 in human FoxO1 (Barthel et al., 2005). It was previously shown in studies from our laboratory and others that the amount of phosphorylated AKT was significantly reduced in selected organs of thirteen-lined ground squirrels and bats (Myotis lucifugus) during hibernation (Eddy and Storey, 2003 - bats only; Cai et al. 2004b). In 13-lined ground squirrels this included brain, heart and skeletal muscle and, in addition, measured AKT activity in brain (the only tissue tested) was also reduced during hibernation consistent with the reduced amount of the active phosphorylated form of AKT (Cai et al., 2004b). The recent establishment of the link between the PI3K (phosphoinositide 3kinase)/AKT pathway, FOXO transcription factors and muscle atrophy, and the fact that ground squirrels spend several months during the hibernation season with very little physical activity or use of their muscles suggested that this natural model hibernator would be a good model in which to study aspects of disuse atrophy of heart and muscle. My studies began with an investigation of both transcript and protein levels of the Akt downsteam substrate, FoxO1a, in ground squirrel heart and skeletal muscle.

Relative RT-PCR and Western blots data showed that mRNA transcript levels and protein levels of FoxOla were differently regulated in the two tissues of hibernating ground squirrels. Using forward and reverse primers for FoxOla designed by Cai et al. (2004a), results for RT-PCR showed higher levels (2.02 fold) of FoxOla transcripts in heart from hibernating, as compared to euthermic, animals whereas no significant differences between the two states were observed in skeletal muscle (Fig. 4.1B). Western blot analysis indicated a similar pattern of expression, with a higher amount of FoxO1a protein in hibernating heart ( 1.4 fold) and no significant differences between euthermic 
and hibernating states in skeletal muscle (Fig. 4.2B). The increase in both FoxOla transcripts and FoxO1a protein in heart of hibernating squirrels indicates that the protein changes were a result of transcriptional activation of the FoxOla gene. However, FoxOla protein in both heart and muscle is also regulated at a post-translational level as evidenced by the strong increase in the amount of phosphorylated FoxOla (Ser256) seen during hibernation, a 3.55 fold increase in heart and 1.48 fold in muscle (Fig. 4.2B). These data confirm the findings of Cai et al. (2004a) who also reported that tissues from hibernating squirrels contained more of the phosphorylated form of FoxO1a as compared with euthermic animals. Next, the subcellular localization of FoxO1a and pFoxO1a (Ser256) in muscle of euthermic and hibernating ground squirrels was assessed. Recent observations by Tran et al. (2003) indicated that phosphorylation of FoxO1 on Ser256 leads to its interaction with 14-3-3 proteins and sequestration in the cytoplasm where the protein may remain complexed with $14-3-3$ proteins or is ubiquitinylated and then degraded. Consistent with this, our results showed that all of the unphosphorylated FoxO1a was localized inside the nucleus whereas phosphorylated FoxOla (Ser256) was all found in cytoplasmic extracts (Fig. 4.3). The increased levels of phosphorylated FoxOla (Ser256) when compared with the reduced amounts of active phospho-AKT in ground squirrel heart and muscle (Cai et al., 2004b) suggests that FoxO1a regulation in hibernation may not be under the control of the upstream kinase, AKT. Thus, other kinases may be involved in the post-translational modification of FoxOla during torpor. Indeed, it has been reported that several signal transduction pathways can regulate the function of FOXO. These include the serum and glucocorticoid-induced kinase (SGK) (Kops and Burgering, 2000), casein kinase (CK1) and the dual-specificity tyrosine- 
phosphorylated and regulated kinase 1A (DYRK1A) (Rena et al., 2002; Woods et al., 2001). Moreover, other factors that could also influence the amount of phosphorylated FoxO1a include the overall balance between kinase and phosphatase activities during hibernation; hence, a reduced level of AKT activity in torpor might be combined with an even greater reduction in the opposing phosphatase activity (Cai et al., 2004a). It will be interesting to further study the possible connections between FoxOla and AKT activity, and the role of their interaction in hibernation.

The DNA binding activity of FoxOla was also assessed in nuclear extracts of heart and muscle. There was no change in FoxOla DNA binding activity between euthermia and torpor in heart. However, FoxO1 a DNA binding activity strongly decreased in muscle during hibernation to just $24 \%$ of the euthermic value (Fig. 4.4B). In heart, the differential between the unchanged DNA binding activity ( 1.07 fold) and the 1.4 fold increase in FoxO1a protein levels, suggests the involvement of a mechanism of FoxO1a deactivation that may be protein phosphorylation. Despite the higher amount of the active FoxO1a protein, translated from the up-regulated Foxo1 a mRNA during torpor, the DNA binding activity remained unchanged. Since the content of pFoxO1a Ser 256 was elevated in hibernation, this suggests that FoxO1 a protein was being converted into the phosphorylated form during torpor, sequestered in the cytoplasm, and hence DNA binding by FoxO1a remained unchanged during torpor. Similarly, in skeletal muscle, the differential between the strongly decreased DNA binding activity ( 0.24 fold) and the unchanged FoxOla protein levels ( 0.99 fold) in muscle, agreed with the idea that protein phosphorylation inactivated FoxOla during torpor since higher levels of phosphorylated FoxO1a (Ser256) were found in that tissue (Fig 4. 2B). Moreover, Fig. 4.4B shows that 
pFoxO1a (Ser256) was exported from the nucleus to the cytoplasm (muscle was the only tissue tested) and supports the idea that phosphorylation may be the mechanism by which FoxOla was deactivated during torpor. This is in agreement with Van Der Heide et al. (2004), who reported that the phosphorylation of a specific serine residue in the nuclear localization sequence (NLS) motif present in FOXO protein is responsible for the shuttling mechanism from the nucleus to the cytosol, therefore terminating FOXO transcriptional function. In FoxO1a, the phosphorylation of Ser256 is thought to play a role in masking the NLS, thereby regulating transcriptional activity (Rena et al., 2001). FOXO transcription factors have been associated with the regulation of multiple biological processes, including the transcription of downstream targets genes in cellular functions such as metabolism, cell cycle, cell death, DNA repair and protection from oxidative stress (Van Der Heide et al., 2004). However, recent studies have also provided evidence that FoxOl transcription factors induce the atrophy-related ubiquitin ligase atrogin-1 (MAFbx), as well as MuRF1 (Muscle Ring Finger 1), thereby leading to skeletal muscle atrophy (Sandri et al., 2004; Kamei et al., 2004; Stitt et al., 2004). Levels of MAFbx protein were assessed in heart and muscle of ground squirrels. Western blots (Fig. 4.5B) revealed no significant change between euthermic and hibernating states in heart whereas the amount of MAFbx protein decreased by $18 \%$ in muscle of hibernating animals. These results neither indicate the occurrence of muscle atrophy in these organs nor support the contribution of MAFbx to muscle atrophy. Moreover, recent findings of Rourke et al. (2004) demonstrated that, in golden-mantled ground squirrels (S. lateralis), the expression of MAFbx mRNA was unchanged in the soleus muscle during torpor despite the fact that it was undergoing atrophy. Therefore, it seems likely that additional 
factors must be involved, potentially involving proteins such as MuRF1, Mdm2 and Nedd4 that have previously been shown to be involved in ubiquitination and muscle protein degradation (Jackman and Kandarian, 2004). Indeed, a study by Stitt et al. (2004) has also demonstrated that overexpression of MAFbx/atrogin-1 alone does not cause myotube or muscle atrophy.

Further work has implicated a new signaling pathway in disuse atrophy involving members of the NFKB and inhibitory $\kappa \mathrm{B}(\mathrm{I} \kappa \mathrm{B})$ family of transcriptional regulators: $\mathrm{p} 50$ and Bcl-3 (Hunter et al., 2002). A recent study established that muscular inactivity triggers the activation of $\mathrm{Bcl}-3$ and p50 which in turn activate genes involved in the induction of muscle atrophy (Hunter and Kandarian, 2004). Investigation of Bcl-3 protein levels in heart and skeletal muscle of ground squirrels revealed a higher content of Bcl-3 in both tissues of hibernating squirrels compared to the euthermic animals (Fig. 4.6B). This result led us to assess the amount of NFKB p50 in the same tissues since the evidence presented by Hunter and Kandarian (2004) suggested an association of muscle disuse atrophy with activation of NFKB through activation of p50 in complex with $\mathrm{Bcl}-3$. The protein levels of NFKB p50 in heart and skeletal muscle of euthermic and hibernating thirteen-lined ground squirrels showed different patterns. NFkB p50 protein rose in hibernating heart (1.38 fold) but was unchanged in skeletal muscle (Fig. 4.7B). Higher levels of both Bcl-3 and NFKB p50 in heart of hibernating animals suggests the possible formation of a transcriptional-complex that may activate downstream genes. In contrast, no correlation was observed between these two proteins in skeletal muscle. However, it is the nuclear content of NFKB p50 that is critical to gene activation and the analysis of $\mathrm{NF \kappa B}$ p50 levels in cytoplasmic versus nuclear extracts is key for assessing the potential 
for transcriptional activation in euthermic versus hibernating states. The data showed higher amounts of NFKB p50 in the cytoplasmic fractions of both tissues during hibernation, 1.67 fold and 1.95 fold increases (Fig. 4.8B). By contrast, NFkB p50 content in nuclear fractions was reduced during hibernation to $57 \%$ and $43 \%$ of the euthermic values in hibernating heart and muscle, respectively. This suggests that even though elevated levels NFKB p50 (in heart) and Bcl-3 (in both tissues) could support an increase in the level of the complex between these two proteins that can activate genes, the potential amount of this complex in the nucleus (assessed by NFKB p50 content) actually decreased during hibernation. Hence, it is unlikely that a NFKB p50 / Bcl-3 complex could be involved in the transcriptional activation of downstream genes that might be involved in muscle atrophy during torpor.

In summary, the results of the present study indicate that levels of phosphorylated FoxOla (Ser256) were significantly increased in both heart and skeletal muscle during hibernation, even though the FoxOla upstream kinase, AKT, was previously shown to be significantly suppressed during hibernation. This suggests the possibility that FoxOla may be controlled by a different upstream kinase during hibernation. Moreover, protein levels of one of the FoxOla downstream genes, MAFbx / atrogin 1, were unchanged or reduced during hibernation despite the fact that $\mathrm{MAFbx}$ mRNA was up-regulated in muscle during hibernation in a closely-related species, S. lateralis. However, elevated mRNA transcripts may not actually mean increased translation during torpor, a time when multiple indicators suggest that transcription is strongly suppressed (Storey and Storey, 2004). Transcripts may be maintained during torpor to support rapid protein synthesis when the animal enters an arousal phase. Although previous studies on ground squirrels 
(S. lateralis) demonstrated a slight atrophy of skeletal muscle, the results presented in this study on selected genes that are known to be involved in disuse atrophy in other systems shows down-regulation or inactivation of these during hibernation. This is consistent with either low atrophy in torpid animals or with the involvement of other regulatory mechanisms in mediating the disuse atrophy that does occur. In this respect, future studies should focus on a new signalling pathway identified recently by Glass (2005), the Inhibitor of nuclear factor $\kappa \mathrm{B}$ Kinase $(\mathrm{IKK}) / \mathrm{NF} \mathrm{KB} / \mathrm{MuRF} 1$, which induces muscle atrophy and protein breakdown via the up-regulation of the E3 ubiquitin-ligase MuRF1. 
Fig. 4.1: Analysis of FoxOla transcript levels in ground squirrel heart and skeletal muscle. (A) Representative RT-PCR blots showing FoxOla and alpha-tubulin transcript levels in tissue extracts from euthermic (E) and hibernating $(\mathrm{H})$ animals. (B) Bar graphs showing the mean ratio of FoxOla mRNA levels (relative to $\alpha$-tubulin) in the two muscle types. The lowest dilutions for alpha-tubulin and FoxOla which have visible PCR products were chosen for quantification; these were typically $10^{-3}-10^{-4}$ dilution. Statistical analysis of normalized values from euthermic versus hibernating samples used the Student's $t$-test with significance levels $(\mathrm{P}<0.01)$. The mean ratios of hibernating to euthermic values and the S.E.M were then calculated and plotted in histograms, with the S.E.M on the ratios representing the error bar on the histograms. ** - Values for the hibernating condition are significantly different from the corresponding euthermic values, $\mathrm{P}<0.01, \mathrm{n}=3$ independent trials with both hibernating and euthermic samples. 
Fig. 4.1

A.

\begin{tabular}{|c|c|c|c|c|}
\hline & \multicolumn{2}{|c|}{ Heart } & \multicolumn{2}{|c|}{ Muscle } \\
\hline & $\mathrm{E}$ & $\mathrm{H}$ & $\mathrm{E}$ & $\mathrm{H}$ \\
\hline FOXO1a & 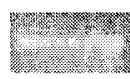 & Fis & 2 & pas \\
\hline -Tubu & rese & Ses. & & \\
\hline
\end{tabular}

B.

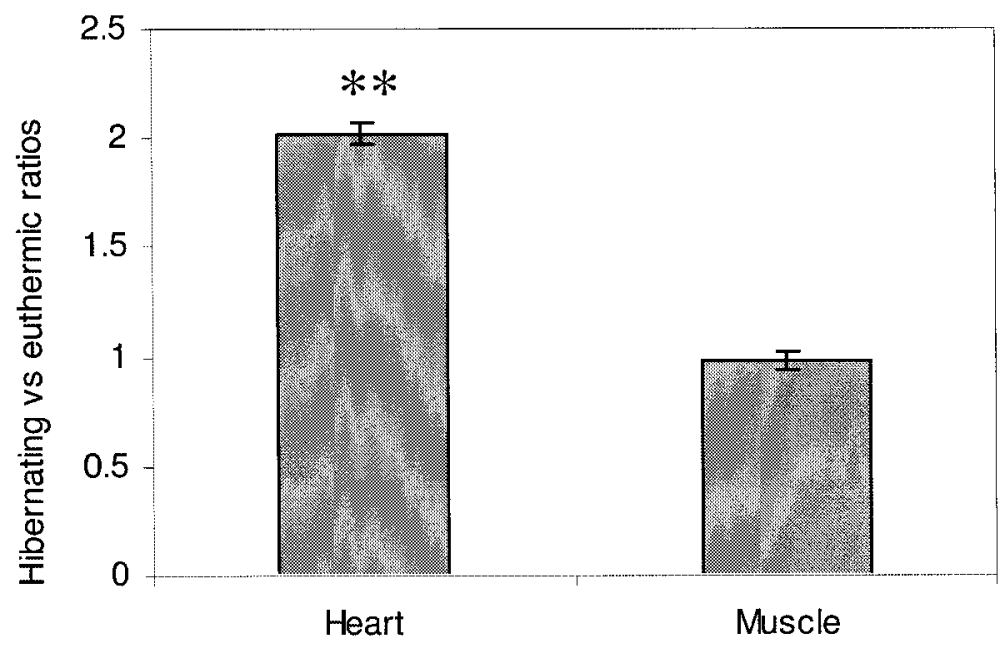


Fig. 4.2: FoxOla and pFoxO1a Ser 256 protein expression in muscle and heart of thirteen-lined ground squirrels. (A) Representative Western blots showing the FoxOla protein at $\sim 69 \mathrm{kDa}$ and $\mathrm{pFoxO}$ a Ser 256 protein band at $\sim 75 \mathrm{kDa}$ in euthermic $(\mathrm{E})$ and hibernating $(\mathrm{H})$ ground squirrels. (B) Histograms showing the relative FoxOla protein and pFoxO1a Ser 256 protein levels in ground squirrels organs. Data are means \pm S.E.M. * and ** - Values for hibernating samples are significantly different from the corresponding euthermic values using the two-tailed Student's $t$-test, $\mathrm{P}<0.05$ and $\mathrm{P}<$ 0.01 respectively, $n=3$ trials on tissue from different animals. 
Fig. 4.2

A.

\section{FoxOla}

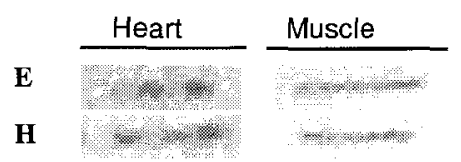

pFoxO1a Ser256

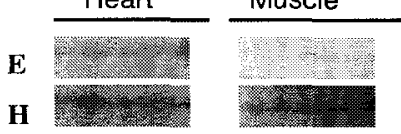

B.

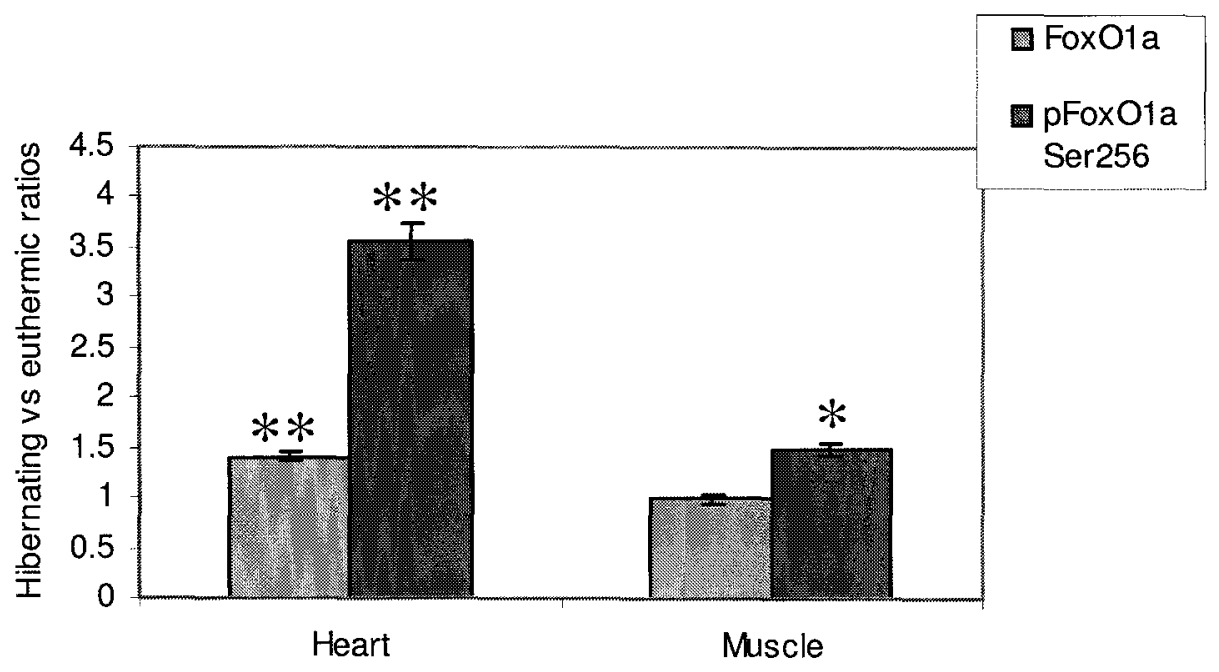


Fig. 4.3: Distribution of FoxOla and pFoxOla between cytoplasmic and nuclear fractions in skeletal muscle of $S$. tridecemlineatus. Representative Western blots $(\mathrm{n}=3$ trials) show strong expression of the FoxO1a protein band at $\sim 69 \mathrm{kDa}(\mathbf{A})$ in nuclear extracts whereas the pFoxO1a protein band at $75 \mathrm{kDa}(\mathbf{B})$ dominates in the cytoplasmic extract from skeletal muscle of both euthermic $(\mathrm{E})$ and hibernating $(\mathrm{H})$ ground squirrels. 
Fig. 4.3

A.

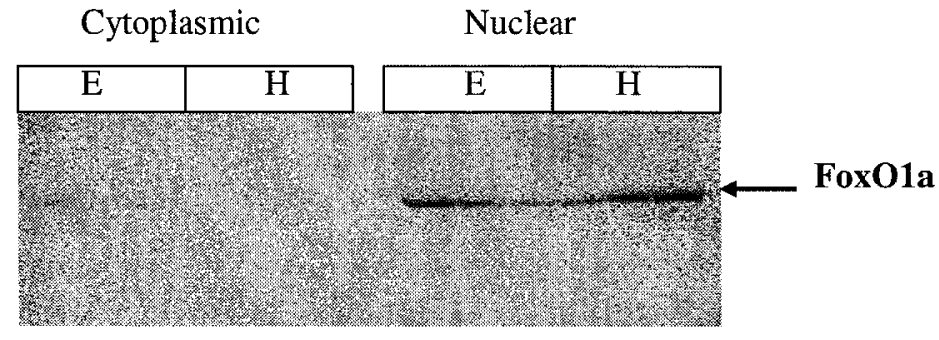

B.

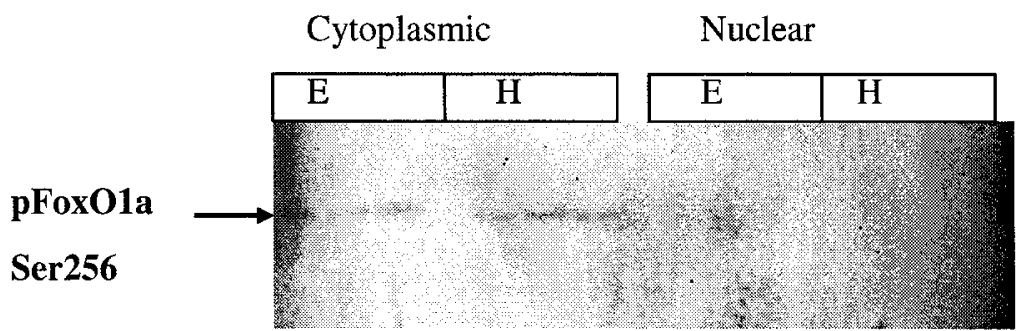


Fig. 4.4: FoxO1a DNA binding activity in heart and skeletal muscle of euthermic and hibernating thirteen-lined ground squirrels. (A) Binding assays of FoxO1a to its consensus-binding site were performed according to the manufacturer's protocol and absorbance of the bound FoxO1a measured at $460 \mathrm{~nm}$. (B) Histograms show the ratio hibernating: euthermic FoxO1a levels. Data are mean \pm SEM for $n=3$ independent trials. ** - Hibernating values are significantly different from the corresponding euthermic values, $\mathrm{P}<0.01, \mathrm{n}=3$ trials on tissue from different animals. 
Fig. 4.4

A.

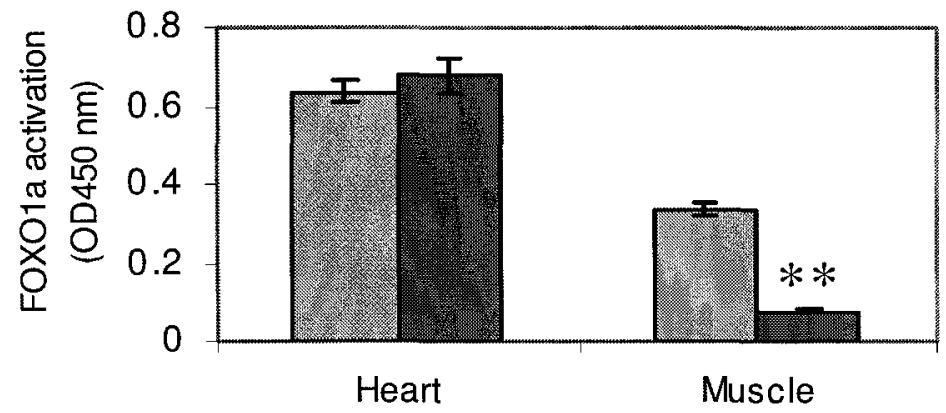

Euthermic

Hibernating

B.

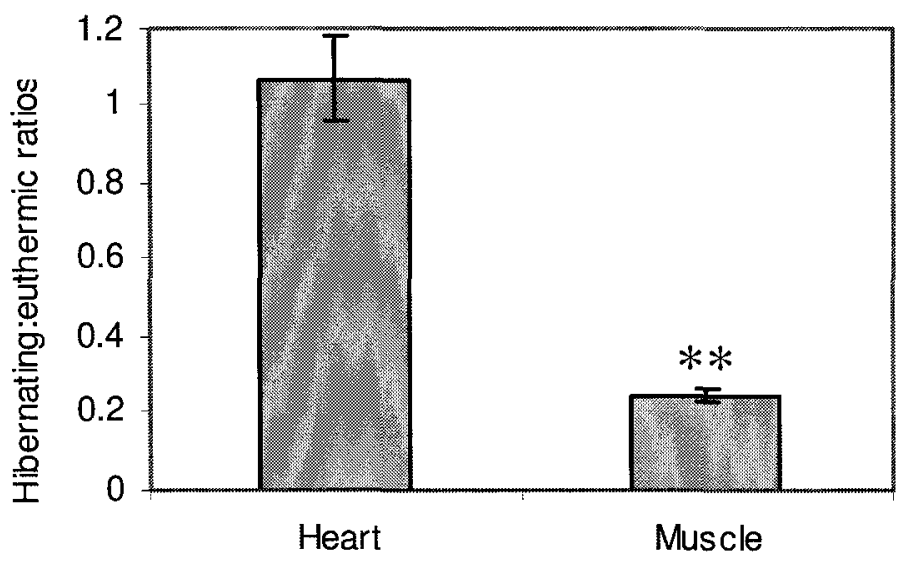


Fig. 4.5: MAFbx / atrogin-1 protein expression in heart and muscle of thirteen-lined ground squirrels. (A) Representative Western blots showing MAFbx protein band at $\sim 47$ $\mathrm{kDa}$ in organs of euthermic (E) and hibernating (H) ground squirrels. (B) Histograms showing the relative MAFbx protein levels in ground squirrels organs. Data are means \pm S.E.M., $\mathrm{n}=3$ independent trials. $* *$ - Values for hibernating samples are significantly different from the corresponding euthermic values using the two-tailed Student's $t$-test, $\mathrm{P}$ $<0.01, \mathrm{n}=3$ trials on tissue from different animals. 
Fig. 4.5

A.

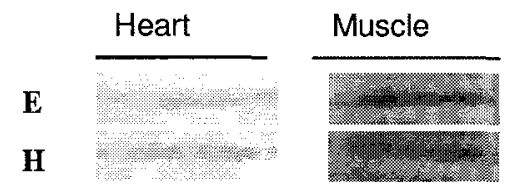

B.

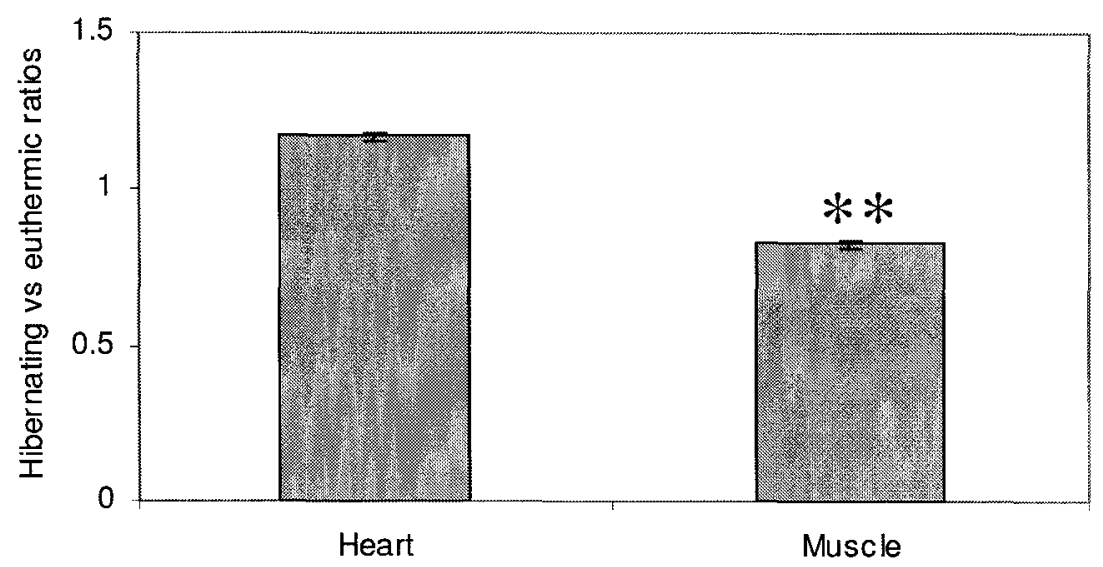


Fig. 4.6: Bcl-3 protein expression in heart and muscle of thirteen-lined ground squirrels. (A) Representative Western blots showing Bcl-3 protein band at $\sim 60 \mathrm{kDa}$ in organs of euthermic (E) and hibernating $(\mathrm{H})$ ground squirrels. (B) Histograms showing the relative Bcl-3 protein levels in ground squirrels organs. Data are means \pm S.E.M., $n=3$ independent trials. ** - Values for hibernating samples are significantly different from the corresponding euthermic values using the two-tailed Student's $t$-test, $\mathrm{P}<0.01, \mathrm{n}=3$ trials on tissue from different animals. 
Fig. 4.6

A.

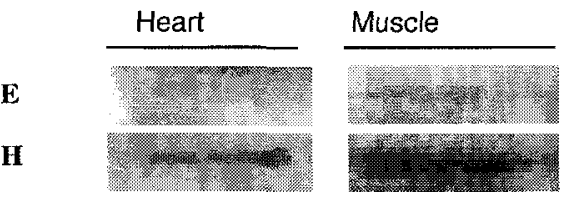

B.

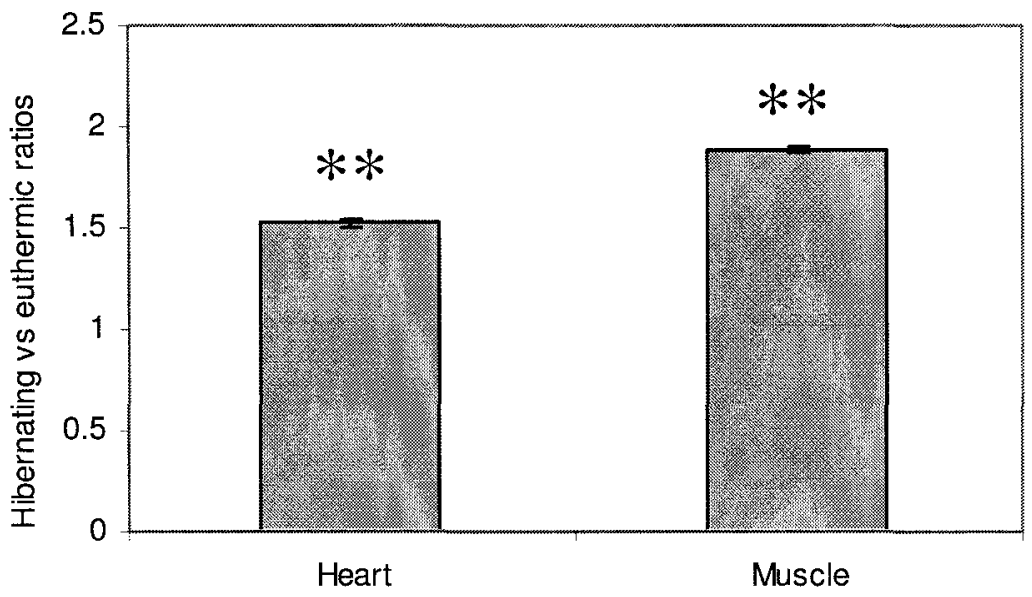


Fig. 4.7: NFKB p50 protein expression in heart and muscle of thirteen-lined ground squirrels. (A) Representative Western blots showing NFkB p50 protein band at $\sim 60 \mathrm{kDa}$ in organs of euthermic $(\mathrm{E})$ and hibernating $(\mathrm{H})$ ground squirrels. (B) Histograms showing the relative NFKB p50 protein levels in ground squirrels organs. Data are means \pm S.E.M., $\mathrm{n}=3$ independent trials. ** - Values for hibernating samples is significantly different from the corresponding euthermic values using the two-tailed Student's $t$-test, $\mathrm{P}$ $<0.01, \mathrm{n}=3$ trials on tissue from different animals. 
Fig. 4.7

A.

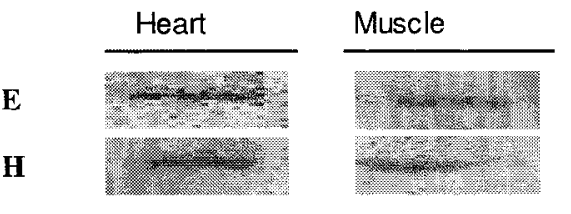

B.

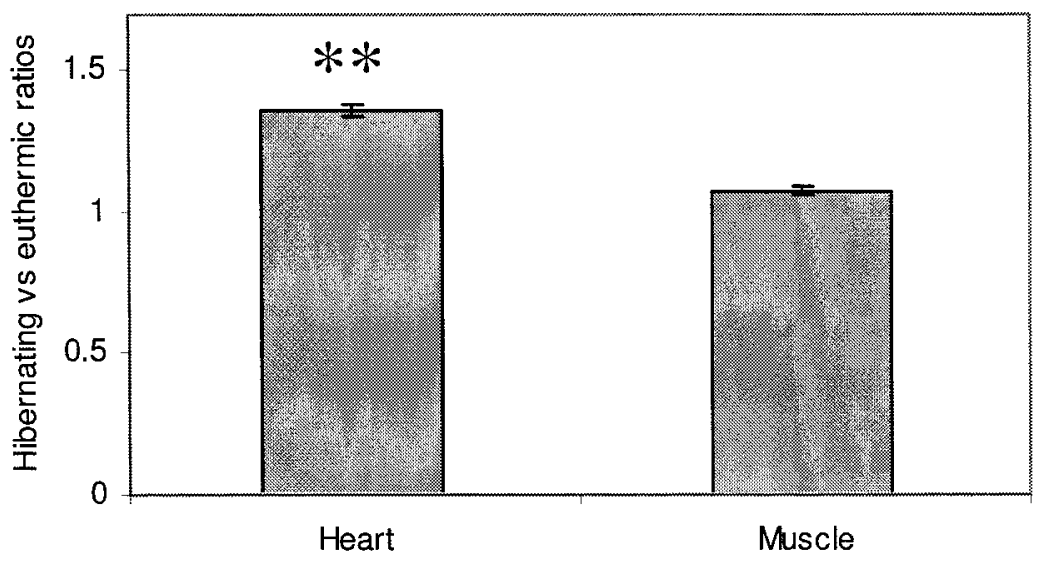


Fig. 4.8: Analysis of NFKB p50 protein distribution between cytoplasmic and nuclear fractions in heart and skeletal muscle of euthermic $(E)$ and hibernating $(H)$ ground squirrels. (A) Representative Western blots showing detection of p50 in nuclear and cytoplasmic extracts. (B) Histograms showing relative levels of NFKB p50 in cytoplasmic and nuclear fractions from each tissue. Data are means \pm SEM, $n=3$ independent determinations. $* * *$ - Values for hibernating sample are significantly different from the corresponding euthermic values, $\mathrm{P}<0.05$ and $\mathrm{P}<0.01$, respectively. 
Fig. 4.8

A.

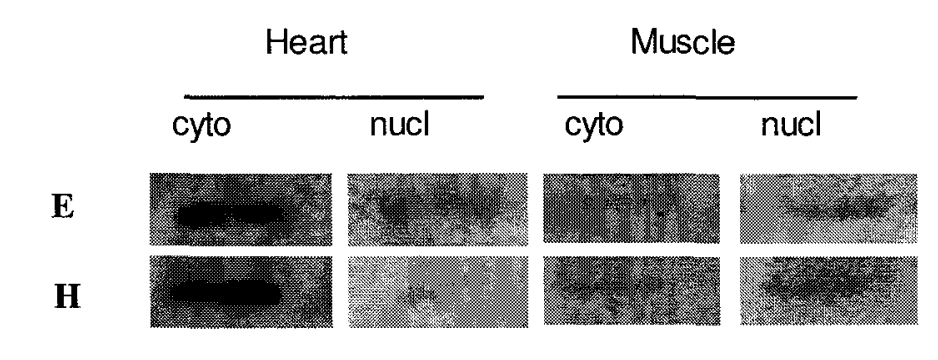

B.

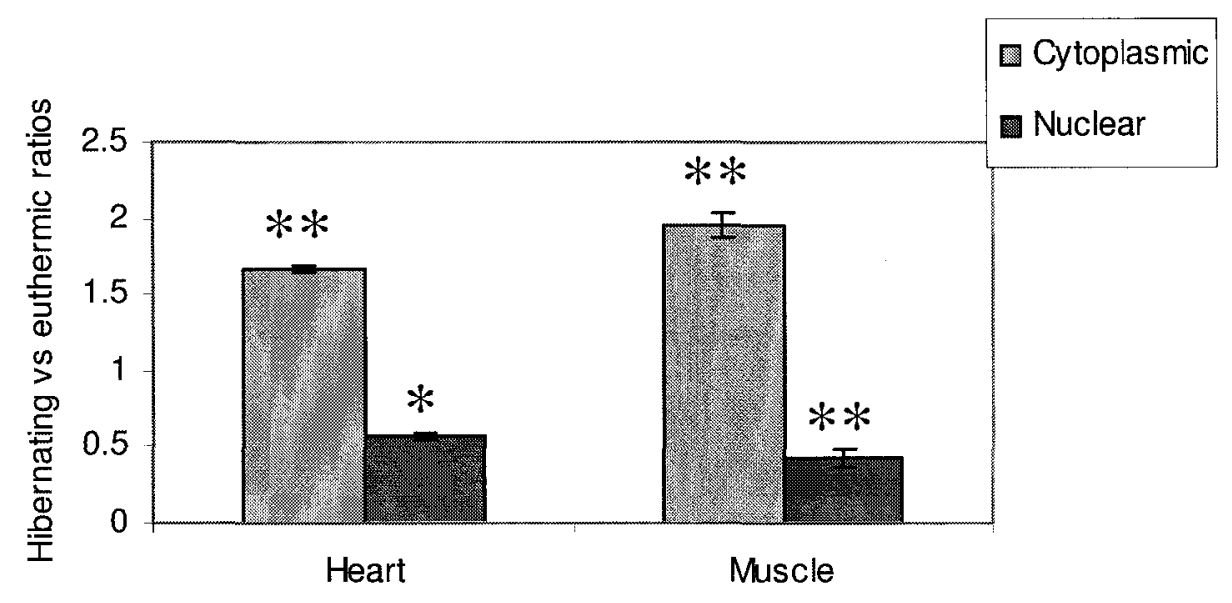


Fig. 4.9: Analysis of $\mathrm{Bcl}-3$ protein distribution between cytoplasmic and nuclear fractions in heart of euthermic (E) and hibernating (H) ground squirrels. (A) Representative Western blots showing detection of Bcl-3 in nuclear and cytoplasmic extracts. (B) Histograms showing relative levels of Bcl-3 in cytoplasmic and nuclear fractions from heart. Data are means \pm SEM, $n=3$ independent determinations. ** Values for hibernating sample are significantly different from the corresponding euthermic values, $\mathrm{P}<0.01, \mathrm{n}=3$ trials on tissue from different animals. 
Fig. 4.9

A.

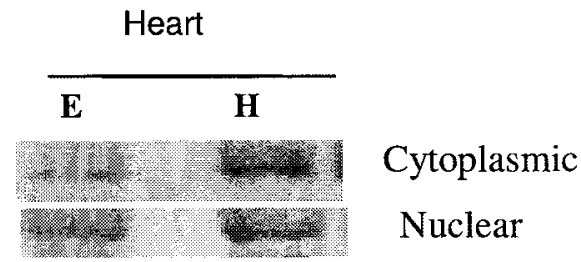

B.

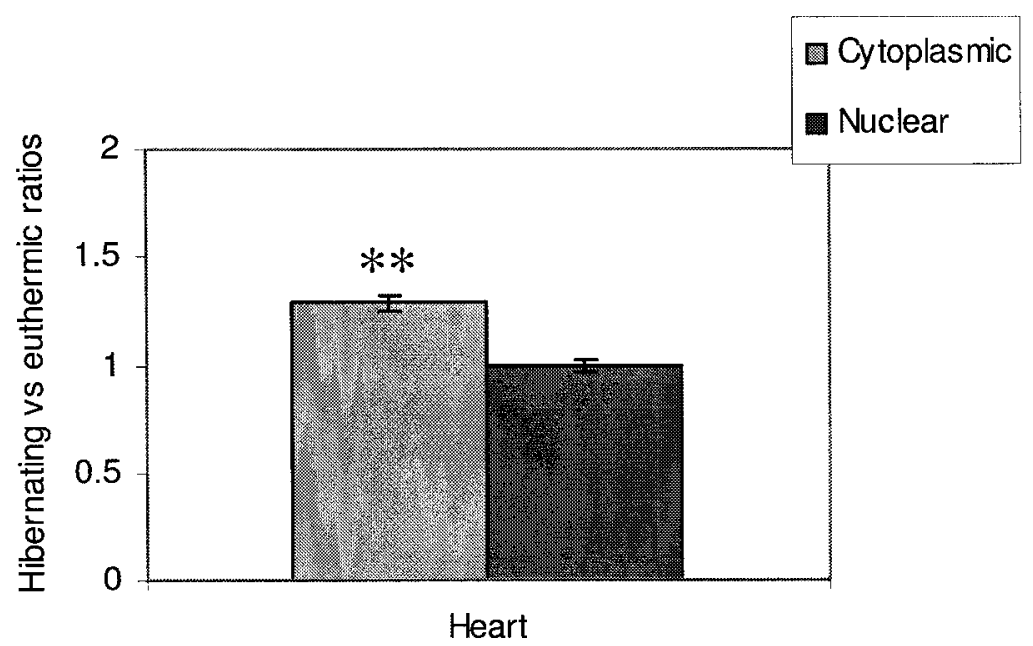




\title{
Chapter 5
}

\section{Bcl-2 family members and anti-apoptotic defense}

\author{
in hibernating ground squirrels
}




\section{INTRODUCTION}

Apoptosis, or programmed cell death, is an active and complex process in which cells respond to internal and/or external stimuli, by triggering a cell-suicide program (Chang et al., 1997). Upon apoptotic stimulation, cells that receive the apoptosistriggering stimuli enters a degradation phase, which is characterized by a number of morphological and biochemical changes including DNA fragmentation and degradation of multiple structural and repair proteins in the cytosol, cytoskeleton, and nucleus (Zamzami et al., 1998). A variety of physiological death signals and pathological cellular insults trigger the genetically programmed pathway of apoptosis (Vaux and Korsmeyer, 1999). According to Ciriolo (2005), apoptosis is a genetically encoded form of cell death, central to the development and homeostasis of multicellular organisms and designed to rapidly remove unwanted surplus cells or potentially dangerous cells. However, the inappropriate regulation of apoptosis is associated with a variety of diseases including cancer, neurodegenerative diseases and ischemic stroke (Strasser et al., 2000). Environmental stresses including oxidative stress and hypoxia/anoxia can also initiate apoptosis in cells by triggering DNA degradation and release of cytochrome $c$ from mitochondria, leading to cellular damage and organ dysfunction (Noda et al., 1998; Fleck and Carey, 2005).

Moreover, it has been reported that apoptosis proceeds through two main pathways, regulated at multiple levels: the extrinsic apoptotic pathway involving caspases and the intrinsic pathway centered on the mitochondria (Gross et al., 1999; Ciriolo, 2005). The intrinsic pathway involves the Bcl-2 (B cell leukemia/lymphoma protein 2) family of proteins that may have anti-apoptotic function (such as Bcl-XL, Bcl-2 and 
MCL-1) or pro-apoptotic function (such as Bax, Bak and BAD). In addition, the ratio between the anti-apoptotic proteins (antagonists) and the pro-apoptotic proteins (agonists) dictates or determines the susceptibility of a cell to a death signal (Gross et al. 1999; Zha et al., 1996). Indeed, Fleck and Carey (2005) have provided evidence that enhanced expression of anti-apoptotic proteins ( $\mathrm{Bcl}-\mathrm{XL}$ and $\mathrm{Bcl}-2)$ can limit apoptosis and promote enterocyte survival in intestine under the pro-oxidative and pro-apoptotic environment in hibernating mammals. Therefore, I proposed that modulation of the proteins in this family would be expected in other organs of thirteen-lined ground squirrels during hibernation.

Proteins in the Bcl-2 family contain four conserved homology domains (BH1, $\mathrm{BH} 2, \mathrm{BH} 3, \mathrm{BH} 4)$ except for BAD (Bcl-2-associated death protein) which lacks the BH4 domain. The missing BH4 domain is associated with anti-apoptotic function and, indeed, deletion of that domain converted the anti-apoptotic Bcl-2 protein into a pro-apoptotic protein (Huang et al., 1998). Bcl-XL and Bcl-2 proteins reside in the mitochondria outer membrane (Janumyan et al., 2003) and play an important role at this level in deciding whether a cell will live or die by controlling mitochondrial permeability and the release of cytochrome $c$. However, BAD resides in the cytosol and translocates to the mitochondria following death signaling. BAD heterodimerizes through its $\mathrm{BH} 3$ domain with $\mathrm{Bcl}-\mathrm{XL}$ and $\mathrm{Bcl}-2$ to block their pro-survival function and, thereby, promote apoptosis (Yang et al., 1995).

Recent studies have provided evidence that survival factors such as interleukin 3 (IL-3) inhibit the apoptotic activity of BAD by activating intracellular signaling pathways that result in the phosphorylation of $\mathrm{BAD}$ at serine residues 112 and 136 (Tan et al., 
2000). One of these pathways involved the phosphatidylinositol 3-kinase (PI3K) and its target, the protein kinase B (AKT) which has been shown to regulate apoptosis by phosphorylating BAD at serine 136. Another pathway involves IL-3 and protein kinase A (PKA) that phosphorylates BAD at serine 112 (Tan et al., 2000; Zha et al., 1996). Phosphorylation of BAD at these two sites results in its binding to 14-3-3 proteins and sequesters phosphorylated BAD in the cytosol which prevents cytotoxic interactions with Bcl-XL and Bcl-2 at the mitochondrial membrane (Zha et al., 1996). Moreover, it has been shown that phosphorylation of $\mathrm{BAD}$ at serine residue 155 by PKA directly blocks the $\mathrm{BH} 3$ dependent dimerization with $\mathrm{Bcl}-\mathrm{XL}$ and inhibits the death-promoting activity of BAD (Tan et al., 2000).

Evidence indicates that ground squirrel organs undergo physiological changes associated with oxidative stress and ischemia/reperfusion (Kurtz et al., 2006) over torporarousal cycles during hibernation and similar conditions are known to trigger apoptosis in nonhibernating mammals. Hence, I hypothesized that altered expression of the antiapoptotic proteins, $\mathrm{Bcl}-\mathrm{XL}$ and $\mathrm{Bcl}-2$, as well as the pro-apoptotic protein, $\mathrm{BAD}$, may be part of the adaptive strategy that provides protection of ground squirrel cells against apoptosis during hibernation.

The present chapter uses Western blotting to quantify the relative levels of proteins that have anti-apoptotic functions ( $\mathrm{Bcl}-\mathrm{XL}$ and $\mathrm{Bcl}-2)$ and examines changes in both total BAD protein content and the amounts of phospho-BAD Ser 112, Ser 136 and Ser155 in selected organs of euthermic versus hibernating thirteen-lined ground squirrels. A partial $b c l-x l$ cDNA sequence was amplified and used to quantify transcript levels by RT-PCR. The results provide evidence of the enhanced expression of anti-apoptotic 
proteins and the suppression or inhibition of pro-apoptotic proteins during torpor, and hence the importance of anti-apoptotic defense in hibernation to promote cell survival.

\section{MATERIALS AND METHODS}

Animals.

All animal experiments were as described in Chapter 2.

\section{RNA preparation}

Total RNA was isolated from three samples of each tissue (BAT, brain, heart, kidney, liver and skeletal muscle) from euthermic and hibernating animals using Trizol reagent (Invitrogen) as previously described in Chapter 2.

cDNA synthesis and PCR amplification of Bcl-XL.

First strand synthesis was performed as described previously (Chapter 2). The amplification of $b c l-x l$ via relative RT-PCR was performed as in Chapter 2 with primers: forward 5'- GGATACAGCTGGAGTC - 3' and reverse 5' GCAATCCGACTCACCAATAC - 3'. The cycling conditions for PCR were $95^{\circ} \mathrm{C}$ for 4 min, then 35 cycles of $95^{\circ} \mathrm{C}$ for $30 \mathrm{sec}, 57^{\circ} \mathrm{C}$ for $30 \mathrm{sec}$, and $72^{\circ} \mathrm{C}$ for $30 \mathrm{sec}$, followed by a final step at $72^{\circ} \mathrm{C}$ for $7 \mathrm{~min}$. A $b c l-x l$ fragment of $438 \mathrm{bp}$ was amplified and sequenced by CORTEC (Kingston, ON). The deduced amino acid sequence was confirmed to be Bcl-XL via the BLAST-P program.

\section{Western Blotting}

Western blotting was performed as described in Chapter 2. Total protein was isolated from samples of six tissues (BAT, brain, heart, kidney, liver and muscle) of 
euthermic and hibernating ground squirrels. Aliquots containing $10-30 \mu \mathrm{g}$ of protein were loaded into each well of $12 \%$ SDS gels, followed by electrophoresis, and then transfer to PVDF membrane. Rabbit polyclonal antibodies to Bcl-XL and Bcl-2 were purchased from Santa Cruz Biotechnologies. Rabbit polyclonal antibodies against BAD, phosphoBAD-Ser112, phospho-BAD-Ser136, phospho-BAD-Ser155 as well as HRP-linked antirabbit IgG (H\&L) secondary antibody were purchased from Cell Signaling Technologies. All primary antibody incubations for Western blotting were carried out at $4^{\circ} \mathrm{C}$ overnight, except for Bcl-2 (3 days), in 1X TBST at 1:300 v/v dilution for Bcl-XL and Bcl-2, 1:1000 $\mathrm{v} / \mathrm{v}$ dilution for BAD, pBAD-Ser112 and p-BAD-Ser 155, and 1:250 v/v dilution for pBAD-Ser136.

\section{RESULTS}

\section{cDNA cloning of $b c l$-xl from $S$. tridecemlineatus}

A consensus sequence of the $b c l-x l$ gene from human mouse and rat (accession numbers BT007208, X83574, and U34963, respectively) was used to design forward and reverse primers specific to $b c l$ - $x l$. These primers were then used in RT-PCR to amplify $b c l-x l$ cDNA from total RNA prepared from brain of thirteen-lined ground squirrels. A 438 bp cDNA fragment was amplified and nucleotide sequencing showed that it encoded a portion of $b c l-x l$; the sequence was submitted to GenBank with the accession number DQ503423. The nucleotide and deduced amino acid sequences are shown in Fig. 5.1. Translation of the ground squirrel $b c l-x l$ cDNA sequence into protein revealed a partial sequence of 146 amino acids, compared with the full length Bcl-XL sequence from human, mouse and rat that has 233 amino acids. Fig. 5.2 shows the alignment of the Bcl- 
$\mathrm{XL}$ sequence from ground squirrel with the sequences of the human, mouse and rat protein. Three amino acid substitutions were evident in the ground squirrel sequence as compared with the non-hibernating mammals. These substitutions were a glutamine residue at position 58 compared with histidine in other species, and an isoleucine substitution at position 65 replacing valine in the other sequences.

The gene and the protein are highly conserved among mammalian species. The homology tree shows that the ground squirrel $b c l-x l$ cDNA sequence was $97 \%$ identical to human and $92-95 \%$ identical to mouse and rat $b c l-x l$ sequences over the amplified region (Fig. 5.3A). The deduced amino acid sequence shared $98 \%$ identity to human Bcl$\mathrm{XL}$ and was $95-97 \%$ identical to mouse and rat Bcl-XL sequences (Fig. 5.3B).

\section{Transcript levels of $b c l-x l$ in thirteen-lined ground squirrel tissues}

Using a semi-quantitative RT-PCR and consensus primers derived from known mammalian $b c l-x l$ gene sequences, $b c l-x l$ mRNA transcript levels were assessed in six tissues (BAT, brain, heart, kidney, liver, skeletal muscle) of euthermic versus hibernating ground squirrels. Fig. 5.4A shows $b c l$ - $x l$ mRNA expression in each organ together with expression levels of $\alpha$-tubulin. Transcript levels of $b c l$ - $x l$ were normalized against $\alpha$ tubulin amplified from the same samples, expression ratios were calculated, and mean ratios in hibernation versus euthermia are shown in Fig. 5.4B. Transcript levels of $b c l-x l$ increased significantly by 1.83 -fold in heart and 1.50 -fold in kidney of hibernating squirrels compared with euthermic controls $(\mathrm{P}<0.05)$. Levels decreased significantly in BAT and skeletal muscle during hibernation, falling to $78 \%$ and $50 \%$ of the euthermic values, respectively (Fig. 5.4B). Transcript levels of $b c l$-xl remained unchanged in brain 
and liver during hibernation.

\section{Bcl-XL protein levels in thirteen-lined ground squirrel tissues}

Bcl-XL protein expression was assessed via Western blotting in six tissues of euthermic and hibernating ground squirrels. The polyclonal antibodies used crossreacted with a protein band on the blot at $30 \mathrm{kDa}$, the known molecular weight of the $\mathrm{Bcl}-\mathrm{XL}$ protein (Fig. 5.5A). Bcl-XL protein content rose significantly in four tissues during hibernation with hibernating:euthermic ratios of $1.50 \pm 0.03$ in BAT $(\mathrm{P}<0.01), 1.47 \pm$ 0.09 in heart $(\mathrm{P}<0.05), 1.56 \pm 0.08$ in kidney $(\mathrm{P}<0.05)$ and $1.41 \pm 0.05$ in muscle $(\mathrm{P}<$ 0.05) (Fig. 5.5B). Levels of Bcl-XL did not change between euthermic and hibernating states in brain and liver (hibernating:euthermic ratios were $1.06 \pm 0.06$ and $1.06 \pm 0.04$ respectively).

\section{Bcl-2 protein levels in $S$. tridecemlineatus}

Fig. 5.6A shows representative Western blots of $\mathrm{Bcl}-2$ protein levels in the six tissues of ground squirrels and Fig. 5.6B shows the mean ratios hibernating:euthermic. Bcl-2 content remained unchanged between euthermic and hibernating BAT (0.96 \pm 0.04). However, levels were very strongly elevated in brain by $4.68 \pm 0.10$ fold $(\mathrm{P}<0.05)$ during hibernation, and also rose significantly in heart by $2.02 \pm 0.06$ fold $(\mathrm{P}<0.05)$, in kidney by $2.72 \pm 0.13$ fold $(\mathrm{P}<0.01)$ and in liver by $2.15 \pm 0.02$ fold $(\mathrm{P}<0.05)$. Bcl-2 protein level decreased significantly in skeletal muscle during hibernation resulting in a hibernating:euthermic ratio of $0.52 \pm 0.06, \mathrm{P}<0.05$ (Fig. 5.6B). 


\section{BAD and pBAD (Ser 112, Ser 136 and Ser 155) protein levels}

Total BAD protein, as well as the amount of phospho-BAD phosphorylated on Ser 112, Ser 136 or Ser 155 , was measured by immunoblotting in extracts of heart and skeletal muscle from euthermic and hibernating ground squirrels (Fig. 5.7A and Fig. 5.8A). Each antibody crossreacted with a single protein band at $23 \mathrm{kDa}$. Fig. 5.7B shows that there was no significant change in the total amount of BAD protein in the two organs between hibernating and euthermic states (hibernating:euthermic ratios were $1.06 \pm 0.02$ and $0.96 \pm 0.03$, respectively.

However, the phosphorylation of BAD changed substantially during torpor. In heart, levels of all three phosphorylated forms of BAD increased significantly during hibernation, compared to euthermia. Antibodies detecting specific phosphopeptides showed a 1.92-fold $(\mathrm{P}<0.01)$ increase in the amount of phosphorylation at Ser 112 , a 1.29 -fold $(P<0.05)$ fold increase in pBAD Ser 136 , and a 2.66 -fold increase $(P<0.01)$ in pBAD Ser 155 (Fig. 5.8B). The opposite response was seen in skeletal muscle. Protein levels of pBAD Ser 136 and pBAD Ser 155 decreased significantly during hibernation resulting in a hibernating:euthermic ratio of $0.54 \pm 0.03$ and $0.48 \pm 0.02$, respectively ( $P$ $<0.01$ ) (Fig. 5.8B) whereas pBAD Ser 112 content did not change (hibernating:euthermic ratio was $1.01 \pm 0.03)$.

\section{DISCUSSION}

Features of the mammalian hibernation phenotype that might favor or affect apoptosis (programmed cell death) in nonhibernating species include hypothermia, oxidative stress (caused by rapid increases in oxygen consumption during arousal) (Carey 
et al., 2003b) and ischemia-reperfusion (Noda et al., 1998). A growing body of evidence has shown that over-expression of $\mathrm{Bcl}-2$ enhances survival of mammalian cells in response to various stimuli (Mastrangelo et al., 2000). Investigation of the regulation of apoptotic pathways in mammalian hibernators has just begun and the effect of hibernation on apoptosis is still poorly understood. Recently studies on the modulation of apoptotic pathways in intestinal mucosa of hibernating ground squirrels provided evidence for enhanced expression of anti-apoptotic proteins that may promote enterocyte survival during the hibernation period (Fleck and Carey, 2005).

Transcript levels of $b c l-x l$ were measured in six tissues of euthermic and hibernating 13-lined ground squirrels. In heart and kidney, increased levels of both $b c l-x l$ transcripts (1.5-1.83 fold) and Bcl-XL protein (1.5-fold) were found during hibernation (Fig. 5.4 and 5.5). The correlation between bcl-xl transcript and Bcl-XL protein levels in these two organs suggests the importance and implication of Bcl-XL in the anti-apoptotic response associated with hibernation. Bcl-XL protein levels were also increased significantly by $\sim 50 \%$ in BAT and skeletal muscle during hibernation although in these organs the protein increase was not supported by a corresponding elevation of $b c l-x l$ transcripts (indeed, $b c l-x l$ transcripts decreased in both cases). Brain and liver showed no significant changes in either $b c l-x l$ transcripts or Bcl-XL protein during hibernation. Hence, these date provide evidence of organ-specific needs for anti-apoptotic proteins during torpor. Increased Bcl-XL protein in BAT and skeletal muscle without elevated $b c l$ $x l$ transcripts suggests that this protein is regulated by translational controls in these tissues during hibernation. Previous studies have shown that despite an overall strong suppression of the rate of protein synthesis during hibernation that includes 
disaggregation of most polysomes, the synthesis of selected proteins continues (Hittel and Storey, 2002; Knight et al., 2000; Frerichs et al., 1998). Preferential translation of a specific subset of transcripts during hibernation can be regulated in different ways including sequestering selected transcript types with the polysome fraction whereas most other mRNA types are moved into the translationally silent monosome fraction. Such selective distribution was seen previously with the expression of the fatty acid binding protein (FABP) during hibernation (Hittel and Storey, 2001) and may also apply to the translation of $b c l-x l$. Hence, even with lower mRNA levels, translation of $b c l-x l$ in BAT and skeletal muscle could continue during hibernation resulting in elevated Bcl-XL protein.

A pro-oxidative and pro-apoptotic environment can occur in hibernator organs, especially during arousal from torpor, and in particular in the two tissues (BAT and skeletal muscle) that are involved in thermogenesis. Indeed, Barja de Quiroga (1992) reported that activated brown fat experiences oxidative stress due to the rapid increase in oxygen consumption (and associated increase reactive oxygen species generation) occurring during thermogenesis. Not surprisingly, then, BAT and other hibernator organs show strong anti-oxidant defenses (Buzadzic, et al., 1990; Carey et al., 2000; Eddy et al., 2005b) and would also require enhanced anti-apoptosis mechanisms to deal effectively with the potential for oxidative stress mediated stimulation of apoptosis during arousal from hibernation. Whereas Bcl-XL could provide this action in four of the organs tested in this study (BAT, heart, kidney, muscle), the lack of change in $b c l$-xl transcript or Bcl$\mathrm{XL}$ protein levels in brain and liver of hibernating ground squirrels suggested the possibility that another member of the Bcl-2 protein family might also contribute to anti- 
apoptotic defense during hibernation..

Quantification of Bcl-2 protein levels in ground squirrel tissues showed that this was true. Bcl-2 protein content was greatly increased in brain (by 4.7 -fold) and 2-3 fold increases were also seen in heart, kidney and liver of hibernating squirrels (Fig. 5.6). However, BAT showed unchanged levels of $\mathrm{Bcl}-2$ and $\mathrm{Bcl}-2$ decreased by $48 \%$ in skeletal muscle during hibernation. Hence, it is apparent that elevated levels of one or both of $\mathrm{Bcl}-\mathrm{XL}$ or $\mathrm{Bcl}-2$ provide the organs of torpid ground squirrels with anti-apoptotic defense.

$\mathrm{BAD}$ is a pro-apoptotic protein that counteracts the survival function of Bcl-XL and Bcl-2. To reduce pro-apoptotic stress during hibernation, I hypothesized that BAD should be reduced or inactivated. Since heart and skeletal muscle showed distinctly different patterns of response by anti-apoptotic protein $(\mathrm{Bcl}-2)$, I reasoned that these two organs should illustrate the range of responses by the pro-apoptotic BAD during hibernation. The data show that the overall content of $\mathrm{BAD}$ was unchanged in these organs during hibernation but the phosphorylation state of the protein was markedly altered. In heart, the phosphorylation of all three sites (Ser 112, Ser 136, Ser 155) increased strongly during hibernation (the content of phosphoenzyme rose by 1.92-, 1.29and 2.66-fold, respectively) (Fig. 5.8). Phosphorylation is known to inhibit BAD by two mechanisms (Tan et al., 2000); phosphorylation at both Ser 112 and 136 leads to binding to 14-3-3 proteins to sequester BAD in the cytosol whereas phosphorylation at Ser 155 blocks the binding to Bcl-XL. Hence, during hibernation in heart a key pro-apoptotic marker (BAD) is suppressed where as key anti-apoptotic proteins (Bcl-XL, Bcl-2) are enhanced. This indicates an overall balance in favor of anti-apoptotic signaling in the 
heart of the thirteen-lined ground squirrels.

By contrast, hibernation had very different effects on the phosphorylation of BAD in skeletal muscle; the content of phospho-BAD Ser 112 was unaltered but phospho-BAD Ser 136 and phospho-BAD Ser 155 contents where strongly reduced by $46 \%$ and $52 \%$ during hibernation (Fig. 5.8). Hence, pro-apoptotic signaling could be enhanced in skeletal muscle during hibernation although this might be balanced by the elevated $\mathrm{Bcl}$ XL content. Alternatively, other anti-apoptotic signaling pathways and mechanisms such as those involving inhibitors of apoptosis, cIAP and XIAP, might be active during hibernation to promote muscle cells survival (Vaux and Silke, 2005).

Analysis of the amino acid sequence of Bcl-XL from thirteen-lined ground squirrels revealed a strong homology (95-98\%) with the protein from nonhibernating animals (human, mouse, rat) (Fig. 5.2 and 5.3). Several amino acid substitutions occurred in the ground squirrel sequence including substitution of a glutamine residue substitution for a histidine residue at position 58 and a substitution of isoleucine for a valine at position 65 (Fig. 5.2). All of these substitutions are replacements with a similar amino acid (very close to the same hydrophobicity). Nonetheless, these substitutions could provide minor alterations to protein conformation that benefit protein function at the low body temperature during hibernation. Several cases of unique amino acid substitutions to the sequences of hibernator proteins have been previously reported. This includes substitutions in GRP78 (Chapter 2) and selected substitutions in the FABP sequence in both ground squirrels and bats which affects key functional groups and appear to aid substrate binding at low temperature (Hittel and Storey, 2001; Eddy and Storey, 2004; Morin and Storey, 2005). 
In conclusion, I present evidence that potent anti-apoptotic proteins in the $\mathrm{Bcl}-2$ family of proteins, $\mathrm{Bcl}-\mathrm{XL}$ and $\mathrm{Bcl}-2$, respond positively to hibernation with increased expression in selected tissues of thirteen-lined ground squirrel S. tridecemlineatus. In addition, the pro-apoptotic protein, BAD, is effectively inactivated in heart during hibernation by strongly enhanced phosphorylation at all three phospho-serine sites. These results suggest that cellular mechanisms of apoptosis were suppressed during hibernation. Furthermore, it has been shown that Bcl-XL and $\mathrm{Bcl}-2$ are also involved in the regulation of cell cycle arrest (Janumyan et al., 2003). It will be interesting in future studies to investigate whether a similar pattern of anti-apoptotic protein expression is also seen during hibernation when different mechanisms of anti-apoptosis are evaluated, such as those involving inhibitors of apoptosis (XIAP and cIAP). 
Fig. 5.1: Partially amplified $b c l-x l$ cDNA sequence and deduced amino acid sequence of Bcl-XL from brain of thirteen-lined ground squirrels. Nucleotides and amino acids are numbered on the left. A single open reading frame was predicted from the nucleotide sequence and coded for a polypeptide with 146 residues. Note that the full length protein is 233 amino acids for mammalian species including human, mouse and rat. 


\section{Fig. 5.1}

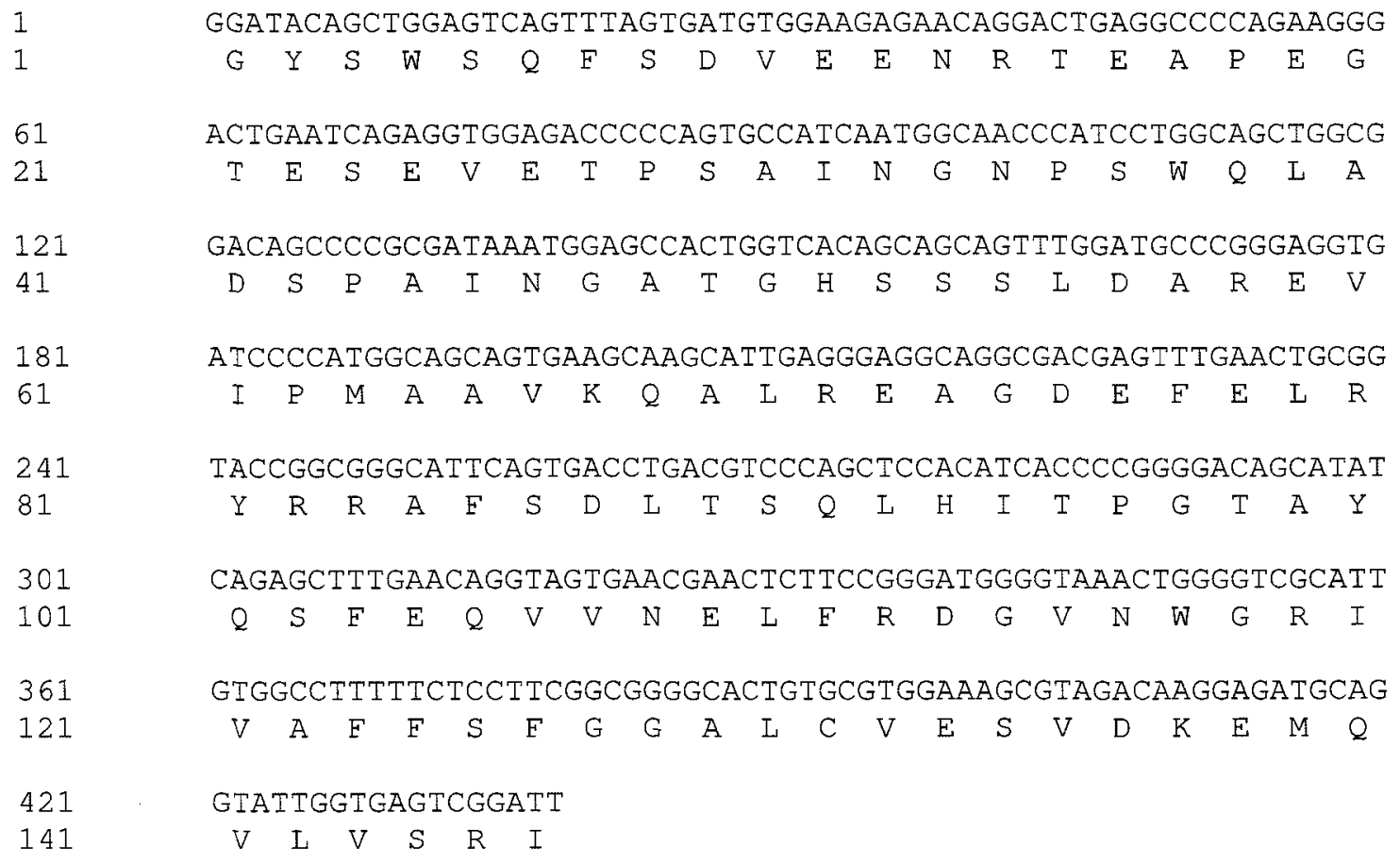


Fig. 5.2: Partial amino acid sequence of Bcl-XL from thirteen-lined ground squirrel ( $S$. tridecemlineatus) aligned with human (Homo sapiens), mouse (Mus musculus) and rat (Rattus norvegicus) Bcl-XL amino acid sequences (Genbank accession numbers DQ503423, BT007208, X83574, and U34963, respectively). Distinct amino acid substitutions in the squirrel sequence are indicated in bold underline. Dashes (-) represent amino acids in the human, mouse and rat sequences that are identical with the squirrel sequence; spacer dots are inserted when residues are not present in all sequences. 


\section{Fig. 5.2}

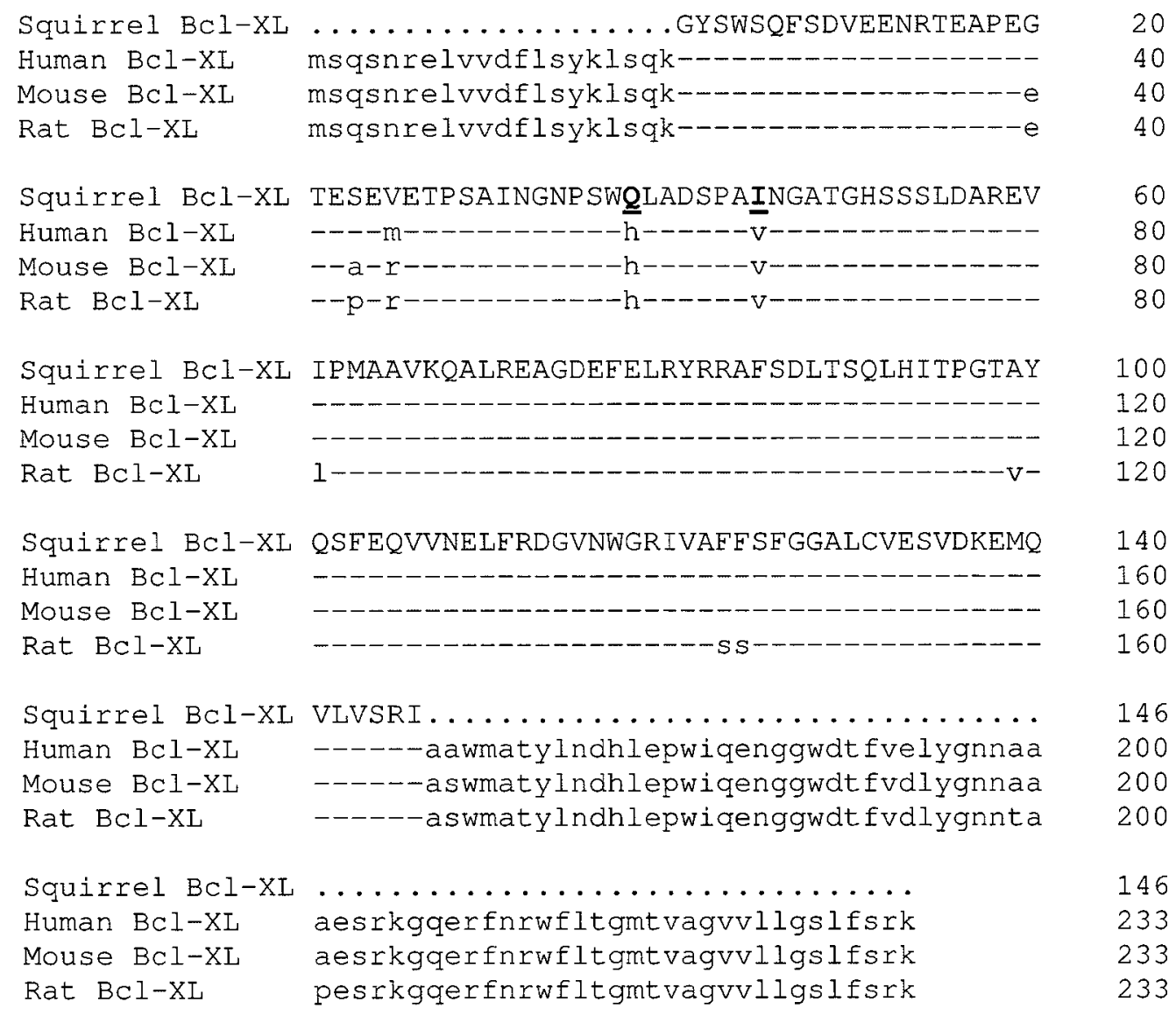


Fig. 5.3: (A) Homology tree prepared with DNAMAN showing the percent identities between the nucleotide sequences of $b c l-x l$ from ground squirrel (S. tridecemlineatus), human, mouse and rat (accession numbers as in Fig.5.2). (B) Cladogram depicting amino acid identity of the thirteen-lined ground squirrel Bcl-XL partial sequence (146 residues) compared with Bcl-XL protein of other mammalian species: human, mouse and rat. 
Fig. 5.3

\section{A. GENE}
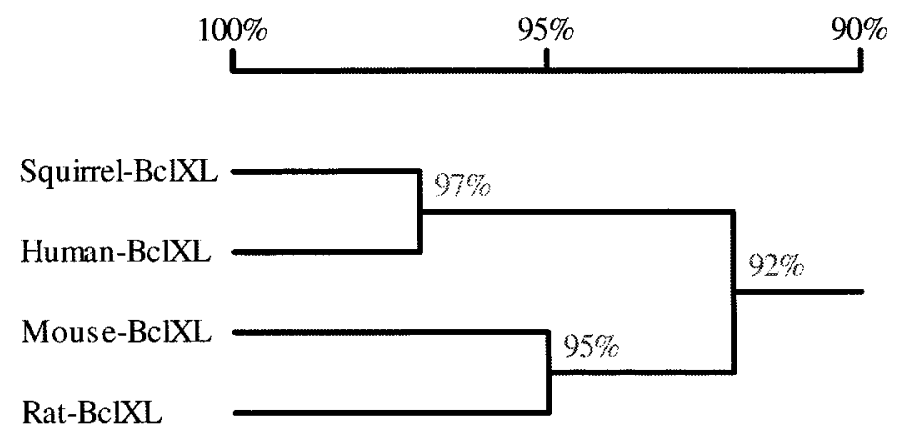

B. PROTEIN

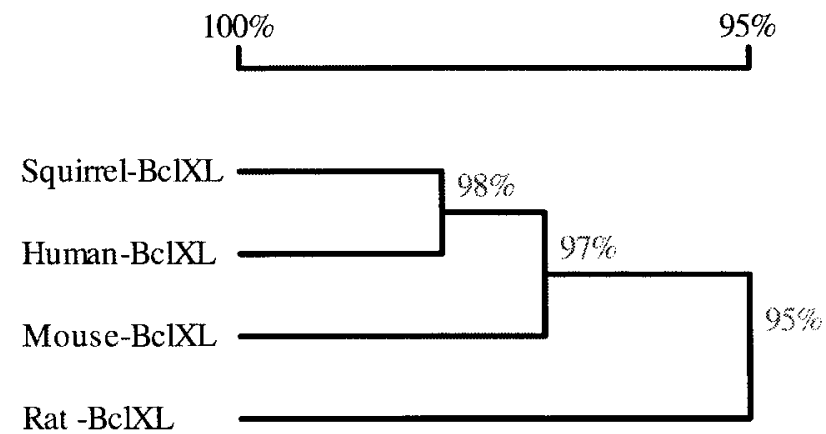


Fig. 5.4: Analysis of $b c l-x l$ transcript levels in ground squirrel tissues during hibernation. (A) Representative RT-PCR of $b c l-x l$, relative to alpha-tubulin in euthermic (E) and hibernating $(\mathrm{H})$ ground squirrel tissues. (B) Bar graphs representing $b c l$ - $x l$ mRNA levels in the organs of ground squirrels. The lowest dilutions for alpha-tubulin and $b c l-x l$ which have visible PCR products (normally at dilution $10^{-3} \sim 10^{-4}$ ) were chosen for quantification. Statistical testing of normalized band intensities from euthermic versus hibernating samples used the Student's $t$-test. * and **-Values for hibernating sample are significantly different from the corresponding euthermic values, $\mathrm{P}<0.05$ and $\mathrm{P}<0.01$ respectively. Data are mean ratios \pm S.E.M., $n=3$ independent trials with both hibernating and euthermic samples. Error bars shown on the histograms are the S.E.M calculated on the ratios of hibernating to euthermic values. 
Fig. 5.4

A.

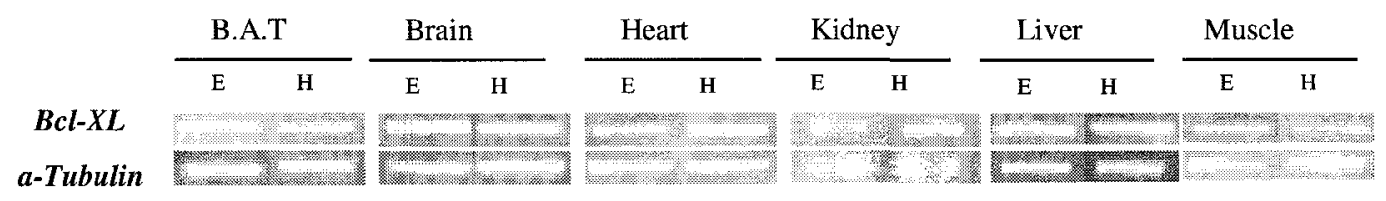

B.

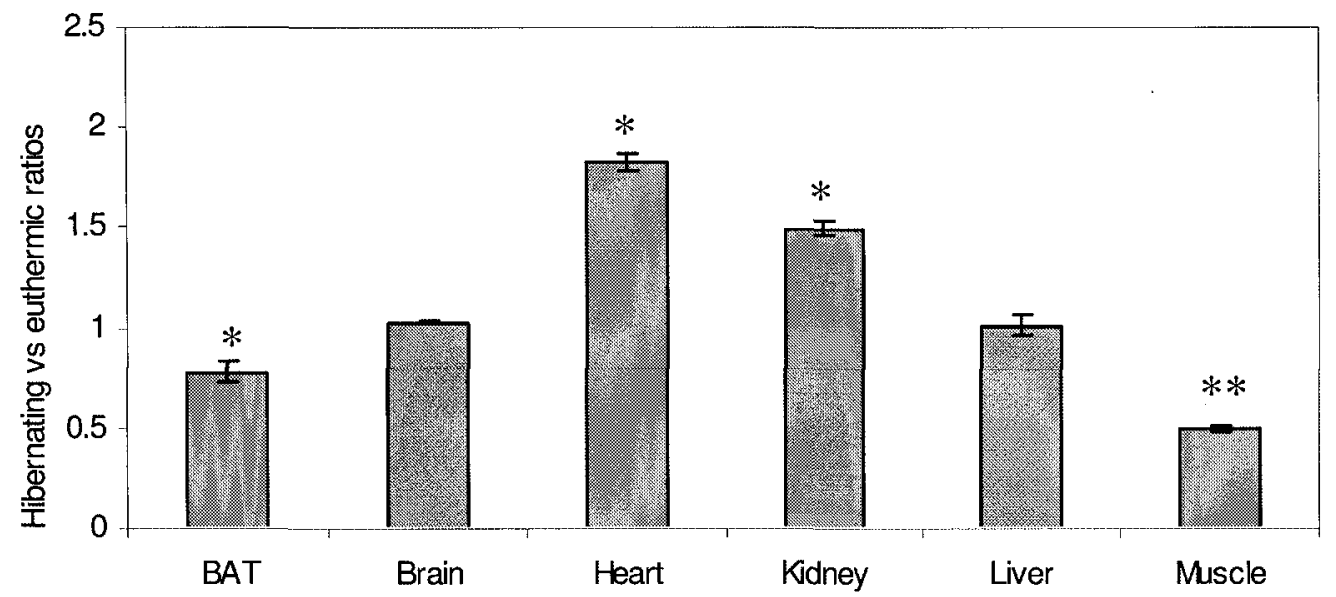


Fig. 5.5: Bcl-XL protein expression in six tissues of thirteen-lined ground squirrels. (A) Representative Western blots showing Bcl-XL protein band at $\sim 28 \mathrm{kDa}$ in tissues of euthermic (E) and hibernating $(\mathrm{H})$ ground squirrels. (B) Histograms showing the relative Bcl-XL protein levels in ground squirrels organs. Data are mean ratios \pm S.E.M., $n=3$ independent trials. * - Values for hibernating samples are significantly different from the corresponding euthermic values using the two-tailed Student's $t$-test, $\mathrm{P}<0.05$; ** $-\mathrm{P}<$ $0.01, \mathrm{n}=3$ trials. 
Fig. 5.5

A.

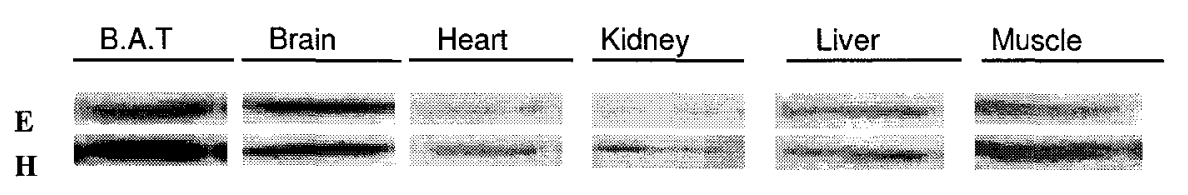

B.

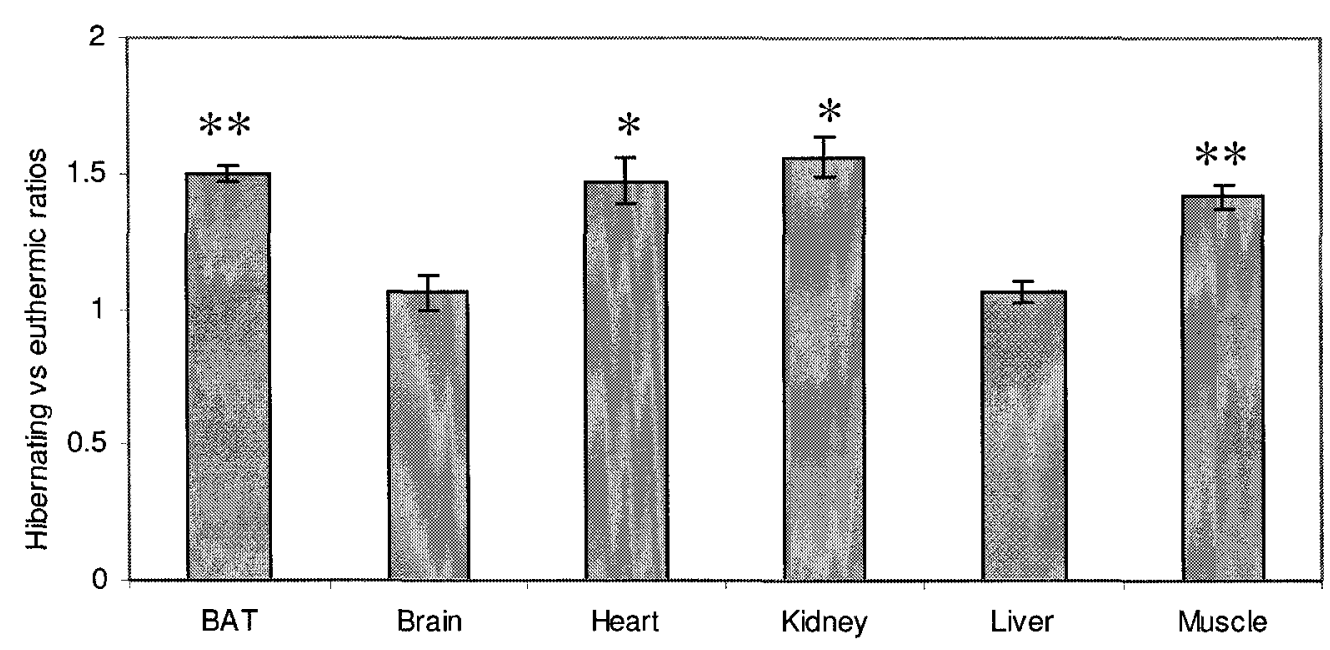


Fig. 5.6: $\mathrm{Bcl}-2$ protein expression in six tissues of thirteen-lined ground squirrels. (A) Representative Western blots showing $\mathrm{Bcl}-2$ protein band at $\sim 26 \mathrm{kDa}$ in tissues of euthermic (E) and hibernating $(\mathrm{H})$ ground squirrels. (B) Histograms showing the relative Bcl-2 protein levels in ground squirrels organs. Data are mean ratios \pm S.E.M., $n=3$ independent trials. * - Values for hibernating samples are significantly different from the corresponding euthermic values using the two-tailed Student's $t$-test, $\mathrm{P}<0.05$; ** $-\mathrm{P}<$ $0.01, \mathrm{n}=3$ trials. 
Fig. 5.6

A.

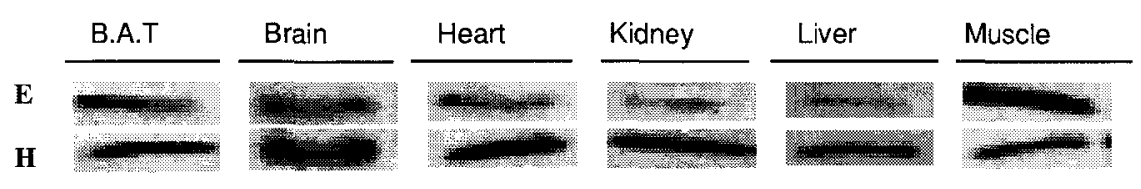

B.

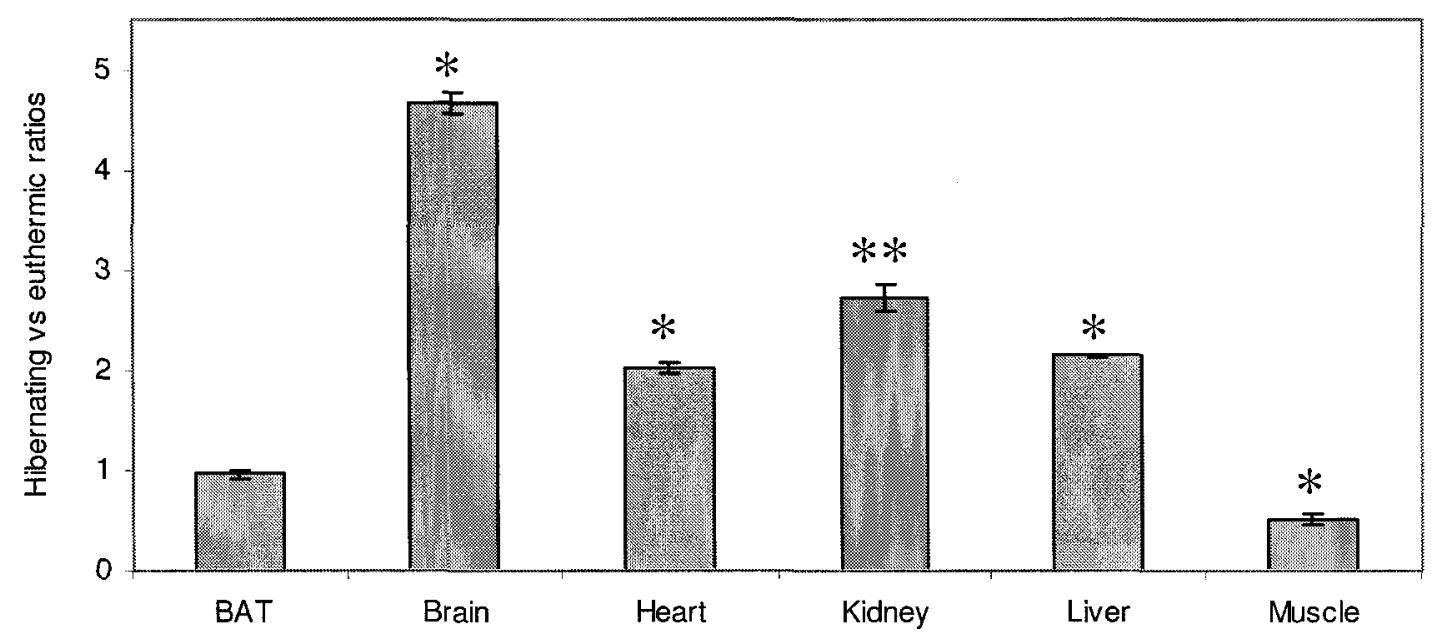


Fig. 5.7: BAD protein expression in heart and muscle of thirteen-lined ground squirrels.

(A) Representative Western blots showing BAD protein band at $\sim 23 \mathrm{kDa}$ in euthermic (E) and hibernating $(\mathrm{H})$ ground squirrels organs. (B) Histograms showing the relative BAD protein levels in ground squirrels organs. Data are mean ratios \pm S.E.M., $n=3$ independent trials. 
Fig. 5.7

A.

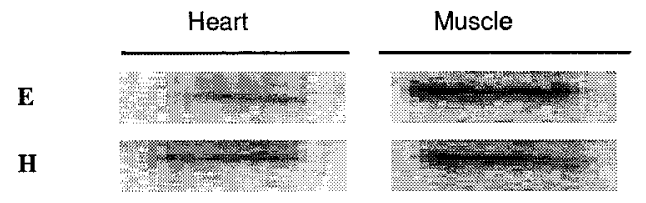

B.

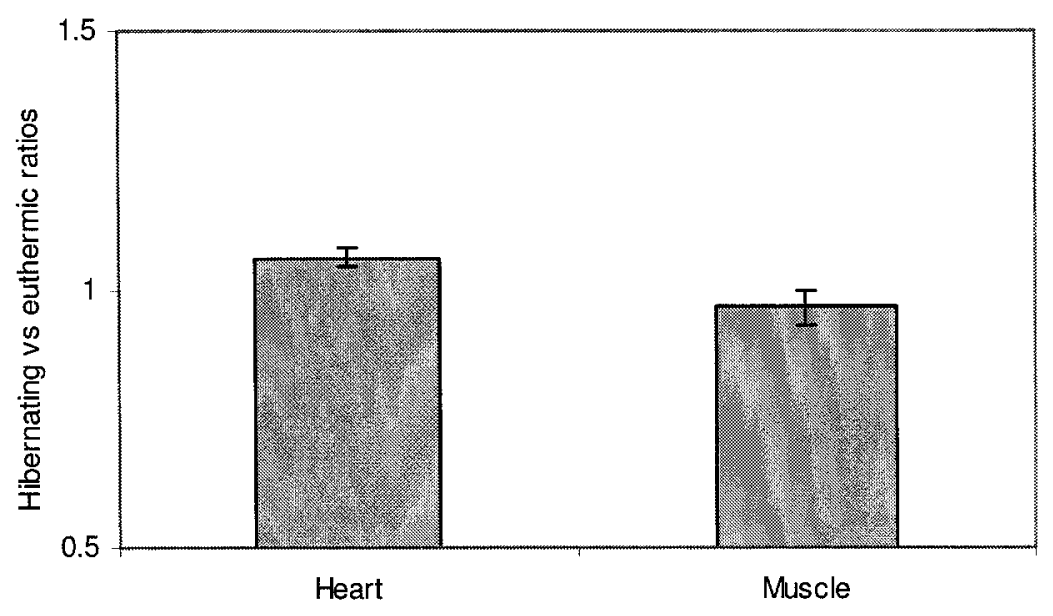


Fig. 5.8: Protein expression of three phosphorylated forms of BAD in heart and muscle of thirteen-lined ground squirrels. (A) Representative Western blots showing pBAD-Ser 112, pBAD-Ser 136 and pBAD-Ser 155 protein bands at $\sim 23 \mathrm{kDa}$ in euthermic $(\mathrm{E})$ and hibernating $(\mathrm{H})$ ground squirrels organs. (B) Histograms showing the mean relative protein levels in ground squirrels organs. Data are means \pm S.E.M., $n=3$ independent trials. *- and ** - Values for hibernating samples are significantly different from the corresponding euthermic values using the two-tailed Student's $t$-test, $\mathrm{P}<0.05$ and $\mathrm{P}<$ 0.01 respectively, $\mathrm{n}=3$ trials. 
Fig. 5.8

A.

\begin{tabular}{|c|c|c|c|c|c|c|c|}
\hline \multicolumn{2}{|c|}{ pBAD Ser 112} & & \multicolumn{2}{|c|}{ pBAD Ser 136} & & \multicolumn{2}{|c|}{ pBAD Ser 155} \\
\hline Heart & Muscle & & Heart & Muscle & & Heart & Muscle \\
\hline $2-2=$ & $2 x=0$ & $\begin{array}{l}E \\
H\end{array}$ & & $=$ & E & 2 & feses \\
\hline
\end{tabular}

B.

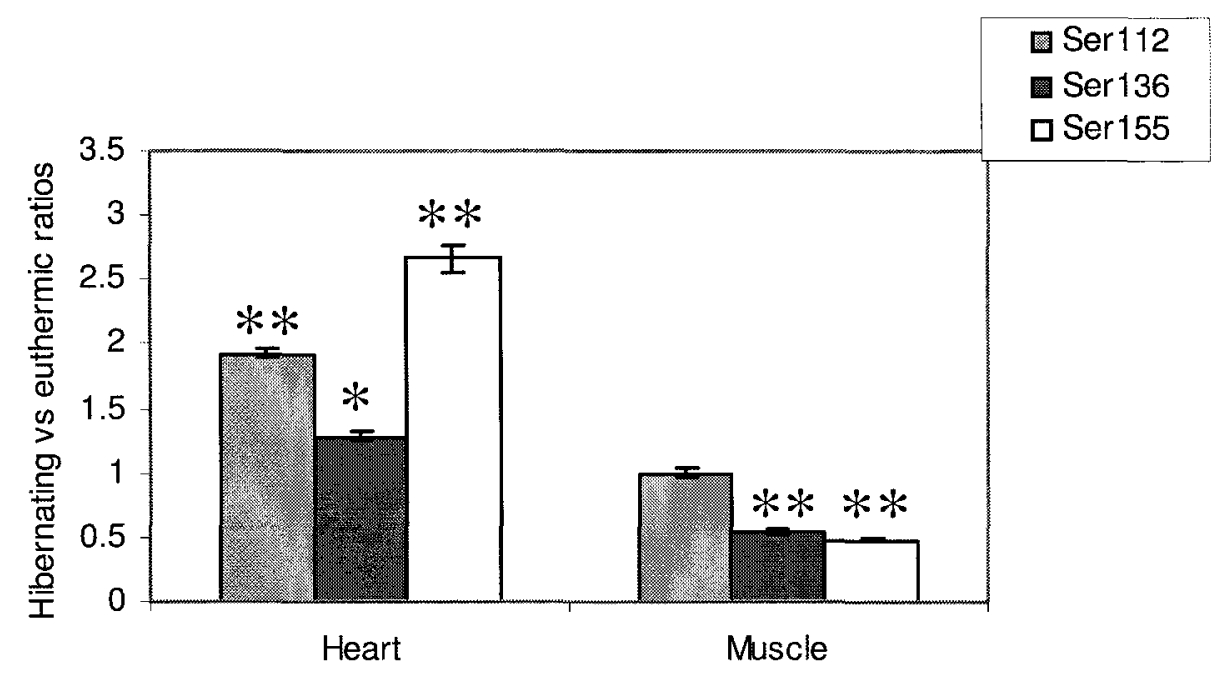




\section{Chapter 6}

\section{General Discussion}


Hibernation is characterized by periods of deep torpor (including an extreme reduction of $\mathrm{Tb}$, heart rate, blood flow and oxygen consumption) that are interrupted by short periods of arousal (body temperature rises back to $\sim 37^{\circ} \mathrm{C}$ and physiological parameters are rapidly restored to near normal levels). In order to coordinate the biochemical and physiological processes that help to conserve energy consumption during torpor, hibernators up-regulate genes that perform crucial functions for the hibernation phenotype and down-regulate other gene products that are not essential during torpor. The work presented in this thesis highlights multiple mechanisms of molecular response to hibernation: changes in gene expression, changes in protein levels, posttranslational control of protein function by reversible phosphorylation, and altered subcellular localization of proteins.

Gene expression can be controlled at both transcriptional and translational levels. A variety of genes are known to be up-regulated during hibernation; and this generally results from the binding of a transcription factor to its response element, at the promoter region of the particular gene, resulting in an increased level of transcription. By contrast, hibernating mammals also decrease the abundance of other gene products via multiple mechanisms. This can be achieved by reducing the rate of gene transcription or via phosphorylation or degradation of the transcription factor. Gene expression can be also regulated at the levels of mRNA processing and stability including mRNA turnover, splicing of pre-mRNA, and 5'decapping of the transcript that precede the export of the mature mRNA from the nucleus into the cytosol (Mata et al., 2005). At the translational level, control can also be applied at the initiation and elongation phase of the protein synthesis as well as by the stability and post-translational modifications of the proteins. 
Through the repeated cycles of torpor and arousal during the hibernation season, mammalian hibernators employ the mechanism of reversible phosphorylation (via the actions of protein kinases and protein phosphatases) to switch metabolic functions between euthermic and hypometabolic states.

Overall, the studies reported in this thesis demonstrate that the differential expression of genes involved in the unfolded protein response, in the inhibition of muscle atrophy, and in anti-apoptotic defense play an important role in ground squirrels hibernation. A variety of techniques, including semi-quantitative RT-PCR and Western blotting, were used to examine the expression of a variety of genes and proteins, assess post-translational modification of selected proteins, and study the subcellular localization of selected transcription factors. In addition, DNA-binding of selected transcription factors to their response element was assessed via the electrophoretic mobility shift assay and TransAM techniques. Taken together, these multiple techniques provided a comprehensive view of how cells regulate gene expression at different levels, and provided insights on the use of transcription factors to coordinate multiple regulatory mechanisms of gene expression to properly exert biological functions required by ground squirrels for hibernation success.

\section{Gene and protein regulation during torpor}

Many aspects of hibernation that are natural for hibernating species are highly stressful for nonhibernating mammals. These include low core Tb (hypothermia), low organ perfusion rates (ischemia), oxidative stress (especially of the magnitude that occurs during arousal), greatly suppressed physiological functions (e.g. kidney filtration, muscle 
inactivity). Most of these conditions are known to trigger endoplasmic reticulum (ER) stress in nonhibernating mammals and activate the unfolded protein response signaling pathways. However, hibernators possess adaptive responses that combat cellular stress and protect vital organs both during torpor and during transitions to/from the hypometabolic state.

In Chapter 2, I examined the expression of a key biomarker of ER stress, the glucose regulated protein $78 \mathrm{kDa}$ (GRP78) in different organs of euthermic and hibernating thirteen-lined ground squirrels. GRP78 expression was shown to increase at both the mRNA and protein level in BAT and brain of hibernating ground squirrels (Fig. 2.2 and Fig. 2.7), two highly oxygen-dependent organs that are critical to whole animal survival during winter. It is well known that GRP78 is involved in the folding and assembly of newly synthesized proteins. Increased levels of GRP78 in these key organs BAT that must provide heating both during torpor and for arousal, and brain that must maintain its sensing and signaling capacity throughout torpor - suggests a pivotal role for GRP78 in folding hibernating-responsive proteins, maintaining cellular homeostasis, and balancing cell survival versus apoptosis during hibernation. Additional studies that I have undertaken but are not reported in this thesis include the analysis of GRP78 protein and transcript responses in BAT and brain of a different type of hibernator, the little brown bat Myotis lucifugus. The data showed unchanged levels of the protein between euthermic and hibernating animals and up-regulation of $\operatorname{grp} 78 \mathrm{mRNA}$ levels during hibernation (Fig. 6.1). Hence, at the level of mRNA expression, the responses by the two hibernating species concur whereas the data on protein levels provides a complement to the study of Lee et al. (2002) who reported increased amounts of GRP78 protein in brain of bats 
(Rhinolopus ferrumequinum), within the first 30 min of arousal, compared with levels in the torpid state. Hence, it is highly likely that the response seen in $M$. lucifugus represents an "anticipatory" up-regulation of $g r p 78$ transcripts in order to support a rapid synthesis of the GRP78 protein during arousal, a time when the key chaperone function of the protein would be very important. Thus, enhanced expression of GRP78, a key component of the UPR response to cellular stress appears to be an integral response in hibernating species to stresses encountered in torpor-arousal cycles although the timing of protein expression differs somewhat between rodent and bat species.

Given the fact that GRP78 is an integral part of the UPR and that $g r p 78$ transcript levels were up-regulated in BAT and brain of thirteen-lined ground squirrels (by 3.5- and 4.1-fold respectively), evidence was provided in Chapter 2 that these two organs experienced ER stress (perturbation of normal physiological state of the ER) during hibernation. I then hypothesized that the UPR would be activated during torpor to provide proactive protection to cells to preserve and stabilize cellular proteins and the protein synthesizing machinery over long periods of cold torpor. Chapter 3 examined the expression of ATF4, a central regulator of the UPR, at both the mRNA and protein levels in BAT, brain and other organs of $S$. tridecemlineatus in order to evaluate its role in hibernation. The responses of a variety of proteins involved in the UPR were also evaluated. The data indicated that ATF4 was elevated in both BAT and brain by 2.51- and 1.88-fold respectively during hibernation (Fig. 3.3B). Although strong suppression of the overall rate of protein synthesis occurs during hibernation, the synthesis of selected proteins continues (Hittel and Storey, 2002; Knight et al., 2000; Frerichs et al., 1998); this included translation of grp 78 and activating transcription factor 4 (atf 4 ) genes with 
elevated levels of the corresponding proteins in BAT and brain of hibernating squirrels. Another level of post-transcriptional control takes place during initiation of translation. Globally, translation of most transcripts is regulated by changes in the phosphorylation state of translation initiation factors; as reported by Kozak (2002), phosphorylation of eIF2 $\alpha$ activates translation of mRNAs encoding several short upstream open reading frames (uORFs) containing multiple start codons (AUGs.) at the 5'-end of the transcript. In addition, it has been reported that translation of a distinct group of mRNAs is mediated through control elements present in the UTRs of target transcripts (Mata et al., 2005). All these features in the 5 'end of the mRNA allow translation of the corresponding genes without translational regulation by eIF2 $\alpha$ which inhibits translation when phosphorylated. One of these genes that is known to escape translational attenuation by eIF2 $\alpha$ is atf 4 ; its translation is mediated instead through an internal ribosomal entry site (IRES) located in the 5'UTR of the atf4 mRNA (Fernandez et al., 2002). My data also shows an increased level of ATF4 despite the inhibition of over all protein synthesis during hibernation.

The positive correlation between the transcript levels of grp78 and ATF4 protein levels led to the investigation of ATF4 DNA-binding to the ATF/CRE-like site in the promoter region of $g r p 78$. It has been previously reported that ATF4 activates grp78 gene in response to various conditions that trigger ER stress (Luo et al., 2003). I examined whether ATF4 could also regulate $g r p 78$ in brain of ground squirrels, a tissue that showed increased levels of ATF4 protein and the biggest increase in grp 78 transcript levels during hibernation. My data in Chapter 3 demonstrated that ATF4 is more transcriptionally active during torpor than euthermia (Fig. 2.6). Hence, ATF4 could be the transcription 
factor responsible for $\operatorname{grp} 78$ up-regulation during hibernation.

Reversible phosphorylation of proteins is the most common post-translational modification used by hibernators to activate or inactivate proteins in order to regulate hibernation at the molecular level. To examine the relevance of this mechanism to the regulation of the UPR in hibernation, I analyzed the phosphorylation status of CREB-1 (cAMP response element binding protein 1), a nuclear co-factor that forms a complex with ATF4 and binds to the promoter region target genes. My data indicated that hibernation triggers the induction of CREB1 phosphorylation in all of the tissues of thirteen-lined ground squirrels that were studied. Hence, p-CREB1 that translocated into the nucleus during hibernation would strongly increase the amount of ATF4•p-CREB1 complex present and thereby increase ATF/CRE binding and the activation of target genes under their control including the pro-survival gene grp78 (Luo et al., 2003). This is consistent with the findings of MacDonald and Storey (2005) who reported high levels of phospho-CREB1 and other highly phosphorylated proteins including p38 MAPK, ATF-2 and Hsp27 during hibernation in selected organs of Richardson's ground squirrels ( $S$. richardsonii). Phosphorylation of the above proteins, in this case, led to the activation of their downstream kinase or transcriptional function which elicits adaptive responses in organs during hibernation. However, examples of protein inactivation through phosphorylation have also been reported during hibernation. In Chapter 4, high levels of phospho-FoxO1a were observed in heart and skeletal muscle of hibernating ground squirrels, as well as decreased FoxO1a DNA-binding and nuclear exclusion of the phosphorylated form of FoxO1a that is inactive (Fig. 4.2, Fig. 4.3, and Fig. 4.4). Recently, Van Der Heide (2004) has reported that proteins of the FoxO family are 
regulated at three levels: transcriptional activity (including modulation of DNA binding, acetylation/de-acetylation and association with co-factors), localization (shuttling between nucleus and cytosol), and stability (proteolysis and cleavage). Data provided in Chapter 4 support these levels of FoxO regulation. The degradation of FoxO proteins adds an additional layer of negative regulation of protein activity that relies on the shuttling system. Whether degradation of FoxOla occurred following its relocation into the cytosol has not yet been investigated in organs of hibernating ground squirrels. Further studies on FoxO1a processing by the ubiquitin-proteasome system or by other proteases are warranted to better define the stability of the protein during hibernation, since it may be disadvantageous to rely only on dephosphorylation and translocation of FoxO1a to suppress its gene regulating activity during torpor.

\section{Unfolded protein response}

It is well known that the UPR can be activated by several conditions including amino acid deprivation, hypoxia and oxidative stress. Such conditions trigger ER stress and the cells respond by activating the UPR (Fig. 4.1) resulting in the induction of molecular chaperones, upregulation of the ERAD (ER Associated Degradation) and phospholipids synthesis, and induction of apoptosis (Schröder and Kaufman, 2005). In ground squirrel tissues, ER stress may arise particularly from oxidative stress during arousal. Indeed, a variety of studies have shown that hibernators have enhanced antioxidant defenses including increased levels of antioxidant metabolites (e.g. ascorbate), elevated activities of antioxidant enzymes, and hibernation responsive upregulation of various antioxidant genes (Buzadzic et al., 1990; Drew et al., 2002; Storey, 
2003; Eddy et al., 2005b). I hypothesized that the UPR would be activated during torpor to provide proactive protection to cells to preserve and stabilize cellular proteins and the protein synthesizing machinery over long periods of cold torpor. I assessed the protein levels of several genes that are part of the main pathway of the UPR, the PERK/eIF2 $\alpha /$ ATF4 pathway which regulates the expression of both pro-survival and proapoptotic genes. In addition to elevated levels of ATF4 and GRP78 protein in BAT, brain and skeletal muscle during hibernation, my studies in Chapter 3 demonstrated that expression of pPERK, one of the eIF2 $\alpha$ kinases was unchanged. This occurred despite the elevated level of phosphorylated eIF2 $\alpha$ in selected organs of ground squirrel as reported by Frerichs et al. (1998) and Hittel and Storey (2002). Hence, another kinase such as GCN2, PKR and HRI (Clemens, 2001) may mediate eIF2 $\alpha$ phosphorylation during hibernation. The reduced level of GADD34 was also consistent and in line with the suggestion of strongly suppressed protein synthesis during torpor. Reduced levels of GADD34 would help to keep eIF2 $\alpha$ in the phosphorylated inactive state during torpor. I also examined expression of ATF6 $50 \mathrm{kDa}$, implicated in another signaling pathway of the UPR. ATF6 $50 \mathrm{kDa}$ protein levels did not change between euthermic and hibernating ground squirrels heart; however, its translocation into the nucleus during hibernation requires further investigation in order to elucidate the changes that occur through the ATF6 pathway in heart of hibernating squirrels. ATF4 involvement in ER stress links both branches of the UPR (the PERK/eIF2 $\alpha /$ ATF4 pathway and the ATF6 pathway) with the induction of the pro-apoptotic CHOP protein and pro-survival GRP78 protein. The finding that CHOP was elevated in the heart during hibernation whereas decreased or unchanged amounts were found in other tissues warrants further investigation to evaluate 
organ specific requirements during torpor. It is known that CHOP is robustly expressed following disruption of homeostasis in the ER but its role in the stress response has remained largely undefined. However, recent studies revealed that elevated CHOP expression results in the down-regulation of expression of $\mathrm{Bcl}-2$, an anti-apoptotic gene (McCullough et al., 2001). As shown in Chapter 5, Bcl-2 protein levels increased in several tissues of ground squirrel during hibernation, including heart (Fig. 5.6). Hence, it would be interesting to investigate the various roles that $\mathrm{CHOP}$ plays during hibernation in different organs of ground squirrels.

The data gathered here reinforces the importance of transcription factors in the regulation of hibernation-responsive genes and the differential expression of proteins that maintain the hibernation phenotype. These studies suggest an important integrated response between ER signaling and cellular adaptation during torpor and provides initial evidence that ER stress and the UPR may be a key component of hibernation in thirteenlined ground squirrels.

\section{Muscle disuse atrophy}

Hibernators use the activation or inactivation of selected transcription factors to alter or regulate metabolic functions that address specific problems in torpor survival. Given the evidence that hibernating mammals experience a dramatic reduction in locomotor activity during torpor and that cardiac muscle in hibernators must function at both a lower rate and a low $\mathrm{Tb}$, I hypothesized that selected genes involved in muscle disuse atrophy may be down-regulated and their protein products inactivated during torpor to minimize atrophy and defend muscle functions. It has been reported recently that skeletal muscle 
disuse atrophy results from both a decrease in protein synthesis and an increase in protein degradation (Jackman and Kandarian, 2004). Potential triggers and signaling molecules involved in muscle disuse atrophy have just begun to be identified (Fig. 6.2). In Chapter 4, I examined FoxOla transcript and protein levels, as well as amounts of phosphoFoxOla Ser 256, and the levels of selected proteins associated with muscle atrophy in heart and skeletal muscle. FoxOla transcript and protein increased significantly in heart during torpor, but were unchanged in muscle (Fig. 4.1). However, phospho-FoxO1a Ser 256 content increased in both tissues. Thus, even though heart and muscle showed different patterns of foxOla gene and protein expression, the data suggest that FoxOla is inactivated in both tissues during torpor. Consistent with this, FoxOla DNA-binding activity did not change in heart and decreased in muscle; in parallel with this, levels of MAFbx (atrogin-1), a downstream gene regulated by FoxO1a, showed no significant changes in heart but decreased slightly in muscle during torpor (Fig. 4.5). By contrast, Bcl-3 protein levels were elevated in both tissues whereas NFkB p50 protein increased in heart and showed no change in muscle during torpor (Fig. 4.6 and Fig. 4.7). Moreover, the distribution of $\mathrm{NFKB}$ p50 in subcellular fractions of heart and muscle showed increased levels of $\mathrm{Nf} \kappa \mathrm{B}$ p50 in cytoplasmic extracts of both tissues during hibernation as compared with euthermia (Fig. 4.8B), indicating that this transcription factor was retained in the cytosol where its transcriptional activity would be curtailed. Similar results were observed for Bcl-3 in the cytoplasmic fraction from heart (the only organ tested) of hibernating squirrels; this showed high levels of Bcl-3 in the cytoplasmic fraction of torpid animals (Fig. 4.9). Thus, although previous studies on ground squirrels ( $S$. lateralis) demonstrated a slight atrophy of skeletal muscle during torpor, the overall 
results presented in this thesis shows down-regulation or inactivation of selected atrophyrelated genes during hibernation. Even though skeletal muscle of $S$. lateralis showed increased levels of MAFbx mRNA, the protein content in skeletal muscle of $S$. tridecemlineatus decreased during hibernation. This suggests that ground squirrel muscle undergoes little or no atrophy during torpor. FoxO protein expression has also been linked to decreased muscle fibre size via MAFbx whereas NFKB signaling seems to be associated instead with protein loss (reduction in muscle fibre protein content) (Sandri et al., 2004). It will be interesting to address future studies on a new signaling pathway involving $\mathrm{NFKB}$ and a downstream target MuRF1 which induces both a decrease in muscle fibre size and protein breakdown via the up-regulation of the E3 ubiquitin-ligase MuRF1.

\section{Anti-apoptotic defense during torpor}

During the hibernating period, ground squirrel organs are exposed to a prooxidative and pro-apoptotic environment, resulting from the generation of ROS particularly during arousal from torpor when blood flow, oxygen delivery and oxygen consumption increase rapidly. It is well-established that hibernators show adaptations of their antioxidant defenses over the winter season and the data in the present thesis show that anti-apoptotic defense is also a component of the hibernation phenotype. In Chapter 5, I studied the intrinsic pathway of apoptosis involving proteins of the Bcl-2 family that may have anti-apoptotic (Bcl-XL and $\mathrm{Bcl}-2$ ) versus pro-apoptotic (BAD) functions. Gene transcript and protein levels of Bcl-XL (Fig. 5.4 and 5.5), and protein levels of Bcl-2 (Fig. 5.6) and BAD (Fig. 5.7) were quantified, as well as three forms of phosphorylated 
BAD (Fig. 5.8). The data showed organ-specific responses during hibernation. Data from heart showed enhanced expression of $\mathrm{Bcl}-\mathrm{XL}$ and $\mathrm{Bcl}-2$ proteins in hibernating ground squirrels, unchanged levels of BAD and increased amounts of all three phosphorylated forms of BAD. This suggests that anti-apopotosis signals dominate during torpor to promote cell survival in heart during hibernation. Furthermore, it has been reported that the ratio between the anti-apoptotic proteins (antagonists) and the pro-apoptotic proteins (agonists) determines the susceptibility of a cell to a death signal (Gross et al. 1999; Zha et al., 1996) and clearly, the ratio in ground squirrel heart during torpor would strongly favour anti-apoptosis. Skeletal muscle showed a somewhat different response. Protein levels of $\mathrm{Bcl}-\mathrm{XL}$ increased in skeletal muscle whereas levels of Bcl-2, BAD and phospho-BAD Ser 112 remained unchanged during hibernation. Interestingly, protein levels of Bcl-2 protein, as well as Ser 136 and Ser 155 phospho-BAD content, were strongly reduced during torpor. These results suggest that other mechanisms of antiapoptotic signaling may be involved in hibernator skeletal muscle, such as those involving inhibitors of apoptosis (e.g. cIAP, XIAP).

\section{Outlook}

Although this thesis answers a lot of questions about the mechanisms involved in hibernation - signal transduction, the UPR, anti-apoptosis, anti-atrophy - like any scientific study, the answers revealed here lead to more questions. In the early days of hibernation research, the prevailing thought was that the decrease in $\mathrm{Tb}$ and the acidic shift in cell $\mathrm{pH}$ (due to apnoic breathing patterns) were the cause of torpor (pros and cons discussed by Gieser, 1988). However, careful measurements of the patterns of metabolic 
rate reduction and the fall in $\mathrm{Tb}$ proved that the opposite was true - metabolic suppression caused the fall in $\mathrm{Tb}$. Researchers then began to look at the regulatory mechanisms of metabolic suppression as well as other metabolic adjustments needed for hibernation (e.g. adaptations needed to support a long term dependence on lipids as the preferred fuel). These studies in recent years have shown that hibernation is actually a highly complex phenomenon involving regulatory controls over a huge number of cellular functions (including those examined in this thesis) and featuring differential controls applied both within cells and between organs to achieve an overall suppression of metabolic rate, a reorganization of metabolic priorities for optimal long term survival, and noninjurious transitions to/from the hypometabolic state.

Based on the data collected in this thesis, a variety of directions for future study can be suggested. Transcription factor regulation is obviously key to regulating the expression of groups of proteins involved in related cell functions and important strides could be made in hibernation research by screening for changes in gene expression in euthermic versus hibernating states using cDNA microarrays and in transcription factor binding activity using TranSignal protein/DNA arrays (Panomics). In combination, this approach will provide (a) identities of hibernation-responsive genes, including those coding for transcription factors, (b) classification of genes into known groups that are typically under the control of selected transcription factors, and (c) evaluation of the transcriptional activity of selected transcription factors. Since all the transcription factors detected on the Panomics array are well known, the complementary data gathered from cDNA screening will allow links to be made between transcription factors and known downstream genes to suggest new avenues of research into metabolic adjustments that 
define the hibernation phenotype.

The amino acids sequences for the full length ground squirrel GRP78 protein and partial ATF4 and Bcl-XL proteins retrieved in these studies showed 80-99\% identity with the comparable proteins from nonhibernators. Whereas some of the differences may just reflect species-specific substitutions, others appear to be unique to the hibernator. The same is true of a variety of other hibernator proteins that have been sequenced including FoxO1a (Cai et al., 2004a) and fatty acid binding protein (Hittel and Storey, 2001). These unique substitutions may be important in helping to maintain an active protein/enzyme conformation over the wide range of $\mathrm{Tb}$ experienced by hibernators over torpor-arousal cycles. For transcription factors, such substitutions could alter DNA binding in the cold torpid state, perhaps differentially increasing or decreasing the transcriptional activity of these in the hypometabolic state. Hence, important aspects of further work on transcription factors could include determining the full length sequences of selected transcription factors, searching for modifications in key regulatory elements, and testing relative DNA binding at both high and low temperatures.

This thesis addressed four hypotheses about the molecular mechanisms that alter physiological parameters of organs of hibernating ground squirrels so that they can endure extended periods under conditions of reduced metabolic rate, low blood flow, and low body temperatures during torpor, conditions that would be highly injurious or even lethal to most mammals. The study on differential expression of GRP78 was addressed first. The hypothesis stated that hibernators would need to have mechanisms to enhance the production of molecular chaperones such as GRP78, to minimize the misfolding of proteins during synthesis under the unusual cellular conditions of the torpid state. The 
data gathered showed increased levels of GRP78 at both gene and protein levels. This induction of GRP78, raised the possibility that ER stress occurred in selected organs during hibernation and that grp78 was, in part, regulated via the PERK/eIF2 $\alpha / A T F 4$ pathway in response to low temperature, oxidative stress and ischemia/reperfusion effects during hibernation. The second hypothesis stated that ground squirrels would need to modify their UPR response in order to deal with a potential disruption of ER function that could arise under the physiological conditions that accompany hibernation. A recent study on ER stress and oxidative stress has linked ATF4 and Nrf2 signaling pathways, suggesting that phosphorylated form of Nrf2 might also participate in ER stress signaling by promoting the expression of genes that encode antioxidants, or are involved in cell growth, protein folding and cell survival (Cullinan and Diehl, 2006). Hence, another important future direction will be assess the interactions of ATF4 and Nrf2 over the full torpor/arousal cycle in order to gain a complete understanding of the UPR and the ERstress regulated gene as it applies under hibernation conditions. A complementary study can investigate the branch involving the IRE1/ATF6/XBP-1 signaling pathway to examine the degradation state of proteins that might accumulate in the ER and trigger ER stress during hibernation period. This will open a new area of study to explore in ground squirrel organs and new target genes to analyze that will provide a clear understanding of how mammalian hibernators survive and reverse the physiological state of torpor.

The third hypothesis on muscle disuse atrophy was supported by the inactivation of FoxOla via phosphorylation and low levels of proteins involved in the induction of muscle atrophy, in ground squirrel heart and muscle. Heart and skeletal muscle showed substantial differences in their patterns of expression of these proteins. This might be due 
to the fact that heart has to keep working while the animal is in torpor and even has to exert more force because of the low flow rates and increased blood viscosity at cold body temperatures. By contrast, skeletal muscle is largely inactive and appears to undergo some atrophy. AKT signaling to FOXO may represent only one part of the signaling involved in the atrophy process, and much remains to be learned. Research into the molecular and cellular mechanisms controlling muscle wasting is ongoing and important progress continues to be made. Future studies that investigate signaling pathways involving transcription factors that could regulate the balance between muscle protein synthesis and degradation during torpor should provide important insights to better understand the molecular pathways leading to muscle loss. Moreover, increased understanding of the molecular regulation of muscle wasting in hibernating mammals may help in the future to develop novel treatment strategies in patients with muscle wasting diseases. The last hypothesis dealt with the probability that proteins involved in anti-apoptosis defense were enhanced in hibernation. Bcl-XL, Bcl-2 and phospho-BAD were all shown to increase in heart of hibernating ground squirrels and, therefore, probably play important protective roles against oxidative stress and ROS generated during hibernation. However, in skeletal muscle, even though Bcl-XL was increased, Bcl2 and all the phosphorylated forms of BAD were unchanged or strongly decreased. It would then be interesting to test the responses of other related proteins of anti-apoptotic defense to determine if the extrinsic pathway (involving inhibitors of caspases) of apoptosis is stimulated in the tissues of hibernating ground squirrels and whether antiapoptotic proteins such as XIAP and cIAP are elevated to supplement the anti-apoptotic response that occurs via the intrinsic pathway. 
In summary, this thesis examined differential expression of genes and transcription factors in selected tissues of hibernating thirteen-lined ground squirrels $(S$. tridecemlineatus), by the use of several techniques. I looked at aspects of metabolic regulation in hibernation by investigating transcriptional, translational and posttranslational responses of genes or proteins. I demonstrated that selected genes and transcription factors are differentially expressed, in an organ-specific manner during hibernation. Transcription factors activation has just begun to be investigated in hibernators and my thesis examined ATF4 (CREB2) and FoxO1a transcription factors that were shown to play key roles by regulating gene expression in torpid ground squirrels. 
Fig. 6.1: Analysis of grp78 expression in brown adipose tissue and brain of the little brown bat M. lucifugus. (A) Histograms showing the relative content of GRP78 protein in the two tissues. (B) Histograms showing the mean ratio of grp78 mRNA levels (relative to $\alpha$-tubulin) in the two organs. Data are means \pm S.E.M.. * - Values for the hibernating condition are significantly different from the corresponding euthermic values using the Student's $t$-test, $\mathrm{P}<0.05, \mathrm{n}=3$ independent trials with both hibernating and euthermic samples. Ratios were calculated as previously described in Chapter 2. 
Fig. 6.1

A. M. lucifugus GRP78 protein

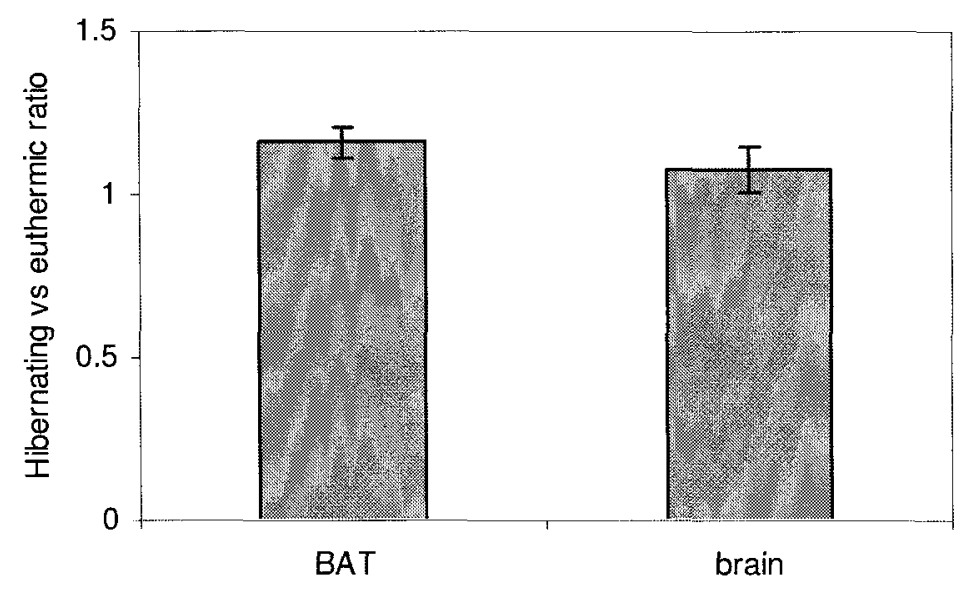

B. M. lucifugus grp78 mRNA

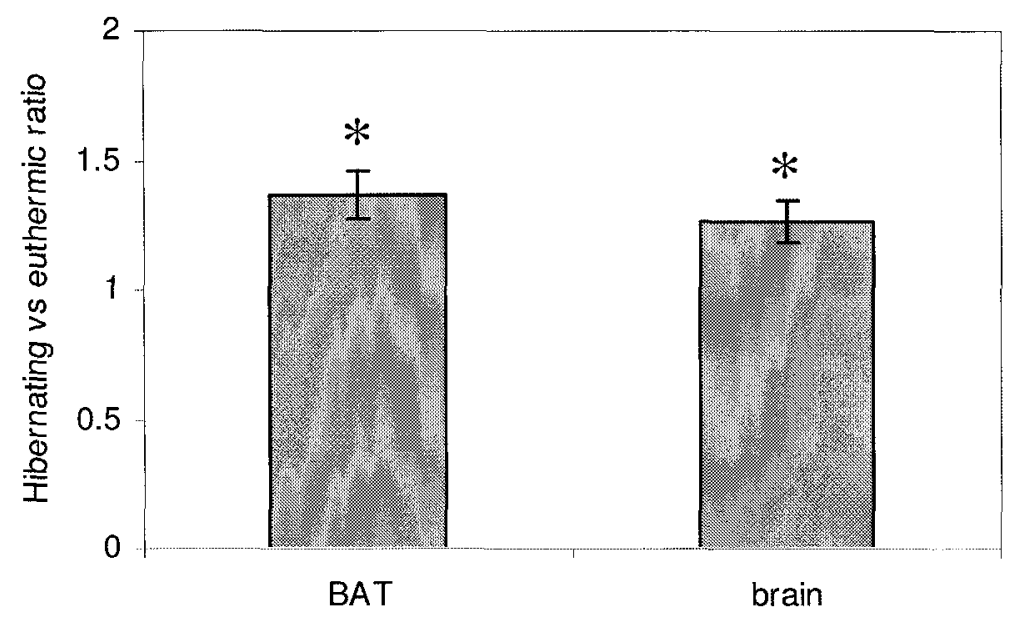


Fig. 6.2: A model for FoxO activation through the AKT signaling network during atrophy and hypertrophy. Phosphorylation of AKT by anabolic agents such as the insulin growth factor-1 leads to AKT activation and results in the activation of protein synthesis machinery via mTOR and downstream genes. Dephosphorylation of AKT by catabolic agents such as glucocorticoids results in the inactivation of AKT and dephosphorylation of FoxO proteins; this is followed by the translocation of FoxO into the nucleus where it regulates atrogenes (MAFbx, MuRf-1 and other atrophy-associated genes). Taken from Nader (2005). 
Fig. 6.2

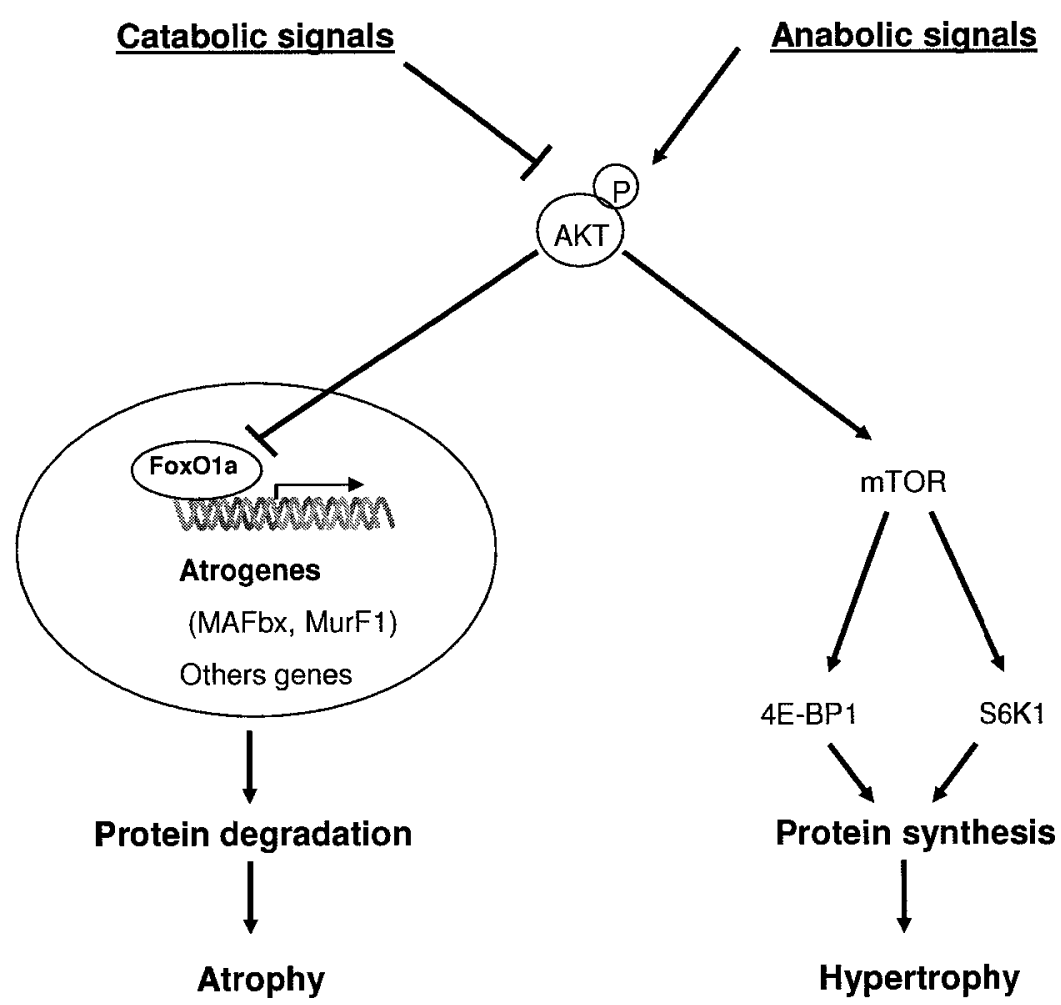




\section{Publication List}




\section{Research article in press}

Mamady, H. and Storey, K.B. 2006. Up-regulation of the endoplasmic reticulum molecular chaperone GRP78 during hibernation in thirteen-lined ground squirrels. Mol. Cell. Biochem. In press; Epub June 20, 2006.

\section{Research Submitted}

Mamady, H. and Storey, K. B. 2006. Expression of the activating transcription factor ATF4, ATF6 and downstream genes in the hibernating ground squirrels $S$. tridecemlineatus. Arch. Biochem. Biophys. Submitted

\section{Research Articles in Preparation}

Mamady, H. and Storey, K. B. Analysis of anti-apoptotic genes expression in tissues of euthermic versus hibernating thirteen-lined ground squirrels, S. tridecemlineatus. S. tridecemlineatus. In progress.

Mamady, H. and Storey, K. B. Hibernation and molecular responses to muscle disuse in S. tridecemlineatus. In progress.

Mamady, H., Abd El Halim, T. and Storey, K. B. Antioxidant defense: involvement of FoxO3a in the heart of hibernating thirteen-lined ground squirrel.

Mamady, H. and Storey, K. B. ER stress and unfolded protein response in hibernating bats, Myotis lucifugus.

Mamady, H. and Storey, K. B. Anti-apoptotic defense in tissues of Myotis lucifugus during hibernation. 


\section{GenBank Submissions}

Spermophilus tridecemlineatus glucose regulated protein $78 \mathrm{kDa}$ (GRP78) mRNA, complete cds. DQ166628.

Spermophilus tridecemlineatus activating transcription factor 4 (ATF4) mRNA, partial cds including 3'end. DQ324000.

Spermophilus tridecemlineatus Bcl- XL mRNA, partial cds. DQ503423.

\section{Communications at Scientific Meetings}

Hapsatou, M. and Storey, K.B. Expression of the activating transcription factor 4, ATF4, and its downstream genes in hibernating ground squirrels. CRYO-2005, $42^{\text {nd }}$ Annual Meeting of the Society for Cryobiology, Minneapolis, MN, July 24-27, 2005.

Hapsatou, M. and Storey, K.B. Title as above. $48^{\text {th }}$ annual meeting, Canadian Federation of Biological Societies, Guelph, ON, June 21-24, 2005.

Hapsatou, M. and Storey, K.B. ATF4 and unfolded protein response in hibernating ground squirrels. Oral Presentation. $44^{\text {th }}$ annual meeting, Canadian Society of Zoologists, Kingston, ON, May 10-14, 2005.

Hapsatou, M. and Storey, K.B. Expression of the activating transcription factor, ATF4, and its downstream genes during hibernation in ground squirrels. Ottawa Life Sciences Council: BioNorth 2004, Ottawa, November 29-December 1, 2004.

Hapsatou, M. and Storey, K.B. Title as above. $7^{\text {th }}$ Annual Chemistry \& Biochemistry Graduate Research Conference, Concordia University, Montreal, November 19$20,2004$. 
Hapsatou, M. and Storey, K.B. Induction of the endoplasmic reticulum molecular chaperone GRP78 in hibernating mammals. $3^{\text {rd }}$ International Conference of Comparative Physiology and Biochemistry in Africa, Ithala Game Reserve, KwaZulu-Natal, South Africa, August 7-13, 2004.

Hapsatou, M. and Storey, K.B. Title as above. Life in the Cold, $12^{\text {th }}$ International Symposium, Vancouver, BC - Seward, Alaska. July 25-August 1, 2004.

Hapsatou, M. and Storey, K.B. Title as above. Ottawa-Carleton Institute of Biology (OCIB) Conference. Ottawa, April 2004.

Hapsatou, M. and Storey, K.B. Title as above. Ottawa Life Sciences Council: BioNorth 2003, Ottawa, November 17-19, 2003.

Hapsatou, M. and Storey, K.B. Title as above. $6^{\text {th }}$ Annual Chemistry \& Biochemistry Graduate Research Conference, Concordia University, Montreal, November 14$15,2003$.

Hapsatou, M. and Storey, K.B. Glucose regulated proteins in hibernating mammals. Canadian Federation of Biological Societies, Ottawa, June 11 - 15, 2003. 


\section{REFERENCES}


Alexandre, S., Nakaki, T., Vanhamme, L. and Lee, A.S. 1991. A binding site for the cyclic adenosine 3',5'-monophosphate-response element binding protein as a regulatory element in the grp78 promoter. Mol. Endocrinol. 5: 1862-1878. .

Anderson, M.J., Viars, C.S., Czekay, S., Cavanee, W.K. and Arden, K.C. 1998. Cloning and characterization of three human Forkhead genes that comprise an FKHR-like gene subfamily. Genomics 47: 187-199.

Andrews, M.T., Squire, T.L., Bowen, C.M., and Rollins, M.B. 1998. Low-temperature carbon utilization is regulated by novel gene activity in the heart of a hibernating mammal. Proc. Natl. Acad. Sci. USA 95: 8392-8397.

Barja de Quiroga, G. 1992. Brown fat thermogenesis and exercise: two examples of physiological oxidative stress? Free Radic. Biol. Med. 13: 325-340.

Barthel, A., Schmoll, D. and Unterman, T.G. 2005. FoxO proteins in insulin action and metabolism. Trends Endocrinol. Metab. 16: 183-189.

Birkenkamp, K.U. and Coffer, P.J. 2003. Regulation of cell survival and proliferation by the FOXO (Forkhead box, class $\mathrm{O}$ ) subfamily of transcription factors. Biochem. Soc. Trans. 31: 292-297.

Blais, J.D., Philipenko, V., Bi, M., Harding, H.P., Ron, D., Koumenis, C., Wouters, B.G. and Bell J.C. 2004. Activating transcription factor 4 is translationally regulated by hypoxic stress. Mol. Cell. Biol. 24: 7469-7482.

Booth, F.W. and Thomason, D.B. 1991. Molecular and cellular adaptation of muscle in response to exercise: perspectives of various models. Physiol. Rev. 71: 541-585.

Boyer, B.B. and Barnes, B.M. 1999. Molecular and metabolic aspects of mammalian hibernation. Bioscience 49: 713-724. 
Brunet, A., Bonni, A., Zigmond, M.J., Lin, M.Z., Juo, P., Hu, L.S., Anderson, M.J., Arden, K.C., Blenis, J. and Greenberg, M.E. 1999. Akt promotes cell survival by phosphorylating and inhibiting a forkhead transcription factor. Cell 96: 857868.

Buck, C.L. and Barnes, B.M. 2000. Effects of ambient temperature on metabolic rate, respiratory quotient, and torpor in an arctic hibernator. Am. J. Physiol. 279: R255R262.

Buck, M.J., Squire, T.L. and Andrews, M.T. 2002. Coordinate expression of the PDK4 gene: a means of regulating fuel selection in a hibernating mammal. Physiol. Genomics 8: 5-13.

Burgering, B.M. and Kops, G.J. 2002. Cell cycle and death control: long live Forkheads. Trends Biochem. Sci. 27: 352-360.

Buzadzic, B., Spasic, M., Saicic, Z.S., Radojicic, R., Petrovic, V.M. and Halliwell, B. 1990. Antioxidant defenses in the ground squirrel Citellus citellus. 2. The effect of hibernation. Free Radic. Biol. Med. 9: 407-413

Cai, D., McCarron, R.M. and Hallenbeck, J. 2004a. Cloning and characterization of forkhead transcription factor gene, FoxOla, from thirteen-lined ground squirrel. Gene 343: 203-209.

Cai, D., McCarron, R.M., Yu, E.Z., Li, Y. and Hallenbeck, J.M. 2004b. Akt phosphorylation and kinase activity are down-regulated during hibernation in the thirteen-lined ground squirrel. Brain Res. 1014: 14-21.

Calvert, M.E., Digilio, L.C., Herr, J.C. and Coonrod, S.A. 2003. Oolemmal proteomics - identification of highly abundant heat shock proteins and molecular 
chaperones in the mature mouse egg and their localization on the plasma membrane. Reprod. Biol. Endocrinol. 1: 27-37.

Cao, P.R., Kim, H.J. and Lecker, S.H. 2004. Ubiquitin-protein ligases in muscle wasting. Int. J. Biochem. Cell Biol. 37: 2088-2097.

Carey, H.V., Frank, C.L. and Seifert, J.P. 2000. Hibernation induces oxidative stress and activation of NF-kappaB in ground squirrel intestine. J. Comp. Physiol. B 170: $551-559$.

Carey, H.V., Andrews, T. and Martin, S.L. 2003a. Mammalian hibernation: cellular and molecular responses to depressed metabolism and low temperature. Physiol. Rev. 83: $1153-1181$

Carey, H.V., Rhoads, C.A. and Aw, T.Y. 2003b. Hibernation induces glutathione redox imbalance in ground squirrel intestine. J. Comp. Physiol. B 173: 269-276.

Ciriolo, M.R. 2005. Redox control of apoptosis. Antioxid. Redox Signal. 7: 432-435.

Chang, B.S., Minn, A.J., Muchmore, S.W., Fesik, S.W. and Thompson, C.B. 1997. Identification of novel regulatory domain in Bcl-xL and Bcl-2. EMBO J. 16: 968977.

Chen, Y., Matsushita, M., Nairn, A.C., Damuni, Z., Cai, D., Frerichs, K.U. and Hallenbeck, J.M. 2001. Mechanisms for increased levels of phosphorylation of elongation factor-2 during hibernation in ground squirrels. Biochemistry 40: $11565-11570$.

Cleary, E.C. and Craven, S.R. 1994. Thirteen-lined ground squirrels. In Prevention and Control of Wildlife Damage. Ed. S.E. Hygnstrom, R.M. Timm and G.E. Larson. Institute of Agriculture and Natural Resources, University of Nebraska, Lincoln. 
Clemens, M.J. 2001. Initiation factor eIF2 $\alpha$ phosphorylation in stress responses and apoptosis. Prog. Mol. Subcell. Biol. 27: 57-89.

Cullinan, S.B. and Diehl, J.A. 2006. Coordination of ER and oxidative stress signaling: the PERK/Nrf2 signaling pathway. Int. J. Biochem. Cell Biol. 38: 317-332.

Dausmann, K.H., Ganzhorn, J.U. and Heldmaier, G. 2000. Body temperature and metabolic rate of a hibernating primate in Madagascar: preliminary results from a field study. In: Life in the Cold. Ed. G. Heldmaier and M. Klingenspor. Springer, Berlin. pp. 41-47.

Dignam, J.D., Lebovitz, R.M. and Roeder, R.G. 1983. Accurate transcription initiation by RNA polymerase II in a soluble extract from isolated mammalian nuclei. Nuc. Acids Res. 11: 1475-1489.

Drew, K.L., Toien, O., Rivera, P.M., Smith, M.A., Perry, G. and Rice, M.E. 2002. Role of the antioxidant ascorbate in hibernation and warming from hibernation. Comp. Biochem. Physiol. C 133: 483-492.

Eddy, S.F. and Storey, K.B. 2003. Differential expression of Akt, PPAR- $\gamma$ and PGC-1 during hibernation in bats. Biochem. Cell Biol. 81: 269-274.

Eddy, S.F. and Storey, K.B. 2004. Up-regulation of fatty acid-binding proteins during hibernation in the little brown bat, Myotis lucifugus. Biochim. Biophys. Acta 1676: $63-70$.

Eddy, S.F., Morin, P. and Storey, K.B. 2005a. Cloning and expression of PPAR $\gamma$ and PGC-1 $\alpha$ from the hibernating ground squirrel, Spermophilus tridecemlineatus. Mol. Cell. Biochem. 269: 175-182.

Eddy, S.F., McNally, J.D. and Storey, K.B. 2005b. Up-regulation of a thioredoxin 
peroxidase-like protein, proliferation associated gene, in hibernating bats. Arch. Biochem. Biophys. 435: 101-111.

El Ouezzani, S., Lafon, P., Tramu, G. and Magoul, R. 2001. Neuropeptide Y gene expression in the jerboa arcuate nucleus: modulation by food deprivation and relationship with hibernation. Neurosci. Lett. 305: 127-130.

Fahlman, A., Storey, J.M. and Storey, K.B. 2000. Gene up-regulation in the heart during mammalian hibernation. Cryobiology 40: 332-342.

Fernandez, J., Yaman, I., Sarnow, P., Snider, M.D. and Hatzoglou, M. 2002. Regulation of internal ribosomal entry site-mediated translation by phosphorylation of the translation initiation factor eIF2alpha. J. Biol. Chem. 277: 19198-19205.

Fleck, C.C. and Carey, H.V. 2005. Modulation of apoptotic pathways in the intestinal mucosa during hibernation. Am. J. Physiol. 289: R586-R595.

French, A.R. 1988. Patterns of mammalian hibernation. Am. Sci. 76: 569-575.

Frerichs, K.U., Kennedy, C., Sokoloff, L. and Hallenbeck, J.M. 1994. Local cerebral blood flow during hibernation, a model of natural tolerance to "cerebral ischemia" J. Cereb. Blood Flow Metab. 14: 193-205.

Frerichs, K. U., Dienel, G., Sokoloff, L. and Hallenbeck, J.M. 1995. Rates of glucose utilization in brain of active and hibernating ground squirrels. Am. J. Physiol. 268: R445-R453.

Frerichs, K.U., Smith, C.B., Brenner, M., DeGracia, D.J., Krause, G.S., Marrone, L., Dever, T.E. and Hallenbeck, J.M. 1998. Suppression of protein synthesis in brain during hibernation involves inhibition of protein initiation and elongation. 
Proc. Natl. Acad. Sci. USA 95: 14511-14516.

Furuyama, T., Nakazawa, T., Nakano, I. and Mori, N. 2000. Identification of the differential distribution patterns of mRNAs and consensus binding sequences for mouse DAF-16 homologues. Biochem. J. 349: 629-634.

Geiser, F. 1988. Reduction of metabolism during hibernation and daily torpor in mammals and birds: temperature effect or physiological inhibition? J. Comp. Physiol. B 158: 25-38.

Geiser, F. and Kenagy, G.J. 1988. Duration of torpor bouts in relation to temperature and energy metabolism in hibernating ground squirrels. Physiol. Zool. 61: 442449.

Geiser, F. and Ruf, T. 1995. Hibernation versus daily torpor in mammals and birds: physiological variables and classification of torpor patterns. Physiol. Zool. 68: 935-966.

Gething, M.-J. 1999. Role and regulation of the ER chaperone BiP. Cell. Devel. Biol. 10: $465-472$.

Glass, D.J. 2005. Skeletal muscle hypertrophy and atrophy signaling pathways. Int. J. Biochem. Cell Biol. 37: 1974-1984.

Gross, A., Mcdonnell, J.M. and Korsmeyer, S.J. 1999. Bcl-2 family members and the mitochondria in apoptosis. Gene Devel. 13: 1899-1911.

Harding, H.P., Novoa, I., Zhang, Y., H., Zeng, H., Wek, R. Schapira, M. and Ron, D. 2000. Regulated translation initiation controls stress-induced gene expression in mammalian cells. Mol. Cell. 6: 1099-1108.

Hittel, D. and Storey, K.B. 2001. Differential expression of adipose- and heart-type fatty 
acid binding proteins in hibernating ground squirrels. Biochem. Biophys. Acta 1522: 238-243.

Hittel, D. and Storey, K.B. 2002. The translation state of differentially expressed mRNAs in the hibernating 13-lined ground squirrel (Spermophilus tridecemlineatus). Arch. Biochem. Biophys. 401: 244-254.

Huang, D.C., Adams, J.M. and Cory, S. 1998. The conserved N-terminal BH4 domain of $\mathrm{Bcl}-2$ homologues is essential for inhibition of apoptosis and interaction with CED-4. EMBO J. 17: 1029-1039.

Humphries, M.M., Thomas, D.W. and Kramer, D.L. 2003. The role of energy availability in mammalian hibernation: a cost-benefit approach. Physiol. Biochem. Zool. 76: 165-179

\section{Hunter, R.B., Stevenson, E.J., Koncarevic, A., Mitchell-Felton, H., Essig, D.A. and} Kandarian, S.C. 2002. Activation of an alternative NF- $\mathrm{BB}$ pathway in skeletal muscle during disuse atrophy. FASEB J. 16: 529-538.

Hunter, R.B. and Kandarian, S.C. 2004. Disruption of either the Nfkb1 or the Bcl3 gene inhibits skeletal muscle atrophy. J. Clin. Investig. 114: 1504-1511.

Jackman, R.W. and Kandarian, S.C. 2004. The molecular basis of skeletal muscle atrophy. Am. J. Physiol. Cell. Physiol. 287: C834-843.

Jackson, P.K., Eldridge, A.G., Freed, E., Furstenthal, L., Hsu, J.Y., Kaiser, B.K. and Reimann, J.D.R. 2000. The lore of the RINGs: substrate recognition and catalysis by ubiquitin ligases. Trends Cell. Biol. 10: 429-439.

Janumyan, Y.M., Sansam, C.G., Chattopadhyay, A., Cheng, N., Soucie, E.L., Penn, L.Z., Andrews, D., Knudson, C.M. and Yang, E. 2003. Bcl-XL/Bcl-2 
coordinately regulates apoptosis, cell cycle arrest and cell cycle entry. EMBO J. 22: $5459-5470$.

Jiang, H.-Y. and Wek, R.C. 2005. Phosphorylation of the $\alpha$-subunit of the eukaryotic initiation factor-2 (elf $2 \alpha)$ reduces protein synthesis and enhances apoptosis in response to proteasome inhibition. J. Biol. Chem. 260: 14189-14202.

Kamei, Y., Miura, S., Suzuki, M., Kai, Y., Mizukami, J., Taniguchi, T., Mochida, K., Hata, T., Matsuda, Aburatani, H., Nishino, I. and Ezaki, O. 2004. Skeletal muscle FOXO1 (FKHR) transgenic mice have less skeletal muscle mass, downregulated type I (slow twitch/red muscle) fiber genes, and impaired glycemic control. J. Biol. Chem. 279: 41114-41123.

Kilduff, T.S., Miller, J.D., Radeke, C.M., Sharp, F.R. and Heller, H.C. $1990 .{ }^{14}$ C-2deoxyglucose uptake in the ground squirrel brain during entrance to and arousal from hibernation. J. Neurosci. 10: 2463-2475.

Knight, J.E., Narus, E.N., Martin, S.L., Jacobson, A., Barnes, B.M. and Boyer, B.B. 2000. mRNA stability and polysome loss in hibernating Arctic ground squirrels (Spermophilus parryii). Mol Cell Biol. 20: 6374-6379.

Kozak, M. 2002. Pushing the limits of the scanning mechanism for initiation of translation. Gene 299: 1-34.

Kops, G.J. and Burgering, B.M. 2000. Forkhead transcription factors are targets of signaling by the proto-oncogene PKB (C-AKT). J. Anat. 197: 571-574.

Koumenis, C., Denko, N.C., Koritzinski, M., Rastani, S., Diehl, A., Sonenberg, N., Koromilas, A. and Wouters, B.G. 2002. Regulation of protein synthesis by hypoxia via activation of the endoplasmic reticulum kinase PERK and 
phosphorylation of the translation initiation factor eIF2 $\alpha$. Mol. Cell. Biol. 22: $7405-7416$.

Kozutsumi, Y., Segal, M., Normington, K., Gething, M.-J. and Sambrook, J. 1988. The presence of malfolded proteins in the endoplasmic reticulum signals the induction of glucose-regulated proteins. Nature 332: 462-464.

Kurtz, C.C., Lindell, S.L., Mangino, M.J., Carey, H.V. 2006. Hibernation confers resistance to intestinal ischemia-reperfusion injury. Am. J. Physiol. Gastrointest. Liver Physiol. Jun 1- [Epub ahead of print].

Lee, A.S. 2001. The glucose-regulated proteins: stress induction and clinical applications. Trends Biochem. Sci. 26: 504-510.

Lee, A.S. 2005. The ER chaperone and signaling regulator GRP78/BiP as a monitor of endoplasmic reticulum stress. Methods 35: 373-381.

Lee, M., Choi, I. and Park, K. 2002. Activation of stress signaling molecules in bat brain during arousal from hibernation. J. Neurochem. 82: 867-873.

Luo, S., Baumeinster, P., Yang, S., Abcouwer, S.F. and Lee, A.S. 2003. Induction of GRP78/BiP by translational block. Activation of the Grp78 promoter by ATF4 through an upstream ATF/CRE site independent of the endoplasmic reticulum stress elements. J. Biol. Chem. 378: 37375-37385.

Lyman, C.P., Willis, J.S., Malan, A. and Wang L.C.H. 1982. Hibernation and torpor in mammals and birds. Academic Press, New York.

Ma, Y. and Hendershot, L.M. 2003. Delineation of a negative feedback regulatory loop that controls protein translation during endoplasmic reticulum stress. J. Biol. Chem. 278: 34864-34873. 
MacDonald, J.A. and Storey, K.B. 2005. Mitogen-activated protein kinases and selected downstream targets display organ-specific responses in the hibernating ground squirrel. Int. J. Biochem. Cell Biol. 37: 679-691.

McArthur, M.D. and Milsom, W.K. 1991. Changes in ventilation and respiratory sensitivity associated with hibernation in Columbian (Spermophilus columbianus) and golden-mantled (Spermophilis lateralis) ground squirrels. Physiol. Zool. 64: 940-959.

McCullough, K.D., Martindale, J.L., Klotz, L.O., Aw, T.Y. and Holbrook, N.J. 2001. Gadd153 sensitizes cells to endoplasmic reticulum stress by down-regulating Bcl2 and perturbing the cellular redox state. Mol. Cell. Biol. 21: 1249-1259.

Mamady, H. and Storey, K.B. 2006. Up-regulation of the endoplasmic reticulum molecular chaperone GRP78 during hibernation in thirteen-lined ground squirrels. Mol. Cell. Biochem. in press; Epub June 20, 2006.

Mastrangelo, A.J., Hardwick, J.M., Zou, S., Betenbaugh, M.J. 2000. Overexpression of Bcl-2 families enhances survival of mammalian cells in response to various culture insults. Biotechnol. Bioeng. 67: 555-564.

Mata, J., Marguerat, S. and Bähler, J. 2005. Post-transcriptional control of gene expression: a genome-wide perspective. Trends Biochem. Sci. 30: 506-514.

Morin, P.J. and Storey, K.B. 2005. Cloning and expression of hypoxia-inducible factor $1 \alpha$ from the hibernating ground squirrel, Spermophilus tridecemlineatus. Biochem. Biophys. Acta 1729: 32-40.

Milsom, W.K., Zimmer, M.B., and Harris, M.B. 1999. Regulation of cardiac rhythm in hibernating mammals. Comp. Biochem. Physiol. A 124: 383-391. 
Munro, S. and Pelham, H.R.B. 1987. A C-terminal signal prevents secretion of luminal ER proteins. Cell 48: 899-907.

Nader, G.A. 2005. Molecular determinants of skeletal muscle mass: getting the "AKT" together. Int. J. Biochem. Cell Biol. 37: 1985-1996.

Neuweiler, G. 2000. The biology of bats. New York: Oxford University Press.

Noda, T., Iwakiri, R., Fujimoto, K., Matsuo, S. and Aw, T.Y. 1998. Programmed cell death induced by ischemia-reperfusion in rat intestinal mucosa. Am. J. Physiol. 274: G270-G276.

O’Hara, B., Watson, F.O., Srere, H.K., Kumar, H., Wiler, S.W., Welch, S.K., Bitting, L., Heller, H.C. and Kilduff, T.S. 1999. Gene expression in the brain across the hibernation cycle. J. Neurosci. 19: 3781-3790.

Okada, T., Yoshida, H., Akazawa, R., Negishi, M. and Mori, K. 2002. Distinct roles of activating transcription factor 6 (ATF6) and double-stranded RNA-activated protein kinase-like endoplasmic reticulum kinase (PERK) in transcription during the mammalian unfolded protein response. Biochem. J. 366: 585-594.

Osborne, P.G. and Hashimoto, M. 2006. Brain antioxidant levels in hamsters during hibernation, arousal and cenothermia. Behav. Brain. Res. 168: 208-214.

Patil, C. and Walter, P. 2001. Intracellular signalling from the endoplasmic reticulum to the nucleus: the unfolded protein response in yeast and mammals. Curr. Opin. Cell. Biol. 13: 349-355.

Pelham, H.R.B. 1990. The retention signal for soluble proteins of the endoplasmic reticulum. Trends Biochem. Sci. 15: 483-486.

Ramachandra, K.R., Changhui, M., Baumeister, P., Austin, R.C., Kaufman, R.J. and 
Lee, A.S. 2003. Endoplasmic reticulum chaperone protein GRP78 protects cells from apoptosis induced by topoisomerase inhibitors: role of ATP binding site in suppression of caspase-7 activation. J. Biol. Chem. 278: 20915-20924.

Rena, G., Guo, S., Cichy, S.C., Unterman, T.G. and Cohen, P. 1999. Phosphorylation of the transcription factor forkhead family member FKHR by protein kinase B. J. Biol. Chem. 274: 17179-17183.

Rena, G.., Prescott, A.R., Guo, S., Cohen, P. and Unterman, T.G. 2001. Roles of the forkhead in rhabdomyosarcoma (FKHR) phosphorylation sites in regulating 14-33 binding, transactivation and nuclear targeting. Biochem. J. 354: 605-612.

Rena, G., Woods, Y.L., Prescott, A.R., Peggie, M., Unterman, T.G., Williams, M.P. and Cohen, P. 2002. Two novel phosphorylation sites on FKHR that are critical for its nuclear exclusion. EMBO J. 21: 2263-2271.

Rourke, B.C., Yokoyama, Y., Milsom, W.K. and Caiozzo, V.J. 2004. Myosin isoform expression and MAFbx mRNA levels in hibernating golden-mantled ground squirrels (Spermophilus lateralis). Physiol. Biochem. Zool. 77: 582-593.

Roy, B. and Lee, A.S. 1999. The mammalian endoplasmic reticulum stress response element consists of an evolutionarily conserved tripartite structure and interacts with a novel stress-inducible complex. Nuc. Acids Res. 27: 1437-1443.

Rutkowski, D.T. and Kaufman, R.J. 2004. A trip to the ER: coping with stress. Trends Cell. Biol. 14: 20-28.

Sandri, M., Sandri, C., Gilbert, A., Skruk, C., Calabria, E., Picard, A., Walsh, K., Schiaffino, S., Lecker, S.H. and Goldberg, A.L. 2004. Foxo transcription factors induce the atrophy-related ubiquitin ligase atrogin- 1 and cause skeletal muscle 
atrophy. Cell 117: 399-412.

Schmidt, M., Fernandez de Mattos, S., Van der Horst, A., Klompmaker, R., Kops, G.J., Lam, E.W., Burgering B.M. and Medema, R.H. 2002. Cell cycle inhibition by FoxO forkhead transcription factors involves down-regulation of cyclin D. Mol. Cell. Biol. 22: 7842-7852.

Schröder, M. and Kaufman, R.J. 2005. The mammalian unfolded protein response. Annu. Rev. Biochem. 74: 739-789, 2005.

Shen, J. and Prywes, R. 2005. ER stress signaling by regulated proteolysis of ATF6. Methods 35: 382-9.

Speakman, J. R. and D. W. Thomas. 2003. Physiological ecology and energetics of bats. In Bat Ecology. Ed. T. H. Kunz and M. B. Fenton. University of Chicago Press, Chicago. pp 430-490.

Stitt, T.N., Drujan, D., Clarke, B.A., Panaro, F.J., Timofeyva, Y., Kline, W.O., Gonzalez, M., Yancopoulos, G.D. and Glass, D.J. 2004. The IGF-1/PI3K/Akt pathway prevents expression of muscle atrophy-induced ubiquitin ligases by inhibiting FOXO transcription factors. Mol. Cell. 14: 395-403.

Storey, K.B. 2003. Mammalian hibernation: transcriptional and translational controls. Adv. Exp. Med. Biol. 543: 21-38.

Storey, K.B. 2004. Cold, ischemic organ preservation: lessons from natural systems. J. Invest. Med. 52: 315-322.

Storey, K.B. and Storey J.M. 2004. Metabolic rate depression in animals: transcriptional and translational controls. Biol. Rev. 79: 207-233.

Strasser, A., O'Connor, L. and Dixit, V.M. 2000. Apoptosis signaling. Annu. Rev. 
Biochem. 69: 217-245.

Tan, Y.T., Demeter, M.R., Ruan, H. and Comb, M.J. 2000. BAD Ser-155 phosphorylation regulates $\mathrm{BAD} / \mathrm{Bcl}-\mathrm{XL}$ interaction and cell survival. J. Biol. Chem. 275: 25865-25869.

Thomas, D.W. and Cloutier D. 1992. Evaporative water loss by hibernating little brown bats, Myotis lucifugus. Physiol. Zool. 65:433-456.

Thomas, D.W. and Geiser, F. 1997. Periodic arousals in hibernating mammals: is evaporative water loss involed? Funct. Ecol. 11: 585-591.

Thomason, D.B. and Booth, F.W. 1990. Atrophy of the soleus muscle by hindlimb unweighting. J. Appl. Physiol. 68: 1-12.

Tran, H., Brunet, A., Griffith, E.C. and Greenberg, M.E. 2003. The many forks in FOXO's road. Sci. STKE 2003, 172: RE5.

Van Der Heide, L.P., Hoekman, M.F.M. and Smidt, M.P. 2004. The ins and outs of FoxO shuttling: mechanisms of FoxO translocation and transcriptional regulation. Biochem. J. 380: 297-309.

Vaux, D.L. and Korsmeyer, S.J. 1999. Cell death in development. Cell 96: 245-254.

Vaux, D.L. and Silke, J. 2005. IAPs, rings and ubiquitylation. Nature Rev. Mol. Cell. Biol. 6: 287-297.

Wang, L.C.H. 1989. Ecological, physiological and biochemical aspects of torpor in mammals and birds. In: Advances in Comparative and Environmental Physiology. Ed. L.C.H. Wang. Springer-Verlag. Berlin. Vol. 4, pp.361-401.

Wang, L.C.H. and Lee, T.F. 1996. Torpor and hibernation in mammals: metabolic, physiological and biochemical adaptations. In Handbook of Physiology: 
Environmental Physiology. Ed. M.J. Fregley and C.M. Blatteis. Oxford University Press, New York. Section 4, Vol. 1, pp.507-532.

Wang, X. Z., Lawson, B., Brewer, J. W., Zinszner, H., Sanjay, A., Mi, L. J., Boorstein, R., Kreibich, G., Hendershot, L. M., and Ron, D. 1996. Signals from the stressed endoplasmic reticulum induce C/EBP-homologous protein (CHOP/GADD153). Mol. Cell. Biol. 16: 4273-4280.

Wang, Y., Shen, J., Arenzana, N. Tirasophon, W., Kaufman, R.J. and Prywes, R. 2000. Activation of ATF6 and an ATF6 DNA binding site by the endoplasmic reticulum stress response. J. Biol. Chem. 275: 27013-27020.

Wickler, S.J., Hoyt, D.F. and Kott, K.S. 1991. Disuse atrophy in the hibernating golden-mantled ground squirrel, Spermophilus lateralis. Am. J. Physiol. 261: R1214-R1217.

Wooden, S.K. and Lee, A.S. 1992. Comparison of the genomic organizations of the rat grp78 and hsc73 gene and their evolutionary implications. DNA Seq. 3: 41-48.

Woods, Y.L., Rena, G., Morrice, N., Barthel, A., Beckec, W., Guo, S., Unterman, T.G. and Cohen, P. 2001. The kinase DYRK1A phosphorylates the transcription factor FKHR at Ser329 in vitro, a novel in vivo phosphorylation site. Biochem. J. 355: 597-607.

Yacoe, M.E. 1983. Protein metabolism in the pectoralis muscle and liver of hibernating bats, Eptesicus fuscus. J. Comp. Physiol. 152: 137-144.

Yang, E., Zha, J., Jockel, J., Boise, L.H., Thompson, C.B. and Korsmeyer, S.J. 1995. $\mathrm{Bad}$, a heterodimeric partner of $\mathrm{Bcl}-\mathrm{xL}$ and $\mathrm{Bcl}-2$, displaces $\mathrm{Bax}$ and promotes cell death. Cell 80: 285-291 
Yoshida, H. Okada, T., Haze, K., Yanagi, H., Yura, T., Negishi, M. And Mori, K. 2000. ATF6 activated by proteolysis binds in the presence of NF-Y (CBF) directly to the cis-acting element responsible for the mammalian unfolded protein response. Mol. Cell. Biol. 20: 6755-6767.

Zamzami, N., Brenner, C., Marzo, I., Susin, S.A. and Kroemer, G. 1998. Subcellular and submitochondrial mode of action of Bcl-2 like oncoproteins. Oncogene 16: 2265-2282.

Zatzman, M.L. 1984. Renal and cardiovascular effects of hibernation and hypothermia. Cryobiology 21: 593-614.

Zha, J., Harada, H., Yang, E., Jockel, J. and Korsmeyer, S.J. 1996. Serine phosphorylation of death agonist BAD in response to survival factor results in binding to 14-3-3 not Bcl-x (L). Cell 87: 619-628.

Zhegunov, G.F, Mikulinsky, Y.E, and Kudokotseva, E.V. 1988. Hyperactivation of protein synthesis in tissues of hibernating animals on arousal. Cryo-Lett 9: 236245.

Zhou, F., Smith, M.A., Castellani, R.J., Stimmelmayr, R., Perry, G., Drew, K.L., Zhu, X. 2001. Hibernation, a model of neuroprotection. Sci. World J. 1 (1 Suppl 3): 108. 